\title{
ORBITAL DETERMINATION FEASIBILITY OF LEO NANOSATELLITES USING SMALL APERTURE TELESCOPES
}

\author{
A Thesis \\ presented to \\ the Faculty of California Polytechnic State University, \\ San Luis Obispo
}

\author{
In Partial Fulfillment \\ of the Requirements for the Degree \\ Master of Science in Aerospace Engineering
}

by

Michael R. Strange

March 2017 
(C) 2017

Michael R. Strange

ALL RIGHTS RESERVED 


\section{COMMITTEE MEMBERSHIP}

TITLE: Orbital Determination Feasibility of LEO

Nanosatellites Using Small Aperture Telescopes

AUTHOR: Michael R. Strange

DATE SUBMITTED: March 2017

COMMITTEE CHAIR: Kira Abercromby, Ph.D.

Associate Professor of Aerospace Engineering

COMMITTEE MEMBER: Eric Mehiel, Ph.D.

Professor of Aerospace Engineering

COMMITTEE MEMBER: Vardha N. Bennert, Ph.D.

Assistant Professor of Physics

COMMITTEE MEMBER: David Mitchell, Ph.D.

Professor of Physics 


\begin{abstract}
Orbital Determination Feasibility of LEO Nanosatellites Using Small Aperture Telescopes

Michael R. Strange
\end{abstract}

This thesis is directed toward the feasibility of observing satellites on the nano scale and determining an accurate propagated orbit using a Meade LX600-ACF 14" diameter aperture telescope currently located on the California Polytechnic State University campus. The optical telescope is fitted with an f/6.3 focal reducer, SBIG ST-10XME CCD camera and Optec TCF-S Focuser. This instrumentation allowed for a 22' X 15' arcminute FOV in order to accurately image passing LEO satellites. Through the use of the Double-r and Gauss Initial Orbit Determination methods as well as Least Squared Differential Correction and Extended Kalman Filter Orbit Determination methods, an accurate predicted orbit can be determined.

These calculated values from observational data of satellites within the Globalstar system are compared against the most updated TLEs for each satellite at the time of observation. The determined differential errors from the well-defined TLEs acquired via online database were used to verify the feasibility of the accuracy which can be obtained from independent observations. Through minimization of error caused from imaging noise, pointing error, and timing error, the main determination of accurate orbital determination lies in the instrumentation mechanical capabilities itself. With the ability to acquire up to 7 individual satellite observations during a single transit, the use of both IOD and OD methods, and the recently acquired Cal Poly telescope with an increased 14" aperture, the feasibility of imaging and orbital determination of nanosatellites is greatly improved. 


\section{ACKNOWLEDGMENTS}

I would like to thank Dr. Kira Abercromby for being such a supportive and accommodating advisor throughout this entire process. Without the weekly meetings and shared distain for inaccurate space films, this would have been a much less enjoyable experience.

I would also like to thank my parents John and Wendi Strange and sister Kym for their unwavering persistence and optimism in completing my degree.

Lastly I would like to thank my college roommates Tim Fitzgerald, Michelle Haddock, and Chris Barlog for sharing in both the good times and the bad times of grad school. 


\section{TABLE OF CONTENTS}

\section{Page}

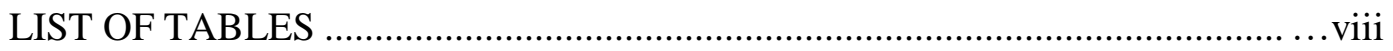

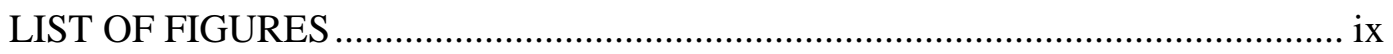

LIST OF ACRONYMS .............................................................................

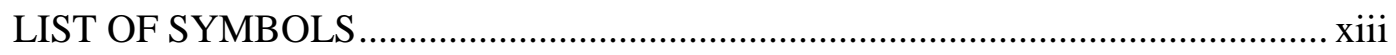

\section{CHAPTER}

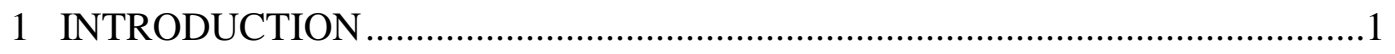

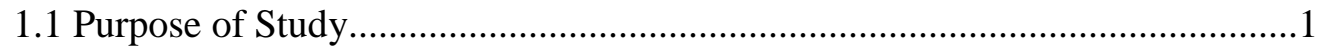

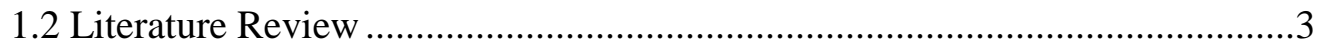

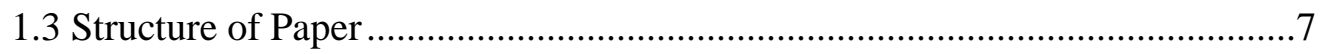

2 ASTRODYNAMICS BACKGROUND ..........................................................

2.1 Orbital Mechanics Review ........................................................................ 8

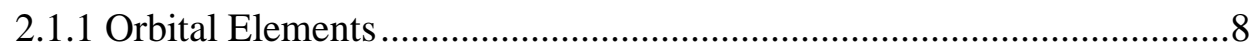

2.1.2 Two Line Element Sets ...................................................................11

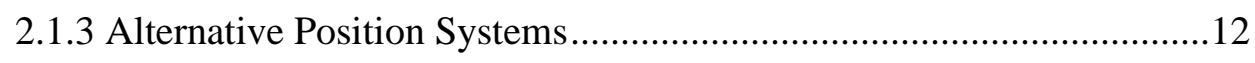

2.1.4 Celestial Coordinate Systems ........................................................12

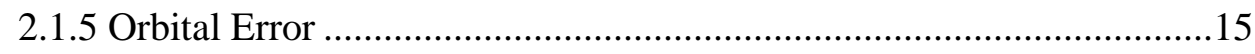

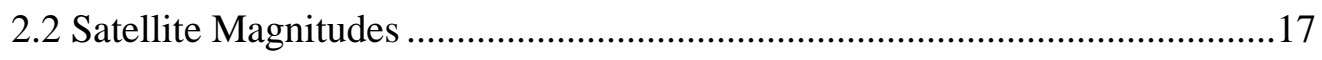

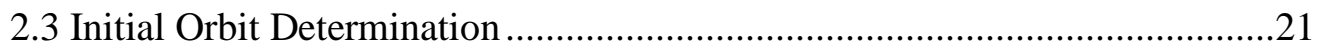

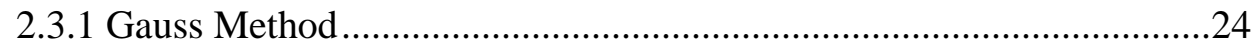

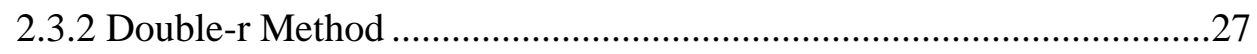

2.4 Orbit Determination and Estimation..........................................................30

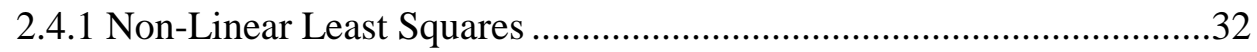

2.4.2 Extended Kalman Filtering ......................................................... 36

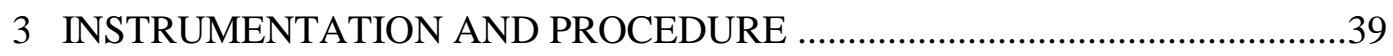

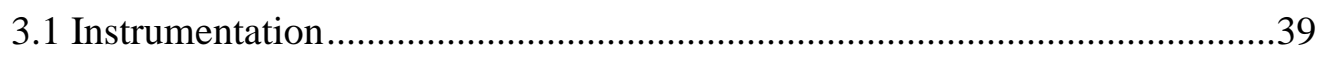

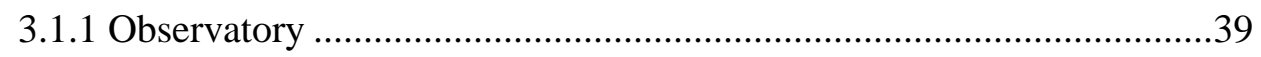

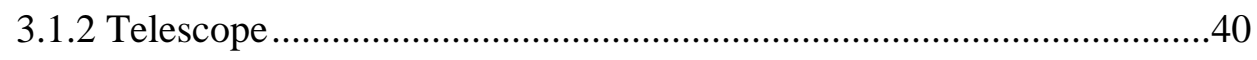




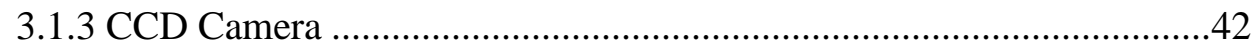

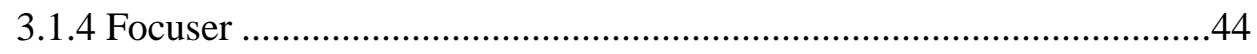

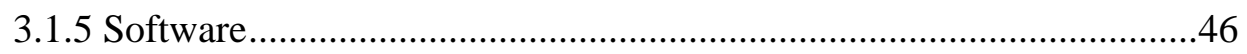

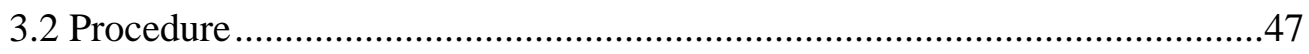

3.2.1 Procedural Overview .............................................................................4

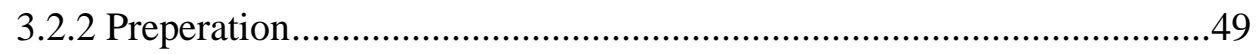

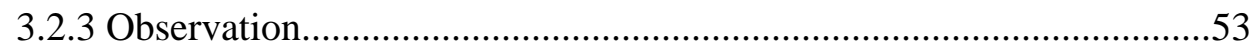

3.2.4 Photometric Reduction ....................................................................56

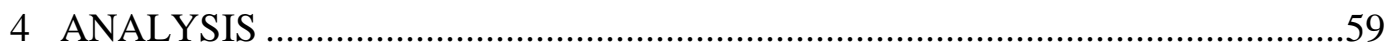

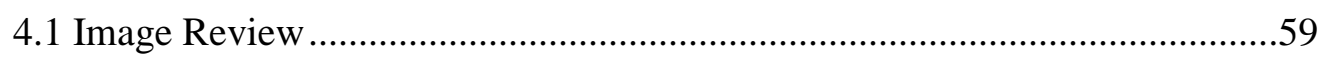

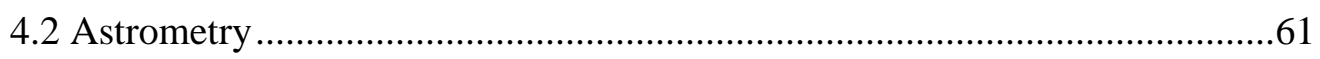

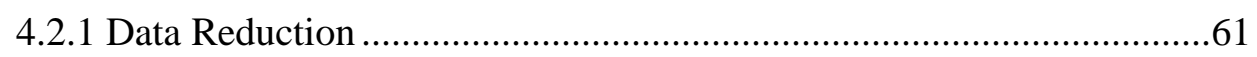

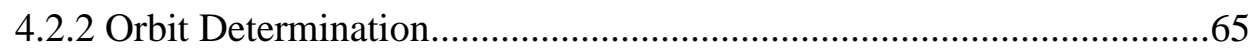

4.2.3 TLE Positional Error ...........................................................................66

4.2.4 Magnitude Feasibility ........................................................................68

5 RESULTS AND DISCUSSION .....................................................................

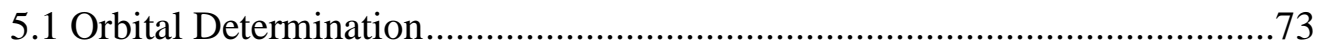

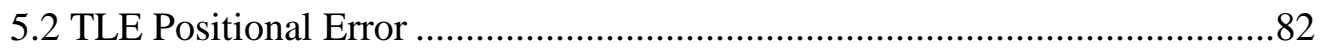

5.3 Imaging Methods ..........................................................................................

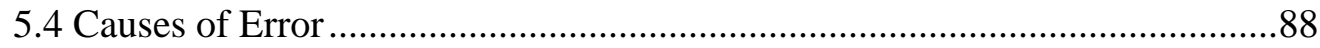

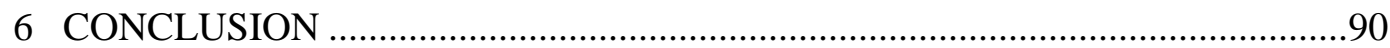

6.1 Recommendations for Future Work ........................................................91

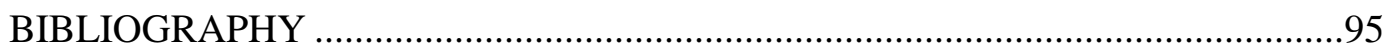

APPENDICES

A SELECTED IMAGES AND RAW DATA ………………………………....99

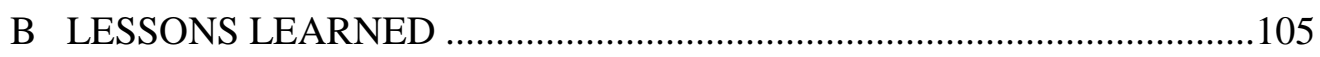

C SATELLITE TARGET ORBITAL DATA ……………………………........108

D OBSERVATION PROCEDURE ……………………...........................110

E PIXEL ERROR ANALYSIS …………………………………………....117 


\section{LIST OF TABLES}

Table

2.1 Apparent Magnitude Values ..............................................................21

3.1 Meade LX600-ACF 14” Telescope Specifications......................................41

3.2 SBIG ST-10XME CCD Camera Specifications ..........................................43

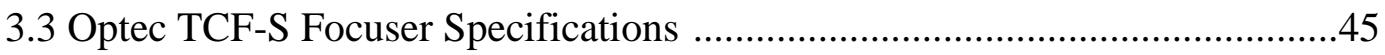

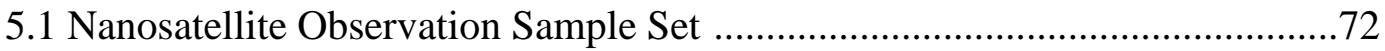

5.2 Comparison of COEs through angles-only IOD to reference TLE ................74

5.3 Comparison of Propagated State Vectors..................................................76

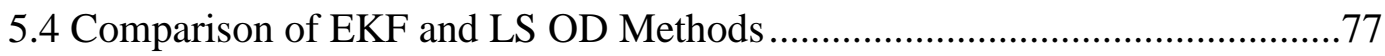

5.5 Comparison of Mean Percent Error ….....................................................8

5.6 Fmincon Optimization of Globalstar M026 Transit ...................................81

5.7 Cross-Track and Along-Track Positional Error of Satellite Trajectories ........83

A.1 All observations positional data in both equatorial and horizontal coordinate systems with time stamps in UTC

A.2 Comparison star image pixel coordinates, right ascension, and declination for each usable satellite image

A.3 Usable satellite image pixel coordinates, RA and DEC.

C.1 COE comparison of observed satellites between the initial TLE, the two Gauss methods, and the double-r method 108

C.2 CTE and ATE values for observed satellites along with the target and observed RA and DEC. Target RA and DEC provided by STK software 109 E.1 Uncertainties in the determined RA, DEC, CTE, and ATE values caused by error inherent to the pixel selection process 


\section{LIST OF FIGURES}

Figure Page

1.1 Raven in Antarctica Continuous Pixels .......................................................5

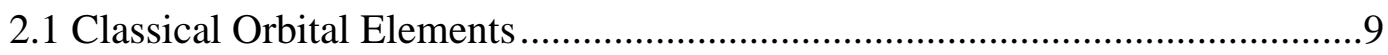

2.2 Two Line Element Set ........................................................................11

2.3 Equatorial Coordinate System .............................................................13

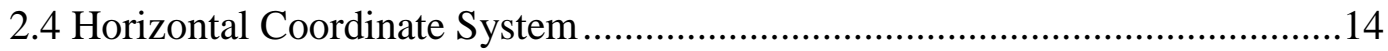

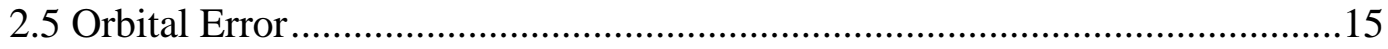

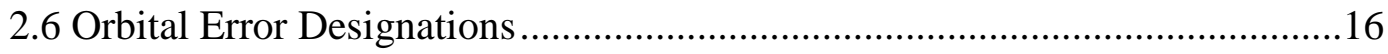

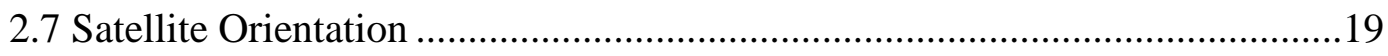

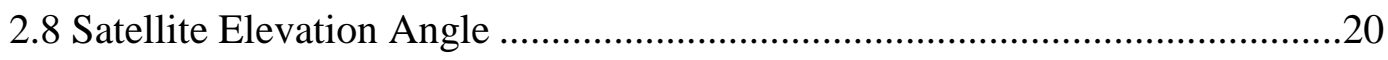

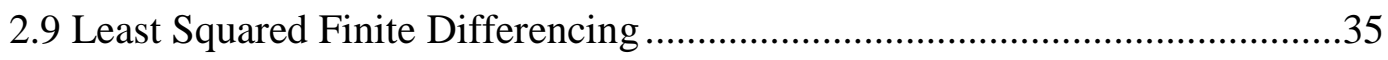

2.10 Extended Kalman Filtering Method..........................................................

3.1 Meade LX600-ACF 14" Telescope ........................................................41

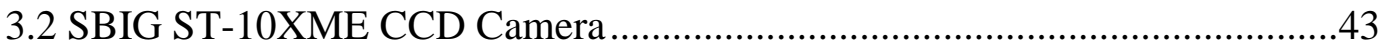

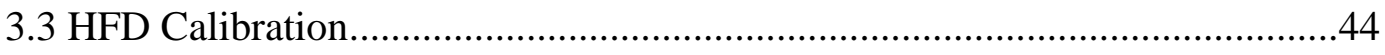

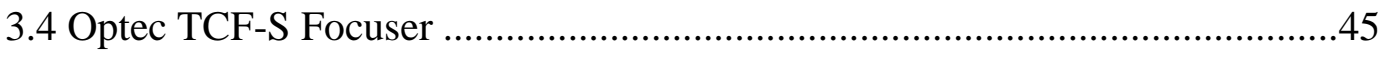

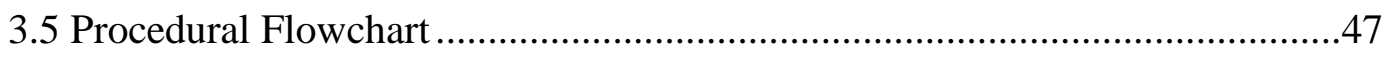

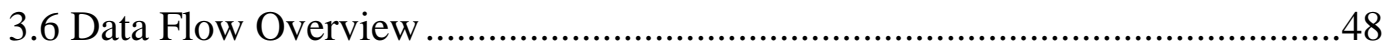

3.7 Satellite Relative Size and Dimensions .................................................50

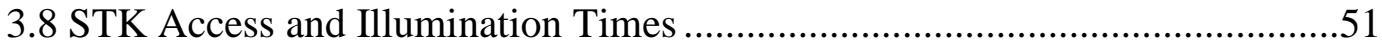

3.9 Determination of Imaging Times …..................................................52

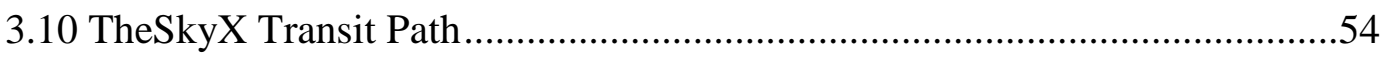

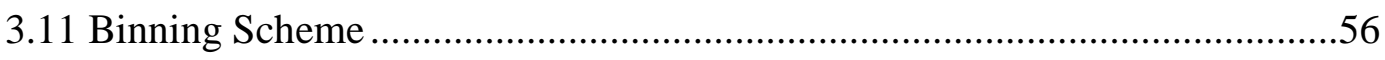

3.12 Photometric Reduction Process ...........................................................57

4.1 Star Constellation Pattern Recognition ......................................................60 
4.2 ds9 Image Viewer and Pixel Location ......................................................61

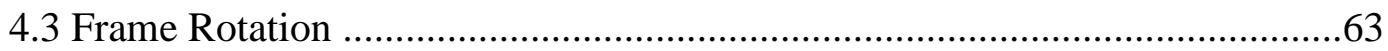

4.4 Satellite Motion and Pixel Decomposition .................................................65

4.5 Limiting Magnitudes of a Staring System ..............................................69

4.6 Different Altitude Satellite Image Photons per Pixel ...................................69

5.1 Slow Temporal Error Growth Telescope Optimization.................................79

5.2 Along-Track Error and Cross-Track Error Boxplots ...................................83

5.3 Series of Seven Images of Globalstar M053 in Single Pass ...........................86

A.1 Image taken of Globalstar M037, on April 5th, 2016, at 9:19 PM PST.

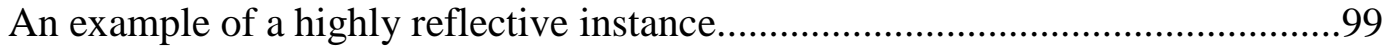

A.2 Image taken of Globalstar M030, on March 15th, 2016, at 8:27 PM PST.

This image represents the potentially dim signature of a passing satellite and can be caused by any number of reasons including solar phase angle and satellite orientation. 


\section{LIST OF ACRONYMS}

$\mathrm{ACF}$ Advanced Coma Free

AER Azimuth-Elevation-Range

ATE Along-Track Error

CCD Charge-Coupled Device COE Common Orbital Elements

CTE Cross-Track Error

DEC Declination ECEF Earth-Centered Earth-Fixed

ECI .Earth-Centered Inertial

ECS Equatorial Coordinate System

EKF Extended Kalman Filter FOV Field of View

GUI Graphic User Interface

HCS Horizontal Coordinate System

HFD Half Flux Diameter

ICRS International Celestial Reference Sysem

IOD Initial Orbit Determination IQR Interquartile Range LEO Low Earth Orbit 


MATLAB




\section{LIST OF SYMBOLS}

D

F

$J$

K

$\hat{L}_{i}$

$M_{o}$

$P$

$Q$

W

$a$

$a z$

$\tilde{b}$

$c_{i}$

$e$

el

$f, g$

$h$

$i$

$m$

$m_{A}$

$m_{P}$

$n$

pix $_{1}$

pix $_{2}$

$\vec{r}$

$\vec{r}_{\text {site }}$

$t_{E}$

$t_{i}$

$\vec{v}$

$\Delta$

$\theta$

$\Phi$ cross-track error distance

flux

cost function

Kalman gain

line-of-site unit vector

mean anomaly

error covariance matrix

second moment of the process-noise

weighting matrix

semi-major axis

azimuth

residual matrix

coefficient function

eccentricity

elevation

Lagrange coefficients

satellite altitude

inclination

apparent magnitude

slope in direction of motion od observed satellite path

slope in direction of motion of predicted satellite path

mean motion

center pixel location of satellite position 1

center pixel location of satellite position 2

position vector

position vector to point of observation

time error between observation and prediction

time at $i^{\text {th }}$ position

velocity vector

separation between star and satellite positions

angular displacement

state transition matrix 
$\Omega$

$\alpha$

$\delta$

$\mu$

$v$

$\rho$

$\tau$

$\omega$

$\S$

right ascension of ascending node

right ascension

declination

gravitational parameter

true anomaly

slant range

time relative to central position

argument of perigee

Section 


\section{INTRODUCTION}

\subsection{Purpose of Study}

The increasing pace in advancement of technology allows for modern electronics and scientific instrumentation to decrease in size and mass at an accelerating rate. Minimization of these two physical quantities within the aerospace community ultimately allow for less propulsive mass, the reduction of launch vehicle costs and the ability to transport more compact satellite bodies. Due to the driving forces of economic gain and efficient design, satellites continue to shrink in size and therefore become increasingly difficult to track from Earth's surface. This thesis is designed to determine the accuracy in which the 14" aperture Meade LX 600-ACF optical telescope located at the California Polytechnic State University (Cal Poly) can find and track satellites on the nano scale in order to determine the orbital positions of subsequent revolutions. Since 2006, Cal Poly has been launching student made CubeSats and have only been observed through acquisition tracking using a directional antenna located on site or by an off-site large optical telescope database. 
Each of these two methods have their own specific inherent drawbacks. Acquisition tracking is considered a passive tracking system using a directional antenna pointed in the general area of the satellite's pass and must rely on the CubeSat to notify the receiver station with a beacon signal. This is less than desirable due to a relatively large area that the directional antenna covers. Since this tracking system's main purpose is to communicate with the CubeSats and not determine their orbit, it is not necessary for the antenna's pointing accuracy to be as precise as an optical telescope. The optical observation database is considered an active tracking system that allows the observer to dictate the point of observation based off of both previous observations and orbital determination algorithms. The issue with this method, in the case of tracking university launched nanosatellites, is that the larger optical telescopes in which these measurements are taken usually consider smaller satellites as a lower priority. This in turn implies that the Cal Poly CubeSats do not get observed as regularly or accurately enough to retain a high fidelity propagation of its orbit. The lower prioritization of these observations may also equate to an improper cataloged identification or simply tagged as a generic object with its time and state. This could create issues when trying to decipher which observation corresponds with a precise nano-satellite, especially in the case of a simultaneous multiple CubeSats ejection.

Using the Cal Poly observatory and the previously collected Two Line Element sets (TLE) the goal of this thesis is to determine the feasibility of observing the Cal Poly launched CubeSats and comparing the predicted orbits using on-site observational data to previously determined orbital propagations. 
With the ability to observe Cal Poly launched satellites using the 14" aperture Meade LX600 on campus, independent active tracking and orbital determination of specific highinterest satellites could be feasible.

\subsection{Literature Review}

One of the first observatories to track satellites was the Asiago Astrophysical Observatory in Italy, where over a dozen satellites were observed from September 1960 to August 1962 and photographed using a moving film technique with a 0.4-1.0 meter diameter aperture. With their optical instrumentation, the lower limit of a 3.5 apparent magnitude was achieved with a stationary camera, whereas a tracking method attained a lower limit of 7-8 apparent magnitude by providing a sustained exposure along a $7^{\circ}$ arc (see $\$ 2.4$ for explanation on apparent magnitude) [1]. The technological limitations at the time affected the accuracy of the observations but steadily continue to minimize the error in the orbital predictions as telescope control increases in precision, charge-coupled devices (CCD) replaced film, and the orbital determination techniques improve.

The use of optical observational telescopes for orbital determination of satellites now spans the globe and run continuously in order to track orbiting bodies with the highest precision possible. By 2010, 33 telescopes at 23 observatories in 11 countries were operating around the world with over 90 researchers [24]. The current tasks include regular GEO monitoring, new object discovery and tracking, and maintenance of a most comprehensive catalog of orbiting bodies as possible. For Additional material on the history and development of telescopes and satellite observation, Observational Astronomy 
by D. Scott Birney and Fundamentals of Astrodynamics and Applications by David Vallado provide a thorough overview of each subject respectfully [7] [27].

Since there is a continuously increasing surplus of Earth orbiting satellites, the United States Air Force is currently working in tandem with various organizations on an alternative to large observatories surveillance for all orbiting objects. The "Raven Automated Small Telescope System" is being developed and tested by the Air Force Research Laboratory in response to the influx of satellite launches. These smaller autonomous tracking telescopes are designed to be set up around the world and routinely monitor specific segments of the night sky for passing objects.

In the paper titled Sizing of a Raven-class telescope using performance sensitivities by Ryan D. Coder and Marcus J. Holzinger [3], the correlation between the aperture diameter of a telescope, the limiting magnitudes, and information content is deduced through observational testing at various locations around the world subject to different atmospheric conditions. The values seen in Fig.1.1 illustrate the findings of the paper when in optimal conditions. Subplot (a) is the correlation between all three parameters and dissected into the 2-D planes in subplots (b)-(d). The information content values depict the effect of utilizing large focal ratios and small pixel dimensions in order to minimize the aperture diameter required to achieve a higher limiting magnitude. This limiting magnitude marks the extent of the useful observable data able to be collected with a telescope of a given aperture-size. These parameters are applicable to the specific instrumentation used in this thesis to determine the feasibility of collecting useful data near the limiting magnitude outlined in the Raven-class telescope studies. 


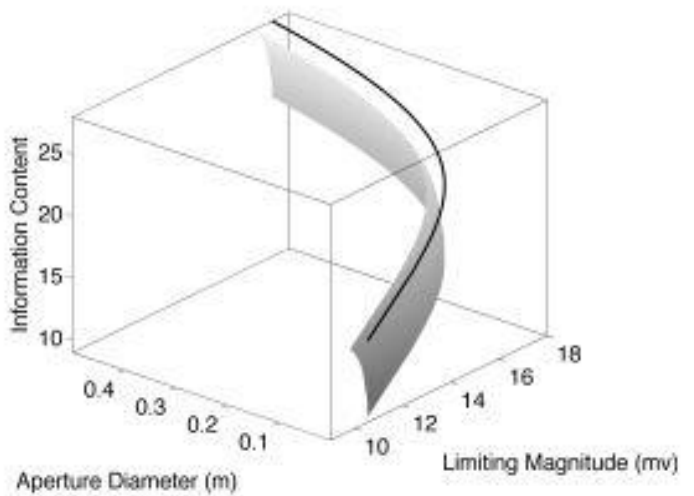

(a) Pareto Surface

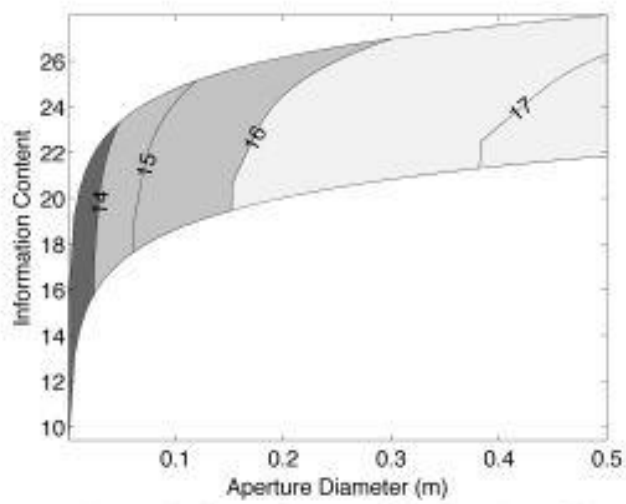

(c) Pareto Surface as Contour of Limiting Magnitude

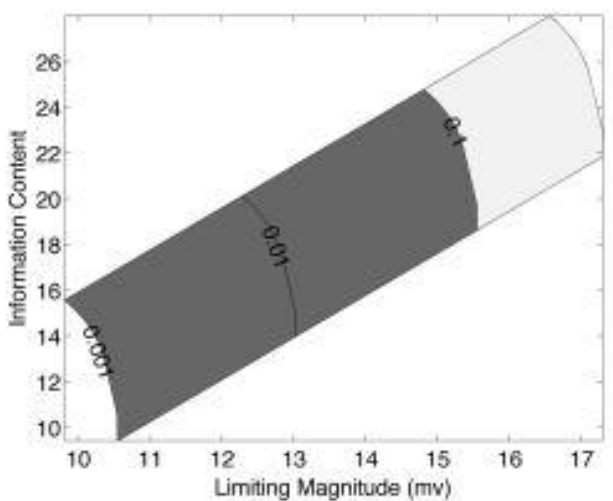

(b) Pareto Surface as Contour of Diameter

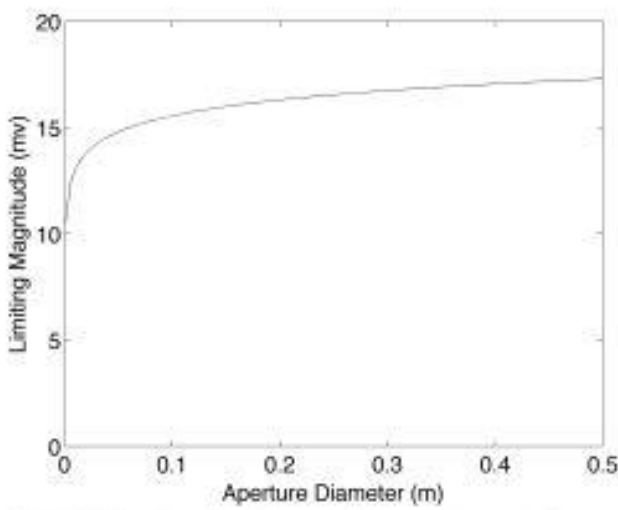

(d) Limiting Magnitude Dependence on Aperture Diameter

\section{Figure 1.1: Raven in Antarctica Continuous Pixels [3]}

In congruence with the findings presented in Fig. 1.1, Cal Poly graduate Brock Schmalzel submitted similar findings in The Feasibility and Application of Observing Small LEO Satellites and Amateur Telescopes upon which this thesis is a continuation [19]. Some of the topics researched in this thesis were preliminary angles-only orbit determination, the generation of a satellite brightness prediction model, astrometric analysis to provide a measurement of temporal error growth in TLE-based orbit propagation, and an optimization of an ideal telescope for LEO observations. The ideal telescope dimensions for LEO observations were determined through the use of the brightness prediction model and data from the TLE temporal error growth. 
The brightness prediction model presented in Schmalzel's paper is based on the apparent magnitude of an object (covered in more detail in §2.4) and photometric analysis. Differential photometry was used in order to determine an upper and lower range of magnitudes satellites in 96 observations in contrast to an individually chosen comparison star. These values were then verified with the N2YO online database and used in telescope optimization. In addition to the magnitude prediction model, the second factor in optimizing the telescope's dimensions were an analysis of the TLE temporal error growth.

The temporal error growth consisted of the observation of nine satellites over a multiple day span while being referred from a single initial TLE of a consistent epoch. The deviations in both the cross-track and the along-track error of the satellites were recorded for each observation. Although there were relatively major deviations from expected values in the cross-track error of the satellites, the along-track errors was within the expected boundaries (an explanation of cross-track and along-track errors can be found in §2.1.5). Both a slow and fast temporal growth estimation were used to determine the two extremes of the desired telescope dimensions [23].

The telescope optimization was ran for three test cases for satellites of different sizes. Although there were relatively larger differences between the lower and upper bound of desired sizing for the telescopes due to the slow and fast TLE temporal growth, there are clear trends shared with the research conducted by the Air Force Research Lab. The results of Schmalzel's findings will be discussed in congruence with my own in Chapter 5.

Although Brock Schmalzel conducted his observations at the Cal Poly observatory as well, the observing instrumentation differs. The Meade LX 200 12" telescope was replaced with the current Meade LX 600 14" telescope. With the increased aperture size 
and its correlation with limiting magnitude previously discussed, the ability to image smaller satellites is more feasible. The additional differences in the instrumentation used in Brock's observations and this thesis will be covered in $\S 3.1$.

In addition to the correlation of the data collected between both telescopes used to verify the improvement in accuracy and feasibility of determining the propagated satellite orbits, I will also be comparing the procedural differences taken in the acquisition of the satellite images to increase the amount of data points within a single pass.

\subsection{Structure of Paper}

Review of orbital mechanics, orbital determination methods, and apparent magnitudes will be in the following chapter. A general overview of these topics will assist in the understanding of the thesis goal, data analysis, and conclusion. Chapter 3 will contain an overview and specification lists on the instrumentation used in observation as well as explain the procedures on the preparation of observation, the observation process itself, the

photometric reduction, and the orbital determination processes. Chapter 4 will go over the analysis underwent in the astrometry aspects of the images taken. Chapter 5 will contain the results and discussion and Chapter 6 will be the conclusion of the thesis with recommendations for future work on this subject. 


\section{ASTRODYNAMICS BACKGROUND}

\subsection{Orbital Mechanics Review}

If you are not fully familiar with orbital dynamics, celestial coordinate systems, and two line element sets, it is suggested to review $\$ 2.1$ otherwise continue on to $\S 2.2$.

\subsubsection{Classical Orbital Elements}

Classical Orbital Elements (COEs) are the parameters in which the positioning of all objects in space are defined when portrayed in their "two line element set" (TLE). The COEs of an object are a series of six Keplerian elements which accurately mark an object at a specific point in space as well as define the path in which it is travelling. These six elements are the eccentricity, semi-major axis, inclination, right ascension of ascending node, argument of perigee, and the mean anomaly. Each one of these parameters defines a specific attribute of an object's orbit and/or position within their orbit. All of these parameters can be seen in Figure 2.1. 


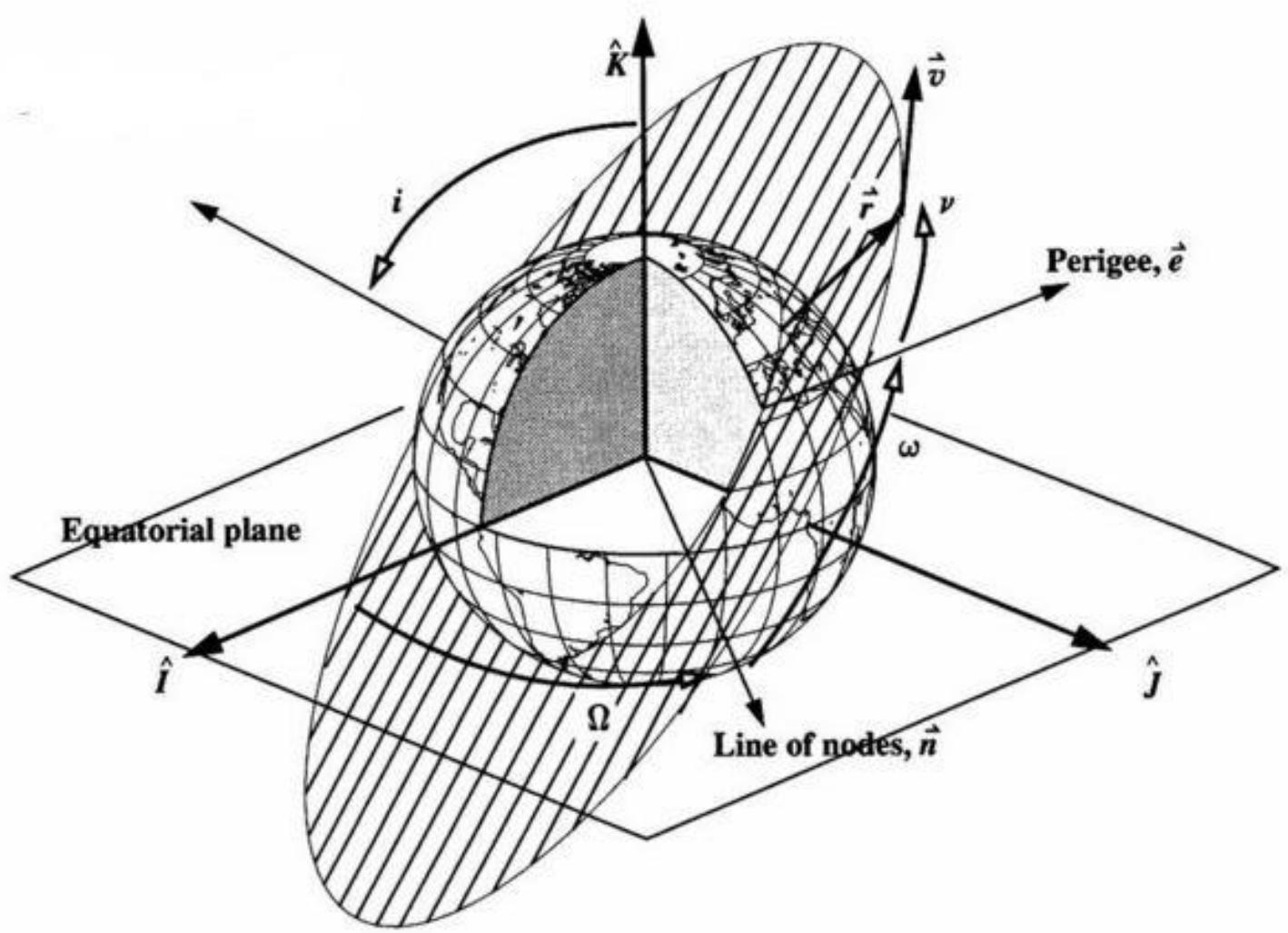

Figure 2.1: Classical Orbital Elements [2]

An orbit's eccentricity $(e)$ is the value defining circularization around its host body and depicted as:

$$
e=\frac{r_{a}-r_{p}}{r_{a}+r_{p}}
$$

Where $r_{a}$ is the apogee radius of the orbit and $r_{p}$ is the perigee radius. A perfectly circular orbit is defined as 0 eccentricity, elliptical orbits have an eccentricity between 0 and 1 , and any eccentricity value over 1 is considered unbounded and in an escape trajectory. Since all observed objects in this thesis are in nearly circular LEO orbits, the eccentricity will effectively be 0 . 
The semi-major axis $(a)$ is the sum of the periapsis and apoapsis distance divided in half. It is an indication of an orbits size and shape in accordance with the orbits eccentricity. In the case of this thesis, the semi-major axis of all observations will be located in the LEO classification which lies between $6,530 \mathrm{~km}$ and 8,370 $\mathrm{km}$ from Earth's center.

The vast majority of the semi-major axis associated with the observations will lie near the lower boundary of this region due to the average deployment altitude of most LEO satellites.

The inclination $(i)$ depicts the vertical tilt of the ellipse in relation to the reference frame along the equatorial plane as depicted in Fig. 2.1. The inclination of an orbit is measured along the ascending node, the location in which the object passes upward through the reference plane.

The Right Ascension of the Ascending Node or RAAN $(\Omega)$ orients the location of the previously mentioned ascending node horizontally along the equatorial plane from the vector directed toward the First Point of Aries. In Fig. 2.1, this can be seen as the vector denoted by $\hat{\mathrm{I}}$.

The argument of perigee $(\omega)$ defines the orientation of the ellipse within the orbital plane. This angle is referenced from the ascending node to the perigee of an orbit. In tandem with this angle, the true anomaly $(v)$ of a body in space is the angle defined from $\omega$ to the object at a specific epoch. 


\subsubsection{Two Line Element Sets}

A TLE is the standard in defining the position of an object in space at a specific epoch. Every observation taken of an object can be reduced to its COEs. These elements are depicted in a set of two lines which also includes the object ID, epoch, and revolution number. An example of a TLE and defined values can be seen below in Figure 2.2.

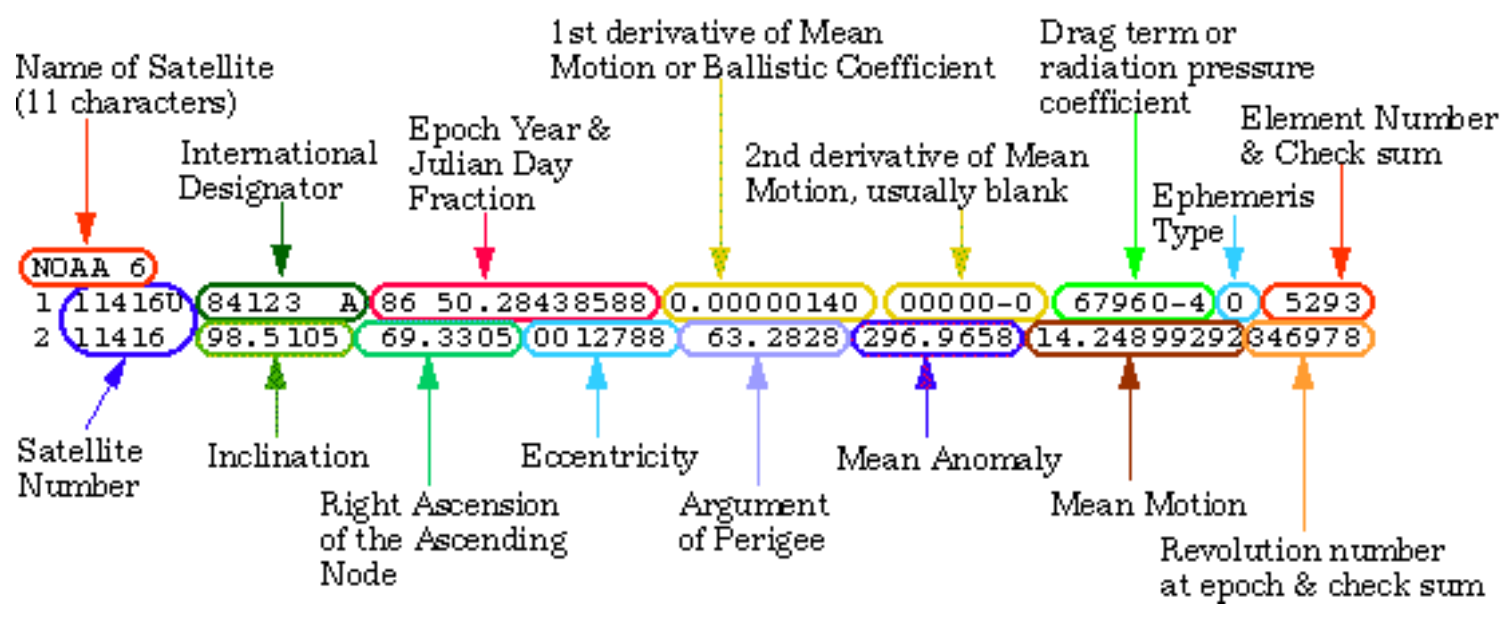

Figure 2.2: Two Line Element Set [5]

Each of these values indicated in the TLE in Fig. 2.2 serve a purpose in depicting the qualities of the observed object. TLEs are produced for all observed objects in space and are constantly updated to ensure the most accurate position and velocity vectors. As more time passes from when a TLE of an object was cataloged, it begins to degrade in fidelity due to applied perturbations ad external forces. These forces can create an altered orbital path from the previously predicted trajectory. The only reliable way to negate the increasing divergence of the predicted orbit from the true orbit is to minimize the time that has passed from the last observation point. With a shorter time frame between the data points, the compounding effects of the external forces acting upon the object are minimized. 


\subsubsection{Alternative Position Systems}

In addition to COEs, another commonly used form of defining the location of an objects motion is through the position and velocity vectors. As seen in Fig. 2.1, the two vectors denoted by $\vec{r}$ and $\vec{v}$ indicate the position and velocity of an object at a specific moment in time. These vectors comprise of three values, each defined in the cardinal

coordinate directions $\hat{I}, \hat{J}$, and $\widehat{K}$. The $\vec{r}$ and $\vec{v}$ vectors and/or COEs can be determined through the manipulation of the other by means of coordinate transformation or vector algebra. The position and velocity vectors are a useful and intuitive means of describing the motion of an object. Satellite locations are determined relative to the position of the observer and Earth's center through vector quantities. An understanding of both the previously defined positioning systems as well as conversion between the two is necessary for the observations to be captured, analyzed, and cataloged.

\subsubsection{Celestial Coordinate Systems}

When taking observations in a perceived 2-D spherical plane, the coordinate systems most commonly used are the Celestial Equatorial Coordinate System and the Horizontal Coordinate System. Each of these systems utilize two directional values while the third coordinate value needed is "range", which is determined through other means covered in $\$ 2.2$.

The Equatorial Coordinate System (ECS) defines position through right ascension (RA) and declination (DEC) on the celestial sphere while the Horizontal Coordinate System (HCS) defines position using azimuth $(A z)$ and elevation $(E l)$. Both coordinate 
systems can be used to define the same position with the key difference in the datum point of which the frame is based. The ECS is based off of the Earth's equator with the vernal equinox defining $0^{\circ}$ DEC and Ohrs RA respectively. The DEC utilizes the degrees/minutes/seconds spherical units while the RA usually utilizes units of hours/minutes/seconds spherical units. DEC ranges from $90^{\circ}$ above the equator to $-90^{\circ}$ below while the RA of an object increases eastwardly from the vernal equinox starting at Ohrs to $24 \mathrm{hrs}$ as seen in Fig. 2.3. For the purposes of this thesis, the ECS coordinates are constant and do not change over time.

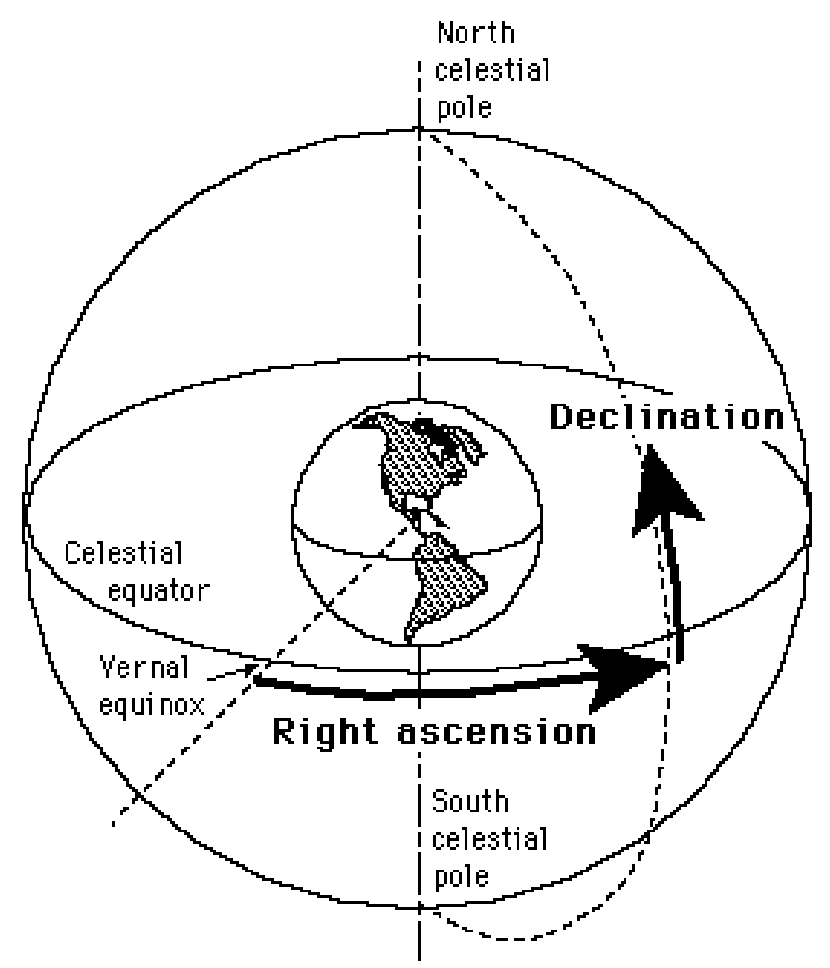

Figure 2.3: Equatorial Coordinate System [12]

In Fig. 2.4, the HCS alternatively uses the observer's specific horizon line on Earth's surface as the reference point which revolves around its own axis once per sidereal day. Since the Azimuth of an object is based off of the north facing direction (increasing from $0^{\circ}$ to $360^{\circ}$ westward), all celestial objects have varying positional coordinates since it 
is based off of the Earth's rotational reference frame. Due to the fact that this coordinate system is based off of the observer's horizon line, the elevation of an object varies from $0^{\circ}$ to $90^{\circ}$ from horizon to zenith. This coordinate system is useful for observers due to the quantifiable orbit locations specific to the observer's immediate location unlike the more general time invariant Coordinate System.

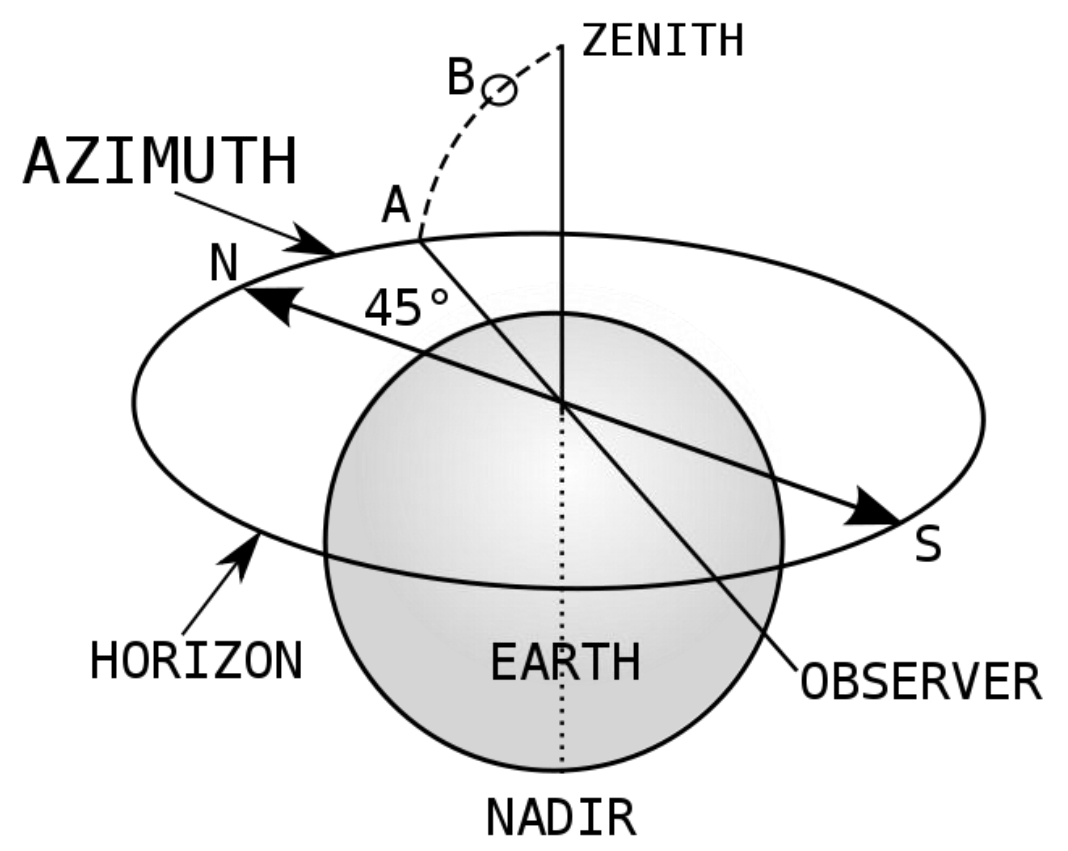

Figure 2.4: Horizontal Coordinate System [4]

Both coordinate systems utilize the spherical units of degrees, minutes, and seconds, but have their own unique benefits. The ECS is commonly used in celestial body databases such as the VizieR Astronomical Library and the SIMBAD Astronomical Database due to the convenience of cataloging the permanent location of astronomical bodies such as stars and galaxies referenced to our equator. The HCS is used in satellite databases such as CelesTrak.com and n2yo.com due to the constantly changing position of satellites relative to any specified observation location on Earth's surface. Both coordinate 
systems must be utilized when taking observations of satellites due to the required satellite positions relative to the Cal Poly observatory as well as to the celestial bodies in the background of the images taken.

\subsubsection{Orbital Error}

One of the main reasons in attempting to observe Cal Poly CubeSats is to increase the accuracy of the orbital propagation predictions of the satellite's locations. In Fig. 2.5, you can see the representation of a notable difference in an estimated trajectory versus the true trajectory of the satellite.

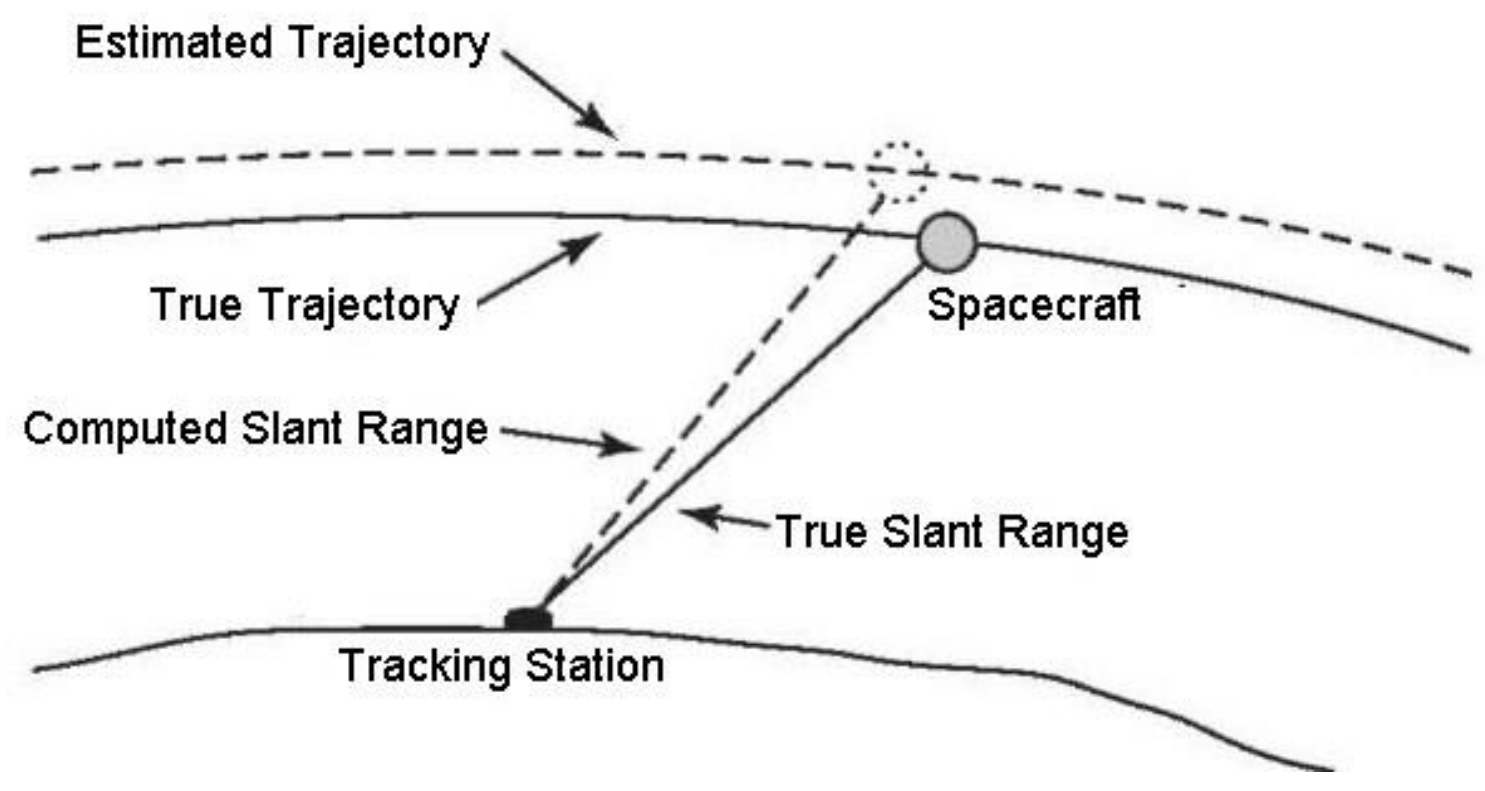

Figure 2.5: Orbital Error [32]

There are many reasons that can cause the variance of an orbiting body from the true trajectory. Atmospheric drag, solar radiation pressure, and oblateness are a few of the most influential external forces. These forces compound over time and push the satellite further off of the predetermined trajectory based on previous observations. It is the goal of satellite observation systems to continuously monitor satellites in order to mitigate these 
altering effects and accurately predict where satellites are located at all times to within a suitable tolerance of error.

Although there are multiple ways in which an orbital path can be affected by outside sources, all of these influences can be culminated into two quantifiable errors. These two errors are cross-track error (CTE) and along-track error (ATE), as seen below in Fig.2.6.
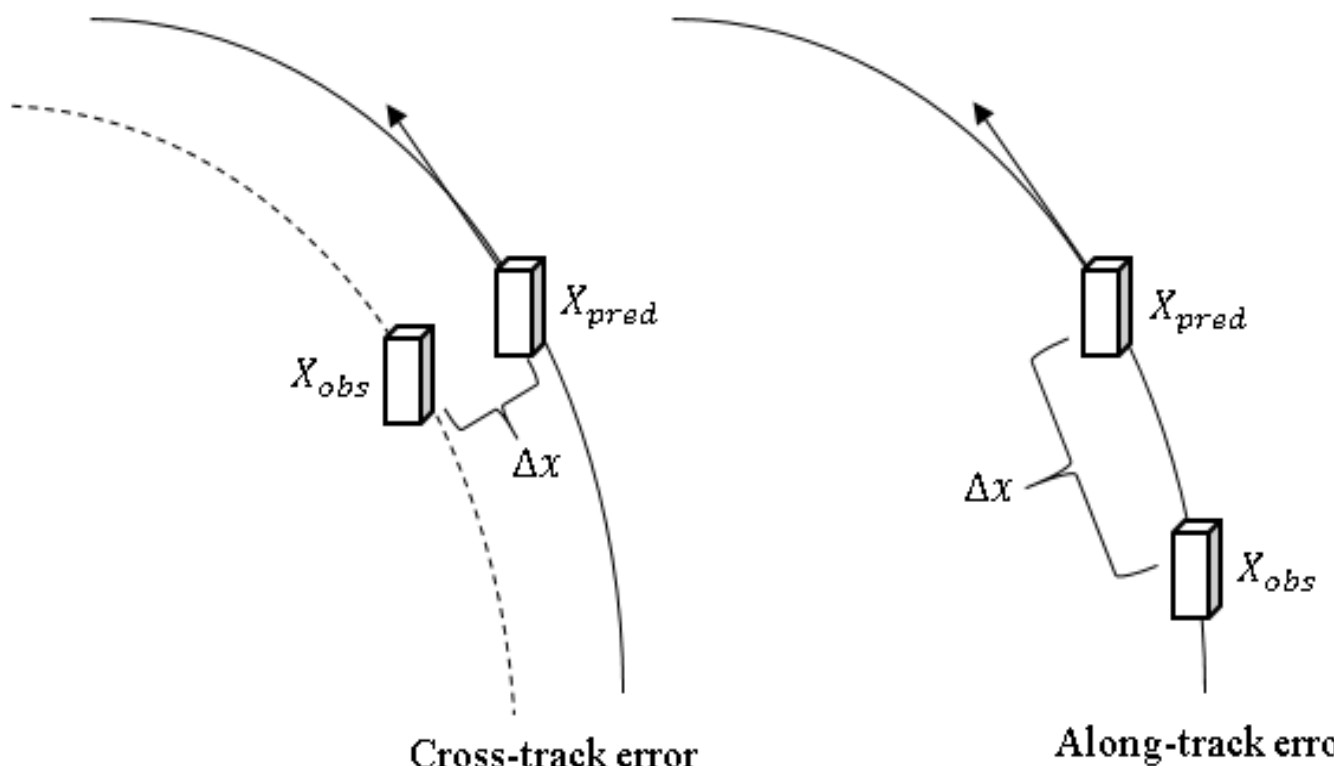

Cross-track error

\section{Along-track error}

\section{Figure 2.6: Orbital Error Designations}

Cross-track error is an indication of the variance from the predicted path of the orbiting object in the plane perpendicular to the direction of motion, while along-track error adversely is the variation within the direction of motion. External perturbations have an effect on both orbital error distinctions, but the along-track error is predominately affected by forces in low Earth orbits. Since LEO objects are relatively close to the upper atmosphere, the associated drag forces overwhelm the other perturbation forces slowing the satellite's orbital velocity and affecting the predicted along-track location. A quantity 
in either of these errors larger than the Cal Poly telescope field of view (FOV) of 22'x15' arcminutes result in an unsuccessful observation attempt. In order to mitigate the chance for these missed observations, it is imperative to use the most recent TLE data available.

\subsection{Satellite Magnitudes}

When attempting to observe objects at a distance, there are many effects that can hinder the perceived brightness. Two values are used to quantify an objects brightness are the absolute magnitude and apparent magnitude. In addition to these two values, there is also an instrumental magnitude associated with the observing hardware. The instrumental magnitude is the uncalibrated apparent magnitude registered by the CCD camera based on the photon count and number of pixels affected, depicted in Eq.2.0a below.

$$
m_{\text {inst }}=A-2.5 \log _{10}\left[\frac{\left(\sum_{i=1}^{n} C_{i}\right)-n C_{s k y}}{t}\right]
$$

The variables $C_{i}$ and $C_{s k y}$ represent the photon count captured by the object and the ambient photon count of the annulus surrounding the object respectively. The variables $n$ and $t$ are the pixel count and exposure time while $A$ represents an added arbitrary constant. The telescope, CCD limitations, atmospheric scintillation and seeing effects are cause for the differences between the instrumental magnitude and apparent magnitudes [34].

The absolute magnitude of an object is the determined brightness at a common distance of ten parsecs from Earth. When theoretically forcing all objects to be on the same plane, it is effectively removing the influence of distance on an object's brightness and putting all astronomical bodies on an even scale depicting their intrinsic luminosity. 
Apparent magnitude is the perceived brightness of an object when taking their distance from the observer into account (all references to magnitude in this paper pertain to apparent magnitude). The apparent magnitude is based off of the flux output of an object. An objects flux decreases at a proportion inversely squared to its distance from the observer.

The satellite's apparent magnitudes will be used to determine the feasibility of observing nanosatellites from the observatory here at Cal Poly since the LEO slant-range distance is a major factor in observation feasibility. The simplest way to determine an object's magnitude is known as differential photometry. This method is based off of the comparison of all observations to a specific reference star defined by Eq.2.0b [17].

$$
m-m_{0}=-2.5 \log _{10}\left(\frac{F}{F_{0}}\right)
$$

The apparent magnitude of an orbiting object must be referenced with respect to the magnitude and flux value of the previously known object, depicted by $m_{0}$ and $F_{0}$ respectively.

Some of the main factors contributing to the apparent magnitude of a satellite's perceived brightness are the solar phase angle (SPA), atmospheric seeing conditions, satellite albedo, satellite orientation, satellite relative velocity, and the size of the satellite being observed. The solar phase angle of the satellite can severely diminish the flux of the satellite if misaligned with the Sun and Earth and/or the geometry of the object is not conducive to reflection. If the satellite is at an angle to the Sun and the observer's position that does not effectively reflect the sun's light, it will usually render the satellite unobservable as seen in Fig.2.9. 


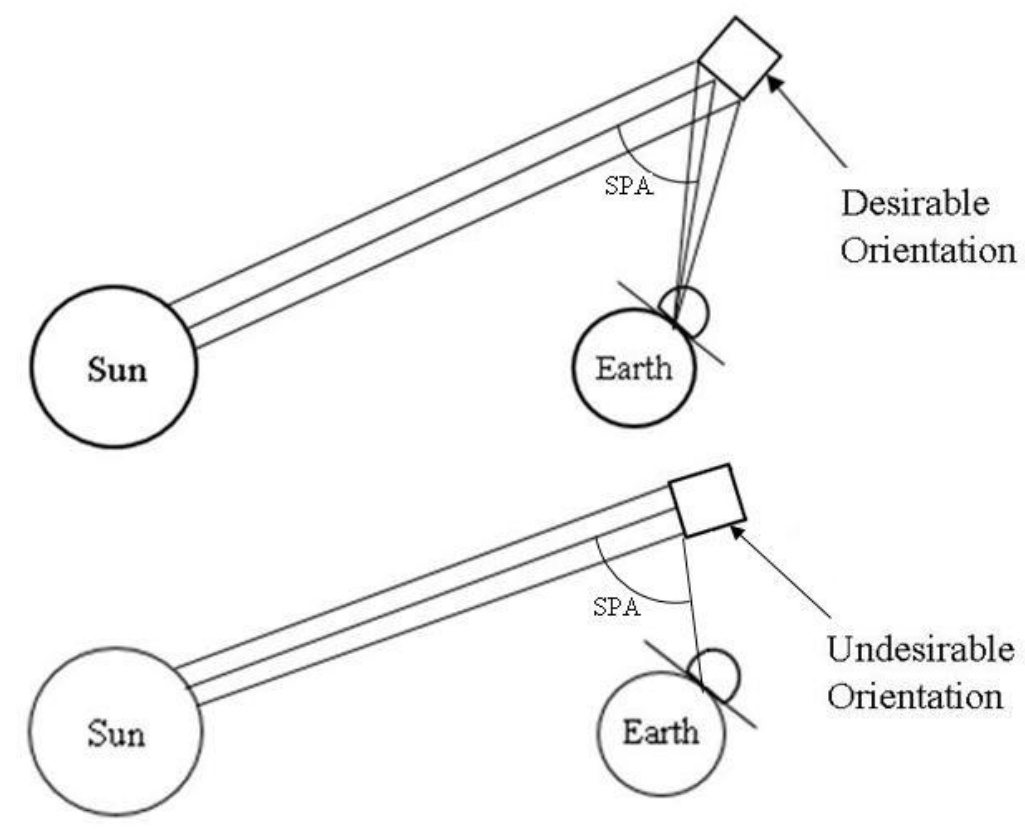

Figure 2.7: Satellite Orientation

The SPA dictates the required elevation of the satellite above the horizon and time of the observation needed for a successful observation. The solar phase angle can be calculated though vector algebra seen below, where the variables $\vec{r}_{S A T}, \vec{r}_{\text {SITE }}$, and $\vec{r}_{\text {SUN }}$ are the position vectors of the satellite, observatory, and Sun.

$$
\begin{gathered}
\vec{r}_{A}=\vec{r}_{S A T}-\vec{r}_{S I T E} \\
\vec{r}_{B}=\vec{r}_{S A T}-\vec{r}_{S U N} \\
S P A=\cos ^{-1}\left(\frac{\vec{r}_{A} \cdot \vec{r}_{B}}{\left\|\vec{r}_{A}\right\|\left\|\vec{r}_{B}\right\|}\right)
\end{gathered}
$$

Since the satellite cannot be seen if eclipsed by the Earth's umbra, the highest yield of successful observation occurs from to roughly 11:00 pm PST. Once past 11:00 pm, the majority of the satellite's observable path is shadowed and inhibits the satellite in reflecting enough of the Sun's light toward the telescope, depicted in Fig. 2.10. 


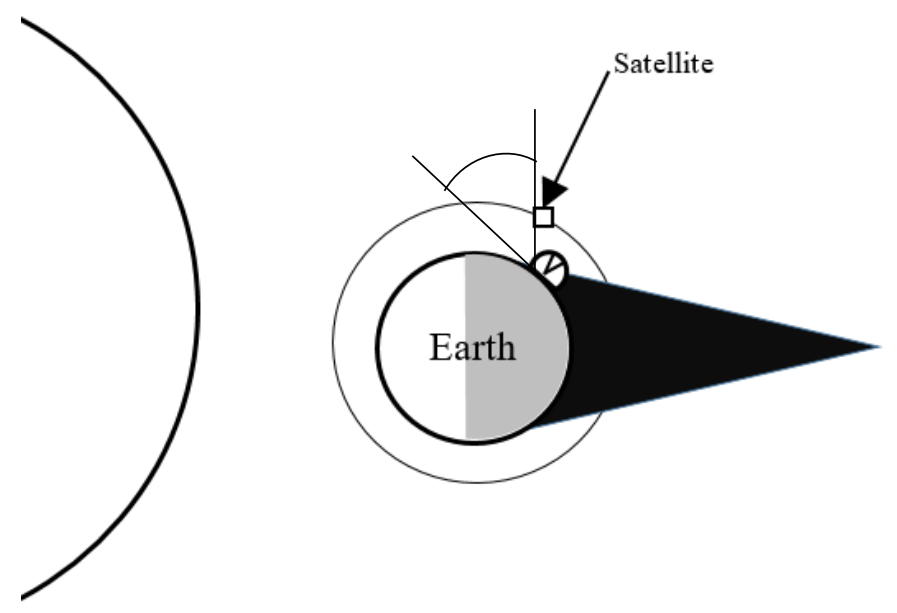

Figure 2.8: Satellite Elevation Angle

The flux of a passing satellite is maximized when in the correct phase angle with the Sun and observer. The satellite's albedo is dictated by the outer material's reflectance. Although most satellites are usually coated with high-albedo insulating thermal blankets, CubeSats are not due to the irrelevance for such a relatively short lifespans. However, an advantage of observing nanosatellite is the fact that most are injected into a LEO orbit; this minimizes the distance to the object and increases the apparent magnitude. Even with an increase in albedo and a relatively low orbit, nanosatellites are orders of magnitude smaller in cross-sectional area than the average satellite, making observation significantly more difficult.

With these restrictions and constraints, the areas in which observations can occur are limited and extremely dim with respect to larger satellites. As the perceived brightness of the observed object increases, the apparent magnitude value becomes more negative. Table 2.1 lists a few apparent magnitudes of known objects as well as calculated apparent magnitudes of observed satellites. 
Table 2.1: Apparent Magnitude Values

\begin{tabular}{|c|c|}
\hline Apparent Mag. & Observed Object \\
\hline-26.74 & Sun \\
\hline-12.90 & Full Moon \\
\hline-2.70 & Jupiter \\
\hline 9.4 & Globalstar Satellite \\
\hline 12.6 & 3U CubeSat \\
\hline$\sim 14.0$ & Maximum Observable Apparent Mag. Of Telescope \\
\hline
\end{tabular}

Looking at Eq.2.0, you can see that the magnitude of an object increases logarithmically. The increase in apparent magnitude of 1.0 correlates to the object being roughly 2.5 times dimmer. Using this equation, the theoretical apparent magnitudes of a nanosatellite as well as a Globalstar satellite under ideal conditions can be calculated. Although the ideal apparent magnitude of a nanosatellite is close to the maximum observable apparent magnitude of the Meade LX600-ACF 14" telescope, it is still an order of magnitude under the limit. This strengthens the possibility of successfully being able to observe and track CubeSats in LEO orbits.

\subsection{Initial Orbit Determination}

Since Karl Gauss first derived the formulas in which angles-only initial orbit determination (IOD) is based, many different solutions have been formed to solve the same problem. Some examples include the Double-r method, Gooding's method, and the Herrick-Gibbs method. Each of these solutions have different advantages and disadvantages to be taken into consideration based on the characteristics of the set of data 
points collected which will be discussed later in Chapter 2. Both the mathematical reasoning and implementation of these methods were thoroughly explained in Fundamentals of Astrodynamics and Applications by Vallado [26]. IOD methods discussed in this text are analytical approaches in determining position and velocity vectors through the processing of raw data before determining an orbit. Although differential correction is used to determine the orbit of an object, the data could not be processed without the individual vectors determined through these techniques.

There are many IOD models that differ drastically in functionality. The angles-only method for initial orbital determination will be used throughout this thesis due to the sole use of a single Meade LX 600-ACF optical telescope. Angles-only observation requires the use of only the azimuth and elevation of an orbiting body. Other IOD models include range, range-rate, and mixed observational methods. These methods require multiple sensor outputs and simultaneous observations. The two methods that will be used are the Gauss Method and the Double-r Method. These angles-only methods work for angular data but are fundamentally limited due to the lack of knowing the range of the orbiting body. As you can see in Fig. 2.5, the slant range $(\rho)$ of an object is the positional vector quantity of the satellite with respect to the observation site [27]. The slant range distances of the objects being observed in this thesis range from 1500 to $3000 \mathrm{~km}$ for Globalstar satellites. The CubeSat slant ranges are significantly lower at roughly 800-1000 km distances which consequently require these satellites to travel much faster.

Before each method is implemented, there is underlying data that must first be collected. Since the observations are taken in the horizontal coordinate system, the location of the observer is both an important position and can be defined to a high degree of 
precision. The position vector from the center of mass of the Earth to the designated position of the observer is denoted as $\vec{r}_{\text {site. }}$ This position vector in addition to the slantrange vector seen in Fig. 2.5 creates the $\vec{r}$ vector spanning from the center of the Earth to the position of the observed satellite. These $\vec{r}$ values act as the base data values of the angles-only IOD methods. The IOD methods discussed include three ordered pairs of observations in RA and DEC as well as the times of the observations. The RA $(\alpha)$ and DEC $(\delta)$ of each observation can then be used to form a line-of-site unit vector $\left(\widehat{L}_{\mathrm{i}}\right)$ using Eq.2.1 below.

$$
\hat{L}_{i}=\left[\begin{array}{c}
\cos \left(\delta_{t i}\right) \cos \left(\alpha_{t i}\right) \\
\cos \left(\delta_{t i}\right) \sin \left(\alpha_{t i}\right) \\
\sin \left(\delta_{t i}\right)
\end{array}\right] \quad i=1,2,3
$$

If the slant range ( $\rho$ ) were known, the $\vec{r}$ values of each observation can be determined through Eq.2.2.

$$
\vec{r}=\rho \hat{L}+\vec{r}_{\text {site }}
$$

Implementing the dot product on Eq.2.2, the magnitude of $\vec{r}$ seen in Eq.2.3 is acquired.

$$
r=\sqrt{\rho^{2}+2 \rho \hat{L} \cdot \vec{r}_{\text {site }}+r_{\text {site }}{ }^{2}}
$$

Since the slant-range values of the observations are unknown at this point, the values must be iterated upon using the positional data collected [27]. 


\subsubsection{Gauss Method}

The Gauss Method is the first IOD that will be used in predicting the motion of the orbiting bodies. In relation to other IOD methods, it is relatively simple and straightforward to implement. This method works best when the angular separation between observations is less than $\sim 60^{\circ}$ and performs remarkably well when the data is separated by $10^{\circ}$ or less. This angular separation translates to observations that are about two to five minutes apart for LEO satellites. When properly formulated, Gauss's routine is a modestly robust way to determine a satellites position with angles-only data. IOD methods require a minimum of three observation points so that the slant-range of the central observation can be determined through the leading and trailing observed positional unit vectors. Once the slant-range is determined, all three positional dimensions are defined and the initial orbit can be calculated [27].

This technique relies on a safe assumption that all three required observations lie on a single plane, which can be written as Eq.2.4, where $\vec{r}_{i}$ is the position vector and $c_{i}$ is the respective coefficient function.

$$
c_{1} \vec{r}_{1}+c_{2} \vec{r}_{2}+c_{3} \vec{r}_{3}=\overrightarrow{0}
$$

Since the current interest is in the relation between these vectors and not the quantity, rescaling the coefficients for convenience is desirable. By setting $\mathrm{c}_{2}=-1$, the derivation of the coefficient functions is simplified. Through the use of the series form of the Lagrange coefficients and taking the cross product of the first and third positions, the coefficient function forms are determined to be Eq.2.5 and Eq.2.6.

$$
c_{1}=a_{1}+a_{1 u} u
$$




$$
c_{3}=a_{3}+a_{3 u} u
$$

These functions consist of the variables $a_{i}$ and $a_{i u}$ denoting the ratio of time variance of the $\mathrm{i}^{\text {th }}$ position relative to the central $\vec{r}_{2}$ time and similarly the differential in time of $\vec{r}_{1}$ and $\vec{r}_{3}$ relative to the central position $(\tau)$ is defined as:

$$
\tau_{i}=t_{i}-t_{2}
$$

Since the velocity of the object is still unknown, the series form of Lagrange coefficients need to be implemented [27]. Through mathematical manipulation, the final form of the equations for $a_{i}, a_{i u}$, and $u$ can be written as Eq.2.7-2.11, where $u$ is a newly introduced variable of the series form and $\mu$ being the gravitational parameter.

$$
\begin{gathered}
a_{1}=\frac{\tau_{3}}{\tau_{3}-\tau_{1}} \\
a_{3}=\frac{\tau_{1}}{\tau_{3}-\tau_{1}} \\
a_{1 u}=\frac{\tau_{3}\left(\left(\tau_{3}+\tau_{1}\right)^{2}-\tau_{3}{ }^{2}\right)}{6\left(\tau_{3}-\tau_{1}\right)} \\
a_{3 u}=\frac{\tau_{1}\left(\left(\tau_{3}+\tau_{1}\right)^{2}-\tau_{3}{ }^{2}\right)}{6\left(\tau_{3}-\tau_{1}\right)} \\
u=\frac{\mu}{r_{2}^{3}}
\end{gathered}
$$

Now that expressions to approximate the coefficient functions have been defined, the relation of these values to the observation slant-ranges must be determined. Using Eq.2.2, the $\vec{r}$ values in Eq. 2.4 can be substituted for that of the sum of the slant-ranges and

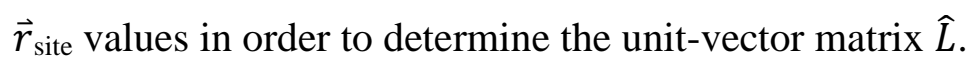


When separating the $\rho$ terms with the $\vec{r}_{\text {site }}$ terms, the form becomes:

$$
\left\langle\hat{L}_{1}\left|\hat{L}_{2}\right| \hat{L}_{3}\right\rangle\left[\begin{array}{l}
c_{1} \rho_{1} \\
c_{2} \rho_{2} \\
c_{3} \rho_{3}
\end{array}\right]=\left\langle\vec{r}_{\text {site } 1}\left|\vec{r}_{\text {site } 2}\right| \vec{r}_{\text {site } 3}\right\rangle\left[\begin{array}{l}
-c_{1} \\
-c_{2} \\
-c_{3}
\end{array}\right]
$$

In order to determine the slant-ranges of the observations, the $\widehat{L}$ matrix must be inverted through the use of Cramer's Rule to single out the $\rho$ values on one side, creating:

$$
L^{-1}=\frac{\left[\begin{array}{ccc}
L_{y 2} L_{z 3}-L_{y 3} L_{z 2} & -L_{y 1} L_{z 3}+L_{y 3} L_{z 1} & L_{y 1} L_{z 2}-L_{y 2} L_{z 1} \\
-L_{x 2} L_{z 3}+L_{x 3} L_{z 2} & L_{x 1} L_{z 3}-L_{x 3} L_{z 1} & -L_{x 1} L_{z 2}+L_{x 2} L_{z 1} \\
L_{x 2} L_{y 3}-L_{x 3} L_{y 2} & -L_{x 1} L_{y 3}+L_{x 3} L_{y 1} & L_{x 1} L_{y 2}-L_{x 2} L_{y 1}
\end{array}\right]}{|L|}
$$

Using Eq.2.5 and Eq.2.6 to substitute the coefficient functions to the $\left(a_{i}+a_{i u} u\right)$ form and redefine $M=L^{-1}\left\langle\vec{r}\right.$ site $\left._{1}\right| \vec{r}$ site $_{2} \mid \vec{r}$ site $\left._{3}\right\rangle$ the equation can be simplified and can equate the middle observation's slant-range to:

$$
\rho_{2}=M_{21} a_{1}-M_{22}+M_{23} a_{3}+\left(M_{21} a_{1 u}+M_{23} a_{3 u}\right) u=d_{1}+d_{2} u
$$

Substituting in $\mathrm{d}_{1}$ and $\mathrm{d}_{2}$ for simplification and defining $C=\widehat{L}_{2} \cdot \vec{r}$ site $_{2}$, the eighth-degree polynomial can be derived seen in Eq.2.12.

$$
r_{2}^{8}-\left(d_{1}^{2}+2 C d_{1}+\vec{r} \text { site }_{2}^{2}\right) r_{2}^{6}-2 \mu\left(C d_{2}+d_{1} d_{2}\right) r_{2}^{3}-\mu^{2} d_{2}^{2}=0
$$

By solving for the correct real root $r_{2}$ in Eq.2.12 and iterating upon the initial estimate of the slant ranges, convergence on the best fit solution within an acceptable variation tolerance can be determined. 


\subsubsection{Double-r Method}

The Double-r Method varies from Gauss's Method both in procedure and functionality. The Double-r Method is more desirable than Gauss's Method in the sense that it can handle observations that are very far apart in angular position. A starting guess for the position vector is required to initialize the iterative process used in this method.

With the initial $r_{i}$ magnitude guesses and defining:

$$
c_{i}=2 \hat{L}_{i} \cdot \vec{r} \text { site }_{i} \quad i=1,2
$$

the preliminary slant range value can be calculated using Eq.2.13.

$$
\rho_{i}=\frac{-c_{i}+\sqrt{c_{i}^{2}-4\left(r_{\text {site }}^{2}-r_{i}^{2}\right)}}{2} \quad i=1,2
$$

These values can then be multiplied with the unit-vector matrix, $\hat{L}$, and vector sum with the $\vec{r}_{\text {site }}$ values previously calculated to acquire the first iteration of $\vec{r}$ values shown as Eq.2.14.

$$
\vec{r}_{i}=\rho_{i} \widehat{L}_{i}+\vec{r}_{\text {site }_{i}} \quad i=1,2
$$

These $\vec{r}_{1}$ and $\vec{r}_{2}$ positional vectors of the observed object now define the orbital plane. Using the satellite-based Equinoctial Coordinate System, the orbital plane acts as the fundamental plane with the $\widehat{W}$ axis being the rotational axis of the orbiting body defined by:

$$
\widehat{W}=\frac{\vec{r}_{1} \times \vec{r}_{2}}{\left|\vec{r}_{1}\right|\left|\vec{r}_{2}\right|}
$$


Using this coordinate system, which is ideal for observing orbital perturbations, the initial slant-range for the third observation can be determined through Eq.2.15.

$$
\rho_{3}=\frac{-\vec{r}_{\text {site }_{3}} \cdot \widehat{W}}{\widehat{L}_{3} \cdot \widehat{W}}
$$

Again Eq.2.14 can be used with the new slant-range in order to acquire the initial $\vec{r}_{3}$ vector. Now that all the initial position values are calculated, Eq.2.16 and Eq.2.17 are iterated through for $\mathrm{j}=2,3$ and $\mathrm{k}=1,2$ to determine the trigonometric components of the difference in the angle between the respective positions. The orbital motion is denoted as $t_{m}$ where prograde motion is defined as (+1) and retrograde motion is defined as (-1).

$$
\begin{gathered}
\cos \left(\theta_{j}-\theta_{k}\right)=\frac{\vec{r}_{j} \cdot \vec{r}_{k}}{\vec{r}_{j} \vec{r}_{k}} \\
\sin \left(\theta_{j}-\theta_{k}\right)=t_{m} \sqrt{1-\cos ^{2}\left(\theta_{j}-\theta_{k}\right)}
\end{gathered}
$$

Once the angular quantities between all positions are determined, the constants $c_{1}$, $c_{3}$, and $p$ can be found. These values depend on if the angle between the first and third observation point is over or under $180^{\circ}$ and are used to determine the mean anomaly of the positions later on. Due to the fact that all of the observations have an angular distance less than $180^{\circ}$, Eq.2.18-2.20 will solely be used for these values.

$$
\begin{aligned}
& c_{1}=\frac{r_{2} \sin \left(\theta_{3}-\theta_{2}\right)}{r_{1} \sin \left(\theta_{3}-\theta_{1}\right)} \\
& c_{3}=\frac{r_{2} \sin \left(\theta_{2}-\theta_{1}\right)}{r_{3} \sin \left(\theta_{3}-\theta_{1}\right)} \\
& p=\frac{c_{3} r_{3}-c_{1} r_{2}+r_{1}}{-c_{1}+c_{3}+1}
\end{aligned}
$$


With these constants, the cosine and sine values of the eccentricity of the orbit are found in order to ultimately determine the associated eccentricity of the observed object through the use of Eq.2.21-2.23.

$$
\begin{gathered}
e \cos \left(\theta_{i}\right)=\frac{p}{r_{i}}-1 \quad i=1,2,3 \\
e \sin \left(\theta_{2}\right)=\frac{-\cos \left(\theta_{2}-\theta_{1}\right) e \cos \left(\theta_{2}\right)+e \cos \left(\theta_{1}\right)}{\sin \left(\theta_{2}-\theta_{1}\right)} \\
e=\sqrt{\left(e \cos \left(\theta_{2}\right)\right)^{2}+\left(e \sin \left(\theta_{2}\right)\right)^{2}}
\end{gathered}
$$

The acquisition of a preliminary eccentricity value for the orbit then leads to the calculation of the rest of the COEs of the orbit and ultimately to the eccentric anomaly and mean anomaly between the first and third observation to the central position. The mean anomaly between each of the positions are then divided by the mean motion, $n$, and subtracted from the time difference between each observation in relation to the central position, $\tau$, seen in Eq.2.24 and Eq.2.25.

$$
\begin{aligned}
& F_{1}=\tau_{1}-\frac{\Delta M_{12}}{n} \\
& F_{2}=\tau_{3}-\frac{\Delta M_{32}}{n}
\end{aligned}
$$

The norm of the sum of these two values are used to estimate the accuracy of each pass. The goal is now to diminish the $F_{1}$ and $F_{2}$ values to zero in order to maximize the accuracy of the final iteration. 
These calculations are repeated for $F_{1}\left(r_{1}+\Delta r_{1}, r_{2}\right)$ and $F_{2}\left(r_{1}, r_{2}+\Delta r_{2}\right)$ using finite differencing of the positions. By setting $\Delta r_{1}$ and $\Delta r_{2}$ equal to a small fraction of the actual value, Eq.2.26 and Eq.2.27 can be used to step forward in time.

$$
\begin{aligned}
& \frac{\partial F_{i}}{\partial r_{1}}=\frac{F_{i}\left(r_{1}+\Delta r_{1}, r_{2}\right)-F_{i}\left(r_{1}, r_{2}\right)}{\Delta r_{1}} \\
& \frac{\partial F_{i}}{\partial r_{2}}=\frac{F_{i}\left(r_{1}, r_{2}+\Delta r_{2}\right)-F_{i}\left(r_{1}, r_{2}\right)}{\Delta r_{2}}
\end{aligned}
$$

This process continues until convergence is reached to within the desired tolerance and the final desired COE values are obtained. Using celestial mechanics and the Lagrange coefficients previously mentioned in $§ 2.2 .1$ (denoted below as $\mathrm{f}$ and $\mathrm{g}$ ), the velocity and position at the central imaged location can be acquired with Eq.2.28-2.30.

$$
\begin{gathered}
f=1-\frac{a}{r_{2}}\left(1-\cos \left(\Delta E_{32}\right)\right) \\
g=\tau_{3}-\sqrt{\frac{a^{3}}{\mu}}\left(\Delta E_{32}-\sin \left(\Delta E_{32}\right)\right) \\
\overrightarrow{v_{2}}=\frac{\overrightarrow{r_{3}}-f \overrightarrow{r_{2}}}{g}
\end{gathered}
$$

\subsection{Orbit Determination and Estimation}

Orbital determination is the prediction of an orbital path by propagating a solution of subsequent observations based off of the IOD values previously calculated. The ability to accurately predict an object's position and velocity including realistic measures of uncertainty is a complex problem. The first estimation methods used in orbital 
determination were first created seemingly independently by both Karl Gauss and Adrian Legendre in the early $19^{\text {th }}$ century depicting the process of least squares. In summation of the least squares process, Gauss stated, "The most probable value of the unknown quantities will be that in which the sum of the squares of the differences between the actually observed and computed values multiplied by numbers that measure the degree of precision is a minimum." This minimum value is statistically the best value to the true location of the object given the collected data [22].

Throughout the $20^{\text {th }}$ century, there were steady improvements in non-Gaussian error statistics which eventually led to the publication of A New Approach to Linear Filtering and Prediction Problems by Rudolf Kalman (1960) introducing the method of Kalman Filtering [27]. The major difference in these two approaches is that the Kalman Filter continuously updates the epoch time, thus estimating the state at each successive epoch. Secondly, the Kalman Filter carries all information concerning past measurements in its current state and covariance estimations. These OD techniques are extensively covered in the text Statistical Orbit Determination by Tapley, Schutz, and Born but will be briefly summarized in the following section [22].

Since orbital trajectories are non-linear, the least squares method and Kalman Filter alone are not particularly accurate enough to successfully predict the future positions of the observed objects in space. Both these methods can be modified to account for the nonlinear systems. The Least Squares Method implements the use of differential corrections while the Kalman Filter can be modified into a process called the Extended Kalman Filter (EKF) which will be highlighted in $\$ 2.3 .2$. 


\subsubsection{Non-Linear Least Squares}

The Least Squares Method (LS) is a technique used to estimate the best fit solution to a linear system. By having a general understanding of the dynamics of the system in question, a computed solution can be generated based on the independent variable, as depicted in Eq.2.31.

$$
y_{c_{i}}=\alpha+\beta x_{o_{i}}
$$

With the computed solution of the system defined, the residual of the system can be defined as the difference of the observed and calculated data points seen in Eq.2.32.

$$
\vec{r}_{i}=y_{o_{i}}-y_{c_{i}}
$$

The least-squares cost function, seen in Eq.2.33, is the summation of the residual values for all data points and a minimum of the function. A squared function is used since a parabola $\left(\vec{r}_{i}^{2}\right)$ has a minimum whereas a line $\left(\vec{r}_{i}\right)$ does not.

$$
J=\sum_{i=1}^{N} \vec{r}_{i}^{2}
$$

This linear least-squares approach can also be used in non-linear systems. Nonlinear systems can be linearized in order to obtain an approximate solution, and then iterated upon to refine the answer to a minimum solution. The differential correction technique can accurately estimate the state vector of an object from optical measurements.

The optical images gathered act as the observed values and can be depicted as a state vector as shown below with variables of slant range, azimuth, and elevation of the object. 


$$
y_{o_{i}}=\left[\begin{array}{c}
\rho_{o} \\
a z_{o} \\
e l_{o}
\end{array}\right] \text { at times }=t_{i}
$$

The computed value needed to produce the required residuals to determine estimated orbits are derived from the IOD orbit predicted is depicted as:

$$
y_{c_{i}}=\left[\begin{array}{c}
\rho_{c} \\
a z_{c} \\
e l_{c}
\end{array}\right] \text { at times }=t_{i}
$$

Because the predicted orbit path is a non-linear function, a Taylor series expansion is used in approximation of a nominal trajectory. When ignoring the higher order terms, this vector can be expressed as:

$$
y_{c_{i}}=y_{n_{i}}+\Delta r_{I} \frac{\partial y_{n_{i}}}{\partial r_{I}}+\Delta r_{J} \frac{\partial y_{n_{i}}}{\partial r_{J}}+\Delta r_{K} \frac{\partial y_{n_{i}}}{\partial r_{K}}+\Delta v_{I} \frac{\partial y_{n_{i}}}{\partial v_{I}}+\Delta v_{J} \frac{\partial y_{n_{i}}}{\partial v_{J}}+\Delta v_{K} \frac{\partial y_{n_{i}}}{\partial v_{K}}
$$

The nominal trajectory is a function of a nominal vector at each observation time and is defined as:

$$
y_{n_{i}}=f\left(r_{I} r_{J} r_{K}, v_{I} v_{J} v_{K}, t_{i}\right)
$$

This new $y_{c_{i}}$ value can then be input into Eq. 2.32 in order to determine the residuals of the system. A weighting value, $w_{i}$, must first be added to minimize the cost function. The weighting value for each measurement parameter is the reciprocal of the appropriate standard deviation of the associated error. Similarly to Eq.2.33, the sum of the squares of the residuals is used, but now dealing with residual matrices and weightings, the cost function is transformed into Eq.2.34.

$$
J=\sum_{i=1}^{N}\left(w_{i} \vec{r}_{i}\right)^{T}\left(w_{i} \vec{r}_{i}\right)
$$

Through matrix algebra, this cost function results in a partial-derivative matrix, A. 
This matrix is defined as the partial derivative of the observation measurements, $\rho, a z$, and $e l$, with respect to the state space, which are the three positional vectors and three velocity vectors defining the data point.

$$
A=\frac{\partial o b s e r v a t i o n s}{\partial X_{o}}=\left[\begin{array}{llllll}
\frac{\partial \rho_{i}}{\partial r_{I_{o}}} & \frac{\partial \rho_{i}}{\partial r_{J_{o}}} & \frac{\partial \rho_{i}}{\partial r_{K_{o}}} & \frac{\partial \rho_{i}}{\partial v_{I_{o}}} & \frac{\partial \rho_{i}}{\partial v_{J_{o}}} & \frac{\partial \rho_{i}}{\partial v_{K_{o}}} \\
\frac{\partial a z_{i}}{\partial r_{I_{o}}} & \frac{\partial a z_{i}}{\partial r_{J_{o}}} & \frac{\partial a z_{i}}{\partial r_{K_{o}}} & \frac{\partial a z_{i}}{\partial v_{I_{o}}} & \frac{\partial a z_{i}}{\partial v_{J_{o}}} & \frac{\partial a z_{i}}{\partial v_{K_{o}}} \\
\frac{\partial e l_{i}}{\partial r_{I_{o}}} & \frac{\partial e l_{i}}{\partial r_{J_{o}}} & \frac{\partial e l_{i}}{\partial r_{K_{o}}} & \frac{\partial e l_{i}}{\partial v_{I_{o}}} & \frac{\partial e l_{i}}{\partial v_{J_{o}}} & \frac{\partial e l_{i}}{\partial v_{K_{o}}}
\end{array}\right]
$$

This matrix defines the relation of the observations at many different times to a state vector at a specific epoch and describes how changes in the initial state, $X_{o}$, affect the computed observation measurements.

The weighting matrix, $W$, is based off of the noise statistics and biases inherent in the observation system being used, expressed below:

$$
W=\left[\begin{array}{ccc}
\frac{1}{\sigma_{\rho}^{2}} & 0 & 0 \\
0 & \frac{1}{\sigma_{a z}^{2}} & 0 \\
0 & 0 & \frac{1}{\sigma_{e l}^{2}}
\end{array}\right]
$$

The $\tilde{b}$ matrix is the culmination of the residual values of each observation parameter between the nominal and observed values.

$$
\tilde{b}=\left[\begin{array}{c}
\rho_{o i}-\rho_{n i} \\
a z_{o i}-a z_{n i} \\
e l_{o i}-e l_{n i}
\end{array}\right]
$$


Using these three matrices, the values of $A^{T} W A$ and $A^{T} W \tilde{b}$ are accumulated in order to determine the update value to the state with Eq.2.35.

$$
\delta \hat{x}=\left(A^{T} W A\right)^{-1} A^{T} W \tilde{b}=\mathrm{P} A^{T} W \tilde{b}
$$

The covariance matrix of the system is shown above as matrix $P$ and the nominal state becomes:

$$
X_{n o m}=X_{n o m}+\delta \hat{x}
$$

This process is continued until the iteration converges onto a solution of the nominal state vector and an associated orbital path.

Figure 2.7 illustrates an example of the generated modified orbits in relation to the nominal orbit and true orbit. We find the partial derivative of the observations with respect to the state by modifying the initial state, propagating the nominal and modified states to each observation time and finding the difference of the observations.

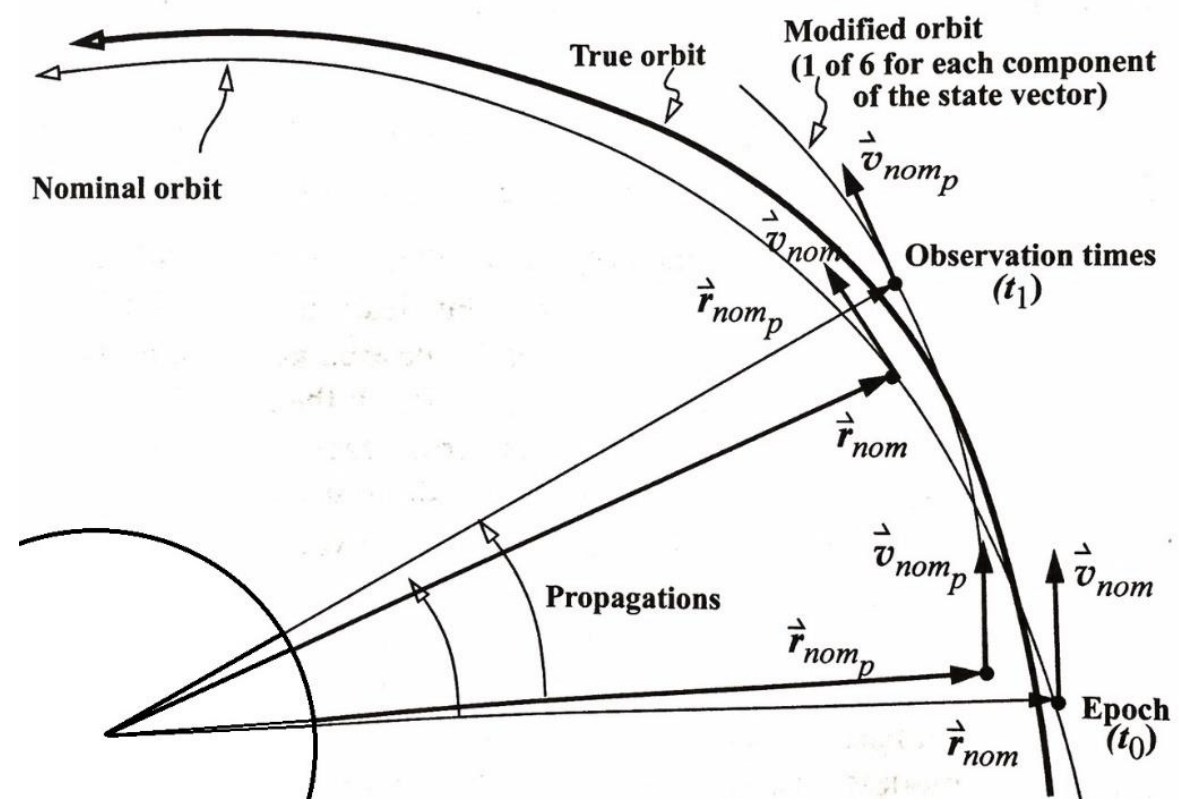

Figure 2.9: Least Squared Finite Differencing [32] 
This figure shows movement of one nominal and modified state vector to an observed time, where nom ${ }_{p}$ are the modified orbit values of one of the partial derivative state vectors.

The least-squares method minimizes a cost function that depends on both computed and observed values which is dependent on the dynamics and the initial state of the system. The nominal orbital path will ultimately be determined by iterating upon the modified orbits to the local minimum.

\subsubsection{Extended Kalman Filtering}

The Kalman filter is a technique for computing the best estimate of the state of a time-varying process. Since Kalman filtering is used for linear systems and satellite orbits are nonlinear, we must use the EKF process to account for the system dynamics. EKF uses current state estimations to determine a new state estimate at each observation time using a predictor/corrector iteration system. Seen in Fig. 2.8, the EKF process predicts a solution, $\bar{X}_{i}$, for a future position based off of the past data, and iterates upon itself to eventually converge on the best fit orbit.

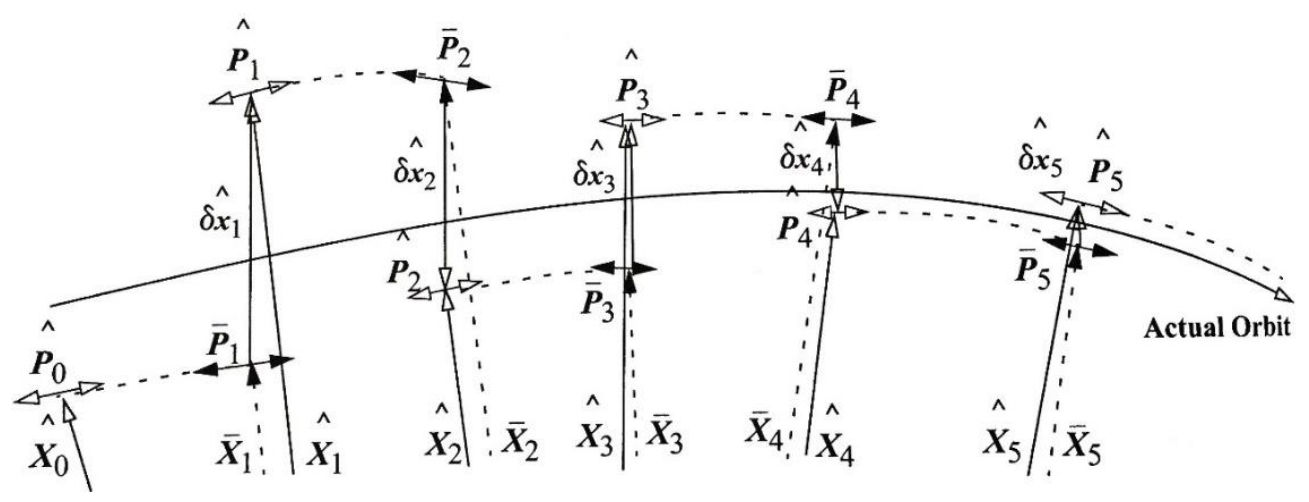

Figure 2.10: Extended Kalman Filtering Method [32] 
The nonlinear dynamics of this problem must first be linearized, creating the error state transition matrix, $\Phi$. By linearizing the equations of motion, a simplified approximation can be developed to compute the differences over a small time interval with the appropriate system dynamics. Similar to the Least Squares Method, a partial derivative matrix, $A$, relating the observed parameters and the state must be established. A key difference that can be noted between the two methods is that the state is refined through iteration and the partial derivate matrix is updated with the new state estimate at each step:

$$
A_{k+1}=\frac{\text { dobservations }}{\partial \widehat{X}_{k+1}}
$$

With the initial state $\left(\hat{X}_{k}\right)$ already defined, subsequent steps can be propagated forward using the state transition matrix to determine the error covariance matrix $\left(\widehat{P}_{k}\right)$ using the second moment of the process-noise, $Q$, and the previous error covariance shown in Eq.2.36.

$$
\bar{P}_{k+1}=\Phi \widehat{\mathrm{P}}_{k} \Phi^{T}+Q
$$

The process-noise matrix is the variance of the observation parameters due to the inherent error within the system being used. Once the error covariance matrix and state are predicted for the next time step, these values are used to update the new state estimate.

In order to determine the state error estimate, $\delta \hat{x}_{k+1}$, the Kalman gain needs to be updated for each step as well. The Kalman gain, $\mathrm{K}$, is determined through the current steps error covariance and partial derivative matrix and multiplied with the residual values, $\tilde{b}$, to get the current state error estimate. This value can then be added to the previously predicted step to get the new updated value shown in Eq.2.37. 


$$
\hat{X}_{k+1}=\bar{X}_{k+1}+\delta \hat{x}_{k+1}
$$

Using the same Kalman gain and partial derivative matrix, the updated covariance matrix becomes Eq.2.38.

$$
\widehat{P}_{k+1}=\bar{P}_{k+1}-K_{k+1} A_{k+1} \bar{P}_{k+1}
$$

With these updated values, the process continues to iterate upon itself using the previous estimate of the state and error covariance in each subsequent update until it converges on the minimum orbital path. 


\section{INSTRUMENTATION AND PROCEDURE}

\subsection{Instrumentation}

\subsubsection{Observatory}

The Cal Poly observatory is located in the center of the Cal Poly campus in San Luis Obispo, California. The longitude and latitude coordinates of the observatory are $35.30^{\circ}$ $\mathrm{N}, 120.66^{\circ} \mathrm{W}$ at an altitude of $105.8 \mathrm{~m}$ above sea level. Due to the observatories location in the center of campus and low standing in relation to its surrounding buildings, there is a fairly considerable amount of light pollution. The observational equipment is fitted with a $12^{\prime}$ diameter dome in order to minimize the negative effects of the surrounding light.

The dome has a lower observational limit of $25^{\circ}$ above the horizon due to the previously mentioned surrounding buildings and trees. The dome is equipped with an automated tracking system calibrated in accordance with the telescope's pointing direction. 


\subsubsection{Telescope}

The Meade LX600-ACF 14" telescope acted as the main optical device used for all observations and can be seen in Fig. 3.1. The telescope is an Advanced Coma Free (ACF) optical design and mounted on a double-tine fork mount. Although the telescope is equipped with a Starlock ${ }^{\mathrm{TM}}$ system, it was disabled before each set of observations since it was experiencing accuracy issues and not vital in the imaging process. The telescope has an upper observable limitation of $\sim 75^{\circ}$ above the horizon due to physical constraints imposed by the mounting system geometry. Due to the relatively small aperture size and pricing of $\$ 7,999.00$, this telescope is considered to be "amateur-sized", with a cost and portability thought to be reasonable for the more serious amateur astronomer.

The telescope is fitted with an $f / 6.3$ focal reducer creating a larger FOV of roughly $22^{\prime} \mathrm{X} 15^{\prime}$ arcminutes as well as increases the brightness of the images taken through the apparent focal length.

The telescope and focal reducer used by Schmalzel were the Meade LX200 12" telescope and the $\mathrm{f} / 3.3$ focal reducer. The increased diameter of the Meade LX600 14" allows for a larger photon intake enhancing the ability to detect objects with fainter apparent magnitudes. The reasoning behind the use of the $\mathrm{f} / 6.3$ focal reducer as opposed to the $f / 3.3$ was that although the $f / 3.3$ reduction would increase the FOV and brightness of the image, it would hinder the resolution. Since A $2 X 2$ binning scheme was already diminishing the resolution (reasoning discussed in §3.2.3), the additional loss did not seem justified for a marginal gain in observable area [11]. 


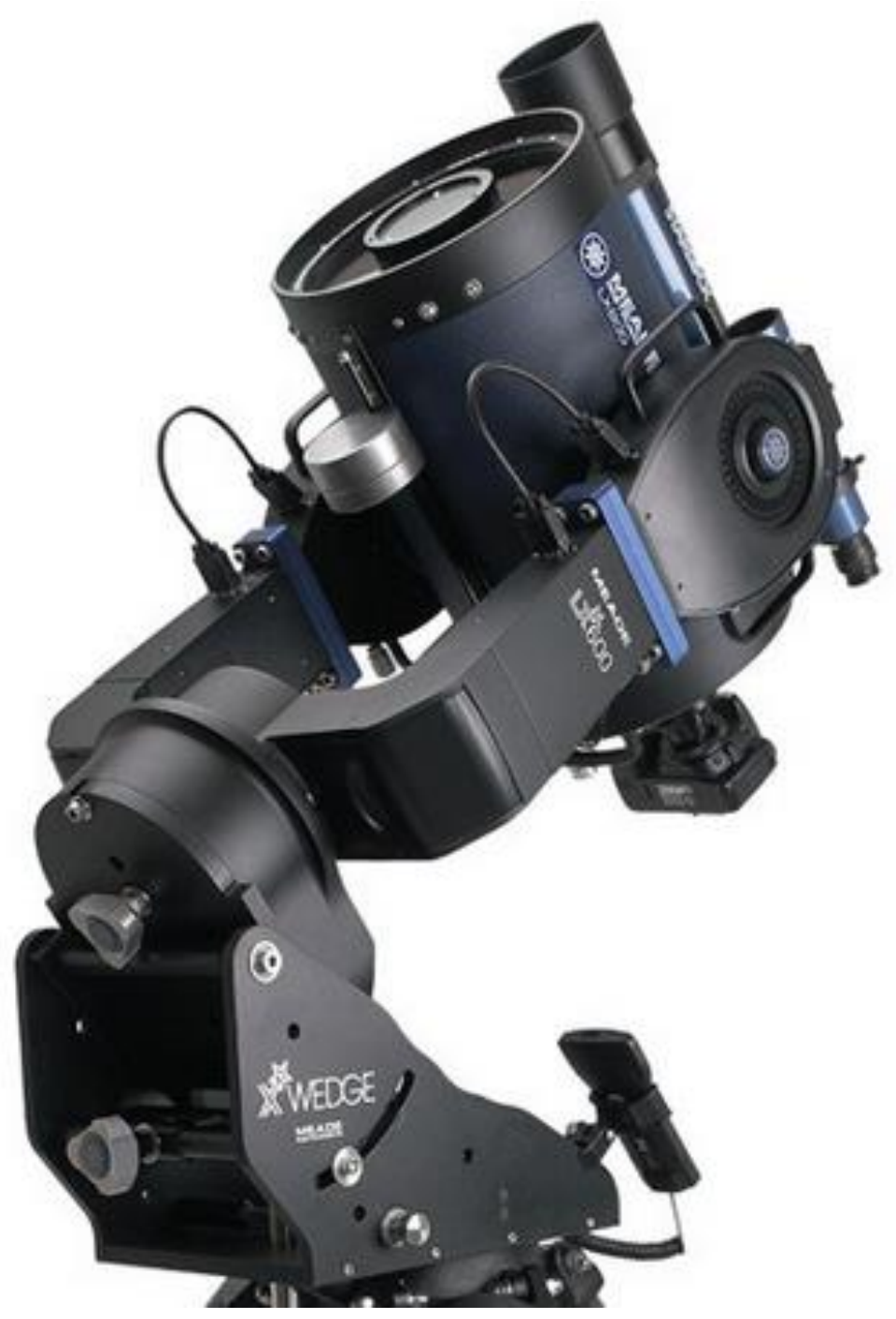

Figure 3.1: Meade LX600-ACF 14" Telescope

Table 3.1: Meade LX600-ACF 14" Telescope Specifications

\begin{tabular}{|c|c|}
\hline Specification & Quantity \\
\hline Aperture & $355 \mathrm{~mm}$ \\
\hline Optical Design & Advanced Coma Free (ACF) \\
\hline Focal Length & $\mathrm{f} / 8$ \\
\hline Focal Ratio & 0.326 arc-seconds \\
\hline Resolving Power & $\mathrm{f} / 6.3$ \\
\hline Focal reducer & 1 arc-minute $(+/-)$ \\
\hline Pointing Precision & \\
\hline
\end{tabular}




\subsubsection{CCD Camera}

The SBIG ST-10XME CCD Camera with CFW-8A filter wheel attachment was used to capture the incoming light. The CCD is attached to the eyepiece behind the focal reducer in order to capture and digitally transmit photons funneled through the main optics to be analyzed using TheSkyX astronomical software. Although the CCD is equipped with multiple filter options, the "clear" filter was consistently used in order to maximize the number of photons captured and not to eliminate any wavelengths through a colored filter.

This CCD camera was used in replacement of the Lumenera SKYnyx2-0 monochrome astrophotography camera used to record telescopic instruments in Brock Schmalzel's thesis [19]. The reason for the alteration in recording instrumentation is due to the reduced read noise generated and dramatically increased total pixel count [8][21]. Both of these quantities allow for an increased resolution of the images taken and are especially useful when determining orbital paths to a higher degree of accuracy considering instrumentation limitations. 


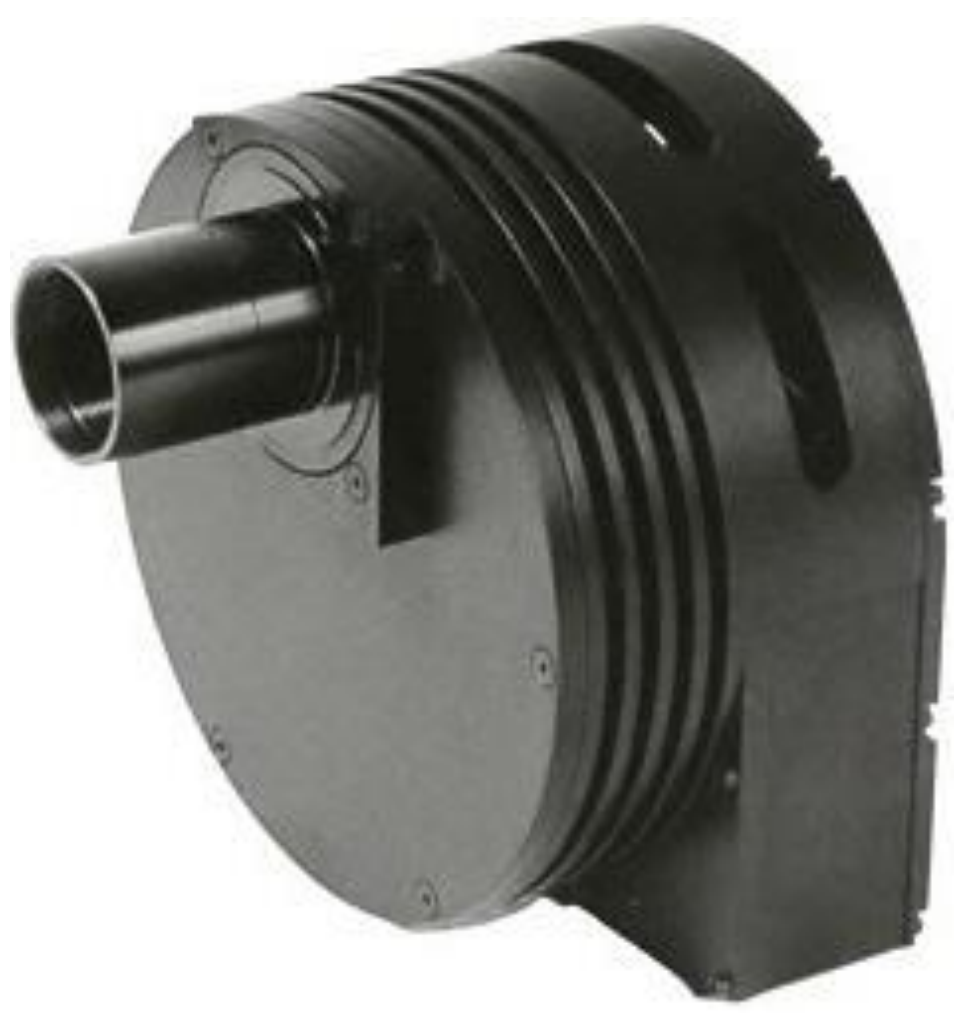

Figure 3.2: SBIG ST-10XME CCD Camera

Table 3.2: SBIG ST-10XME CCD Camera Specifications

\begin{tabular}{|c|c|}
\hline Specification & Quantity \\
\hline Pixel Array & $2184 \times 1472$ pixels \\
\hline Total Pixels & 3.2 million \\
\hline CCD Size & $14.9 \times 10 \mathrm{~mm}$ \\
\hline Full Well Capacity & $\sim 77,000 \mathrm{e}-$ \\
\hline Read Noise & $8.8 \mathrm{e}$ RMS \\
\hline Full Frame Download & $\sim 8.7 \mathrm{sec}$ \\
\hline Filter Wheel & CFW-8A \\
\hline
\end{tabular}




\subsubsection{Focuser}

The Optec TCF-S focuser ensures the optical accuracy of the images. The telescope was focused on a star before each night of observation in order to narrow the Gaussian distribution of light. As seen in Fig.3.3, There are four graphs indicating the precision in which the CCD receiver is set to the effective focal length. The collected photons are broadened on the CCD pixel array from atmospheric effects and telescope optics. Any variation from the telescopes focal point will generate a larger Half Flux Diameter (HFD).

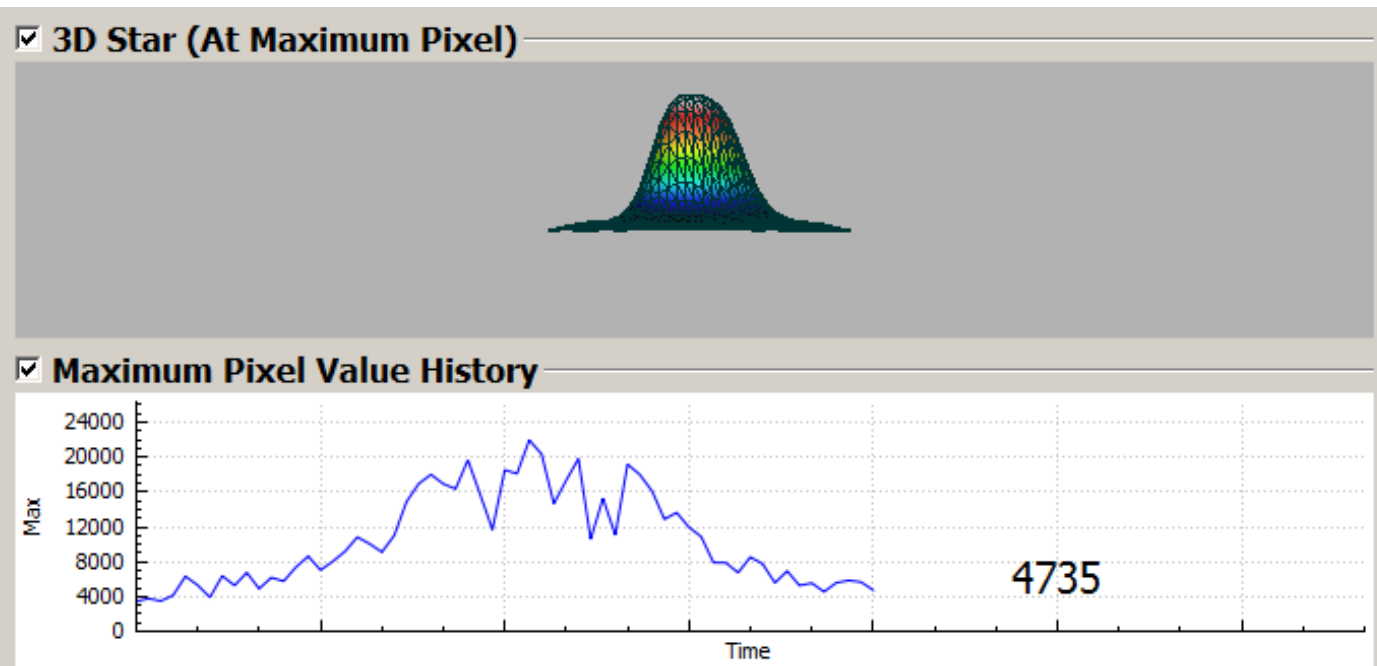

\section{Half Flux Diameter (HFD) History}

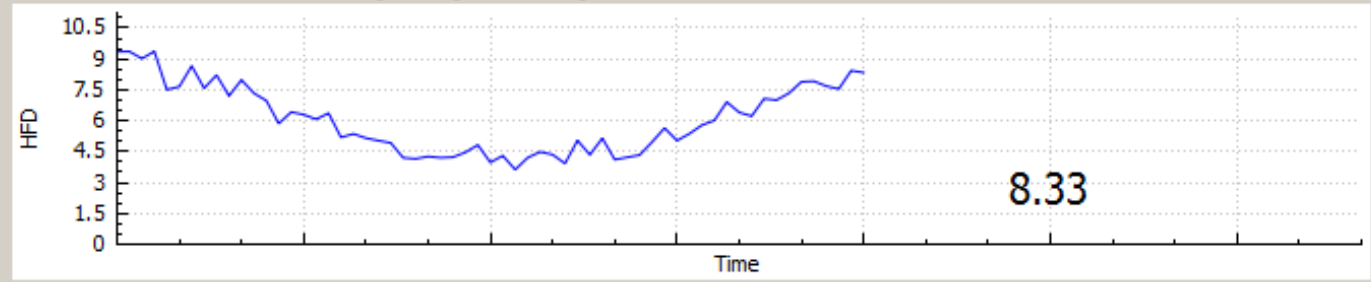

\section{Focus V-Curve (HFD vs Focuser Position)}

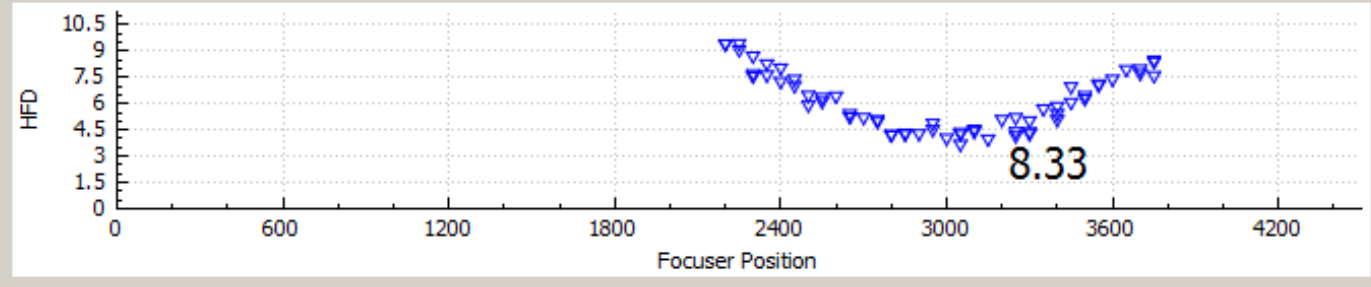

Fig 3.3: HFD Calibration 
The top graph is a 3D rendering of the Gaussian photon dispersion around the pixel closest to the full well capacity. The goal is to minimize the dispersion of the photons so that they are more accurately focused on to less pixels creating brighter and sharper image. The second and third graph are running measures of the max pixel value and HFD. It is apparent that as the HFD shrinks, the max pixel value will increase.

To ensure the sharpest and brightest image, a continuous series of images are taken while the focuser is altered in increments of 50 steps after each image. Once a trend is established defining a minimum on the Focus V-Curve, the step count is set to ensure the brightest image resolution.

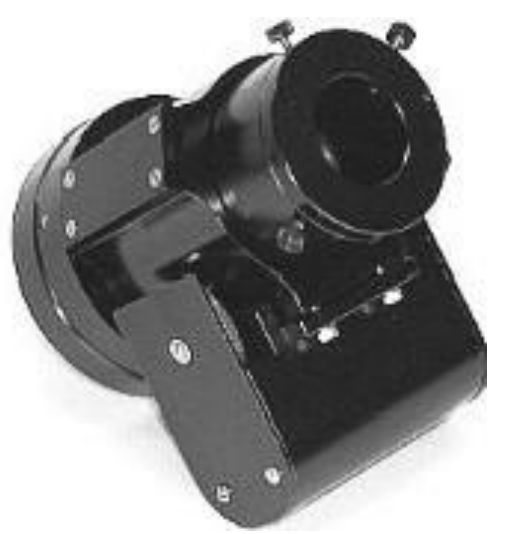

Figure 3.4: Optec TCF-S Focuser

Table 3.3: Optec TCF-S Focuser Specifications

\begin{tabular}{|c|c|}
\hline Specification & Quantity \\
\hline Step Resolution & 2.2 microns \\
\hline Number of Steps & 7,000 \\
\hline Focuser Travel & $0.6 ”(15.2 \mathrm{~mm})$ \\
\hline
\end{tabular}




\subsubsection{Software}

MATLAB R2015b was the technical computing software used for running the orbital determination algorithms as well as orbital error analysis. The astronomical software used to orient the telescope and dome, display and catalog the CCD images, and calibrate the focuser was TheSkyX software version 10.3 [24].

The .FIT file format scientific images were viewed with SAOImage ds9 software which allows each image to be scaled, magnified, determine points of interest down to individual pixels, and view the header file of each image providing the time stamps, pointing coordinates, and more. The values provided by the file headers as well as the pixel count for comparison stars and satellite locations were input into Microsoft Excel sheets and saved as .csv files to transfer to MATLAB as the usable raw data.

Before observation nights, satellite pass times and solar phase angles were checked using the Systems Tool Kit (STK) software which also provided the predicted azimuth, elevation and range (AER) values for the times of each observation later used in the MATLAB raw data files.

The final software system used was the astronomical image database Aladin v9.0 Sky Atlas which was used to both verify the pointing accuracy of the telescope as well as determining the comparison star's RA and DEC within the FOV of each image during analysis. 


\subsection{Procedure}

\subsubsection{Procedural Overview}

The overall procedural flowchart can be seen below in Fig.3.5. The first step in taking an observation is to acquire a recent TLE from either an online external database or outside source. Due to the small FOV of the Cal Poly telescope in relation to larger observatories around the world, it would take much longer to acquire the initial passing satellite's path independently. Externally acquired TLEs are initially used in order to specify the general area that will be observed for the first pass of the specific orbit. Once the desired satellite TLEs are chosen, they are analyzed and inputted into the observational software to propagate the satellite's position forward in time to an observable location based on their orbital parameters and relative location to the point of observation.

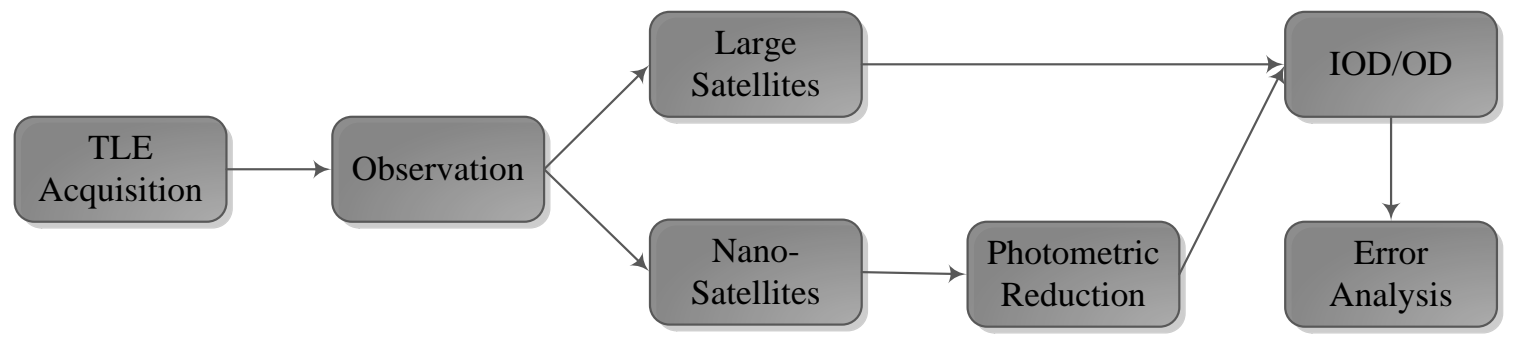

Figure 3.5: Procedural Flowchart

The two types of satellites attempting to be observed differ drastically in size, denoted as "large satellites" and "nanosatellites" as seen in Fig.3.5. Since the nanosatellites have a drastically decreased reflective surface area, they are much more difficult to image. The larger satellite observations do not require photometric reduction to locate the defined path of the satellites within the FOV, but would be useful in nanosatellite images to separate the faint orbital paths from the background noise. All images are then run through the 
orbital determination methods previously mentioned in Chapter 2 as well as undergo an error analysis to determine any underlying trends in data inaccuracies revealing possible causes.

An overview of the data flow through the various software programs and databases used in the preparation and observation stages can be seen below in Fig.3.6.

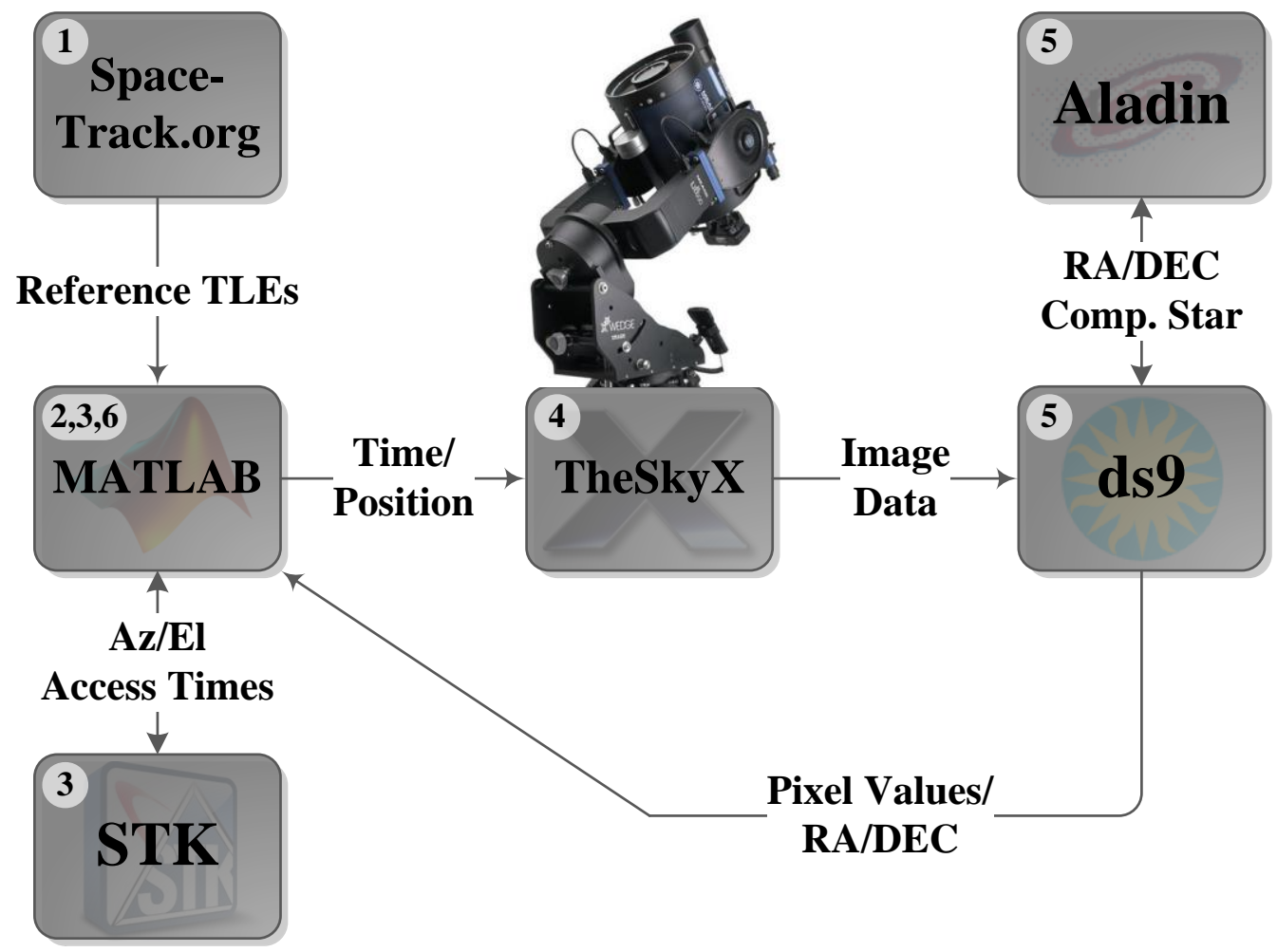

Figure 3.6: Data Flow Overview

The flow of data originates at the stage labeled " 1 " and moves through sequentially, while stages showing matching numbers require data flow in both directions and occur concurrently. These procedural stages will be explained in the upcoming sections. 


\subsubsection{Preparation}

Before any observation takes place, the required times and positions of the selected satellites for each night must first be collected. The most recent TLEs of orbiting satellites were taken from the Space-track.org online database and served as the initial values for observation. The Globalstar satellite system TLEs were used exclusively for the "large satellite" observations due to their cross sectional area, reflectivity, upper LEO altitude, and the large quantity of possible targets [9]. These factors allowed for a high contrast from the background noise as well as the possibility for multiple observations of multiple targets in one night.

The full set of Globalstar and CubeSats being tracked and updated in the Spacetrack database were transferred into a text file on the day leading to each night of observation. In addition to these publicly accessible TLE files, the nanosatellite TLEs of five additional 3U CubeSats with the Satellite IDs of BRICSAT-P, PSAT, ULTRASat10, ULTRASat4, and ULTRASat5 were provided by Justin Foley [8]. Figure 3.7 illustrated the relative size of the Globalstar satellites to the Cubesats. The average surface area of the Globalstar satellites are roughly $18.3 \mathrm{~m}^{2}$ while the Cubesats with the largest surface area (FLOCK Cubesats) are roughly $0.21 \mathrm{~m}^{2}$. This nearly $90: 1$ ratio in size difference depicts the dramatically dampened apparent magnitude of the satellite and the hindered ability to observe during transit. Even though the magnitude of the nano-satellites are much dimmer than the larger satellites, the determined apparent magnitude still lies within the boundaries of the limiting magnitude of the LX-600 14" "telescope. 

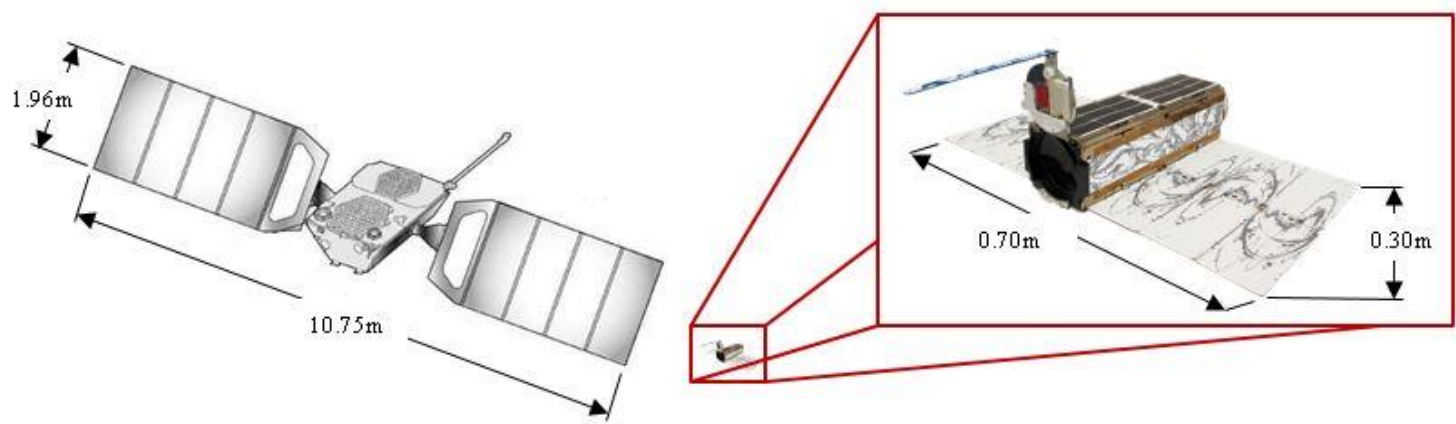

Figure 3.7: Satellite Relative Size and Dimensions

Although attempts were made in observing the additional CubeSats, none were successful due to the minimal elevation requirements at the time of observation that are covered more thoroughly in Ch.5.

The TLEs for each satellite being observed on a particular night were input into an STK scenario to determine access times, elevation angles, and illumination periods. The TLE Azimuth-Elevation-Range (AER) reports were generated for a given observatorybounded sensor using the Simplified General Perturbation (SGP4) propagator implemented within STK. The sensor was modeled as a Simple Conic type with an $70^{\circ}$ half cone angle to simulate the relative viewing angle of the observatory.

Seen below in Fig.3.7 is a generated image of multiple FLOCK CubeSats with the observable transit areas highlighted in cyan. The access times for all passes over the course of 24 hours are cross referenced with illumination times of each satellite during the corresponding pass. 


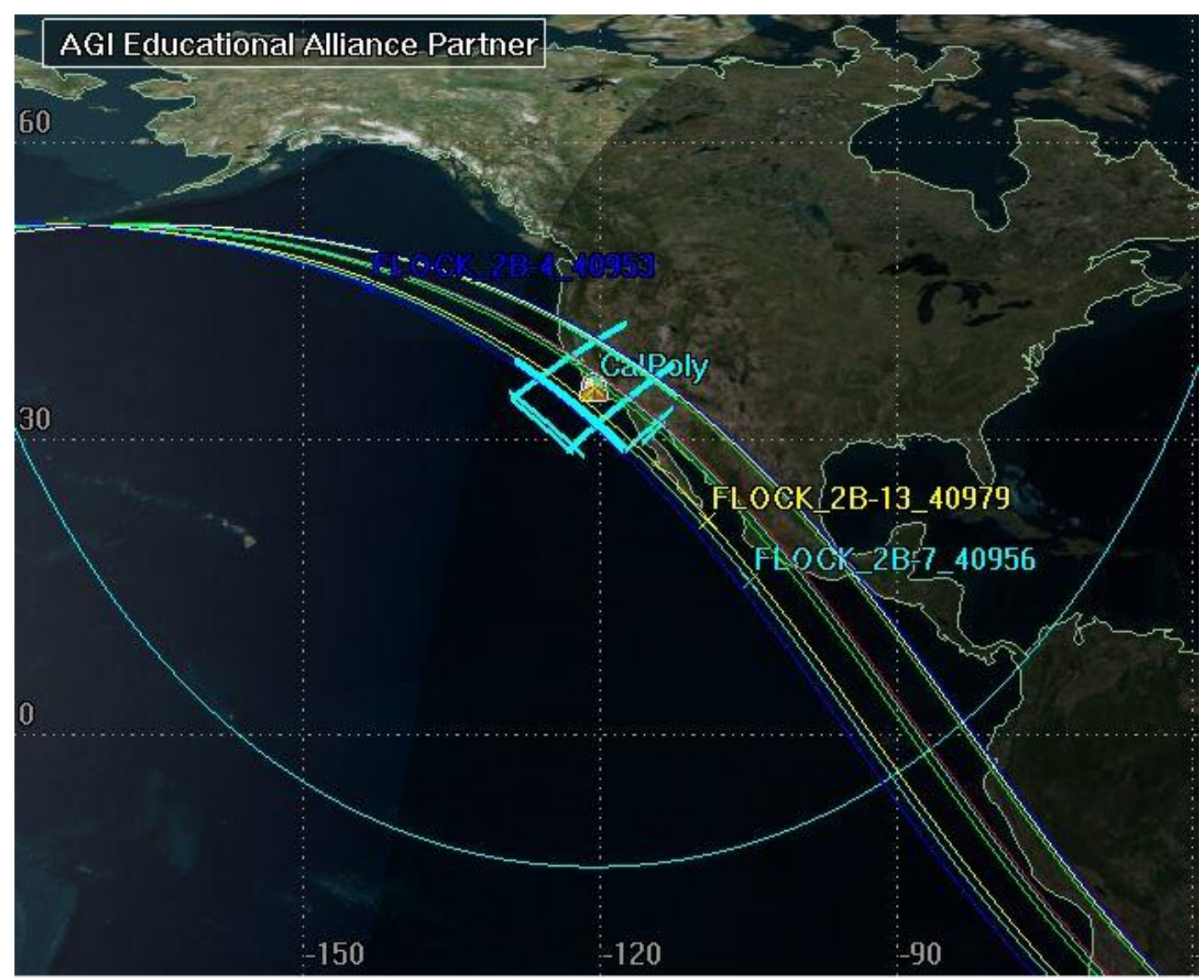

Figure 3.8: STK Access and Illumination Times

Once the access times and illumination times were confirmed, a quick elevation check using the STK's AER reports for each pass verified a suitable distance above the horizon and surrounding buildings. Seen in Fig.3.8, based on the location of the Sun (top left corner) and the angle of the pass relative to the point of observation, the associated SPA is very important to consider. This not only applies to the Globalstar satellites, but also to the FLOCK Cubesats in which have deployable solar panels that require pointing as well. 


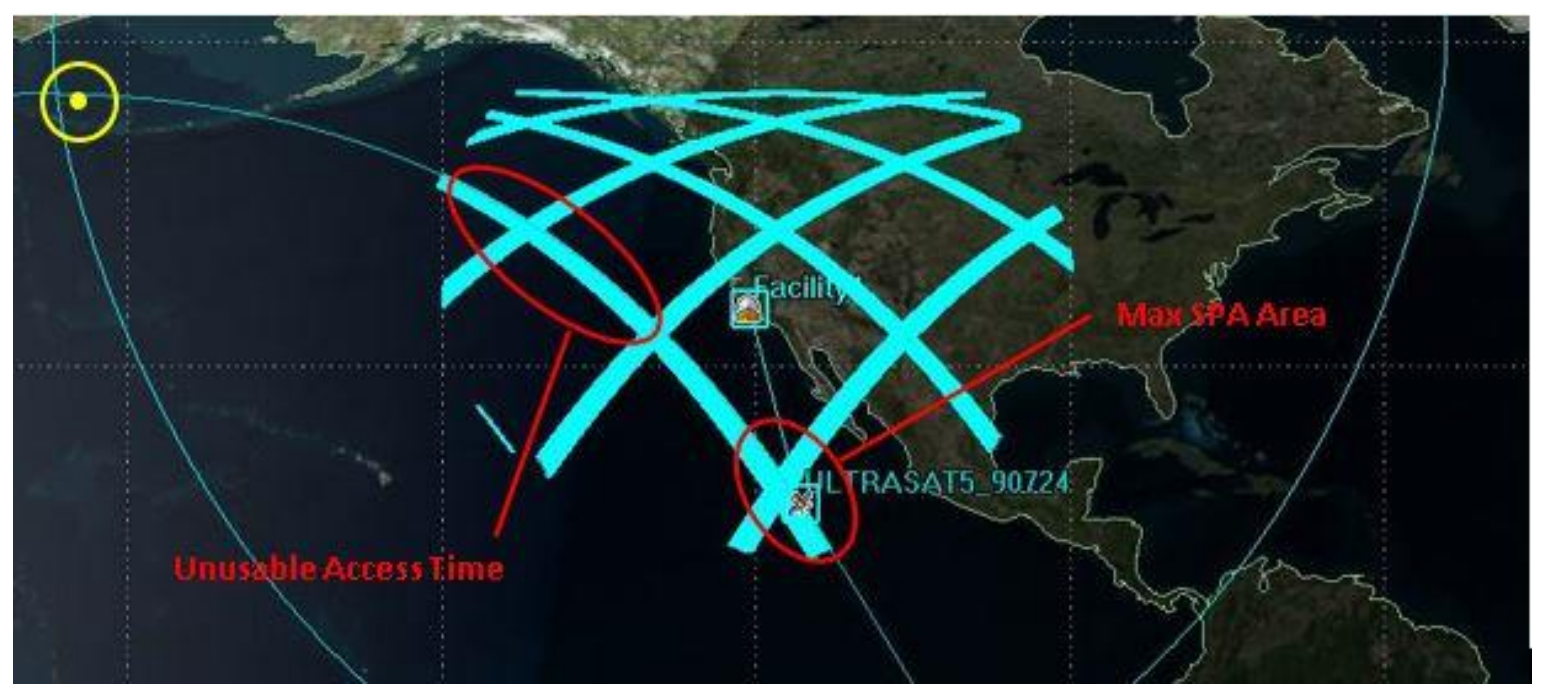

Figure 3.9: Determination of Imaging Times

Due to the relatively high surface area of satellite's solar panels being oriented toward the Sun, the maximum surface area must be at a SPA past the observation point in order to detect any reflected light which renders the entire first half of the access time unusable. The reflective surface area increases as it approaches the end of the visible period and enters the Earth's penumbra. The AER reports generated in STK were exported in a .csv format file and processed using MATLAB in order to determine acceptable SPA times. With the SPA and extremely low orbits of the CubeSats to consider, the overlap of access time and the illumination period were very short. However, based off of these constraints, the theoretical optimal observation times were determined for each pass. 


\subsubsection{Observation}

Once at the observatory, a series of precautionary steps were taken in order to ensure proper system functionality and preserve the instrumentation. Power to the dome, focuser, CCD camera, and the on-site terminal. Once the terminal is activated and TheSkyX software is booted-up, the dome, focuser, and CCD camera are electronically connected to the terminal. The telescope protective covering and lens caps are removed after the dome is fully opened to minimize any contaminants falling onto the main optics. The telescope is then electronically connected and calibrates its GPS coordinates.

Before beginning observation, the telescope's pointing accuracy and focus are recalibrated. The telescope is slewed to a bright star (Betelgeuse was commonly used) and a low exposure image is taken. Based on the actual location of the star in the image and the assumed central location, the telescope is jogged to center the star in the FOV and synced to the correct RA and DEC. Refer to $\S 3.1 .4$ for information on the focusing process.

After telescope calibration, all current and viable TLEs are uploaded into TheSkyX software. Each observable pass was projected onto the viewing screen overlapped with the astronomical bodies as well as the relative location of the observatory, as seen in Fig.3.9. This created the desired RA and DEC angles of the object during its full observable transit while also simulating the apparent transit times at points along the arc. Using the desired epochs determined in the preparation stage using the STK software, both the necessary pointing angles of the telescope and desirable observation times within the orbit were defined. 


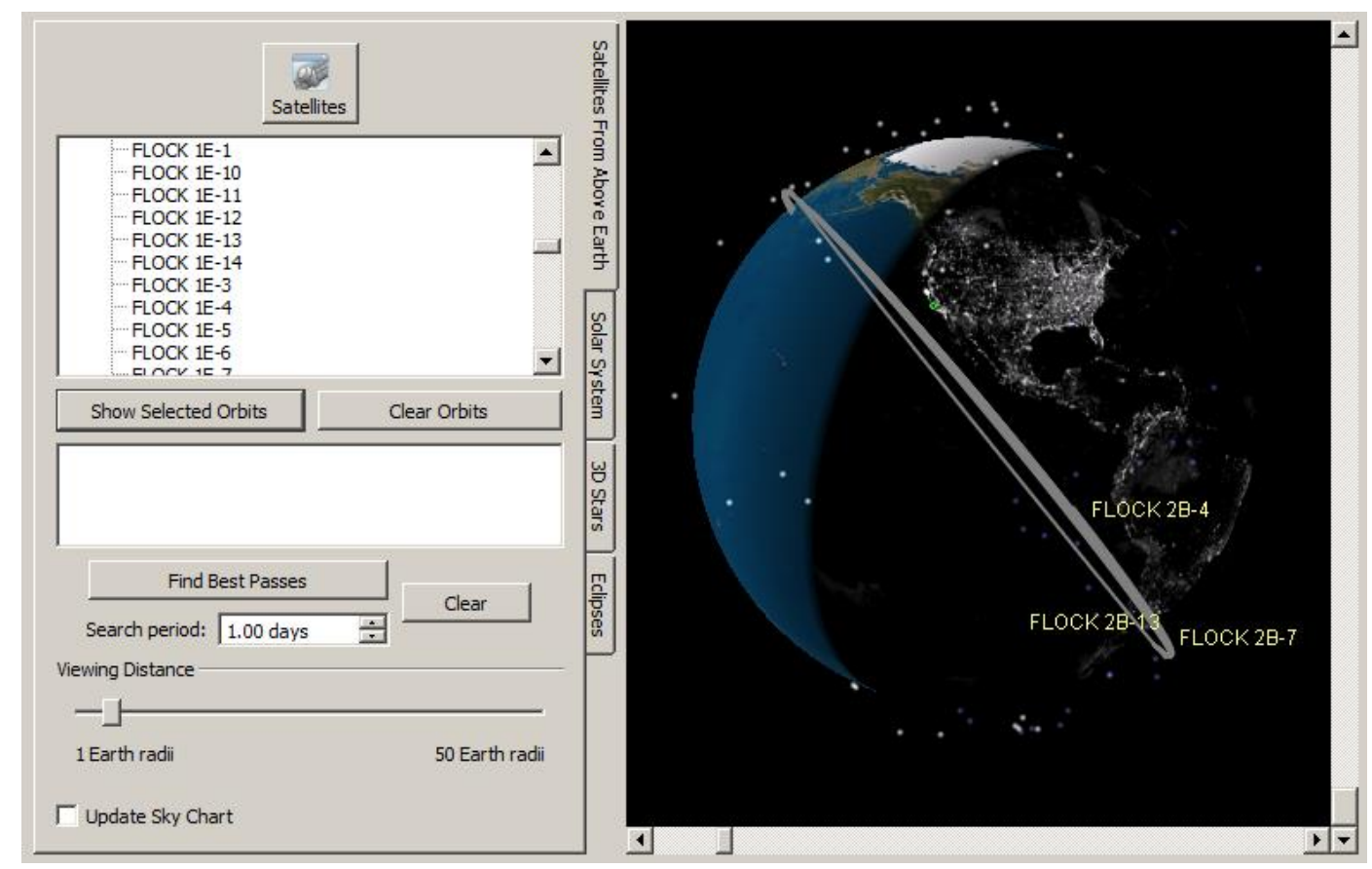

Figure 3.10: TheSkyX Transit Path

Once the telescope was focused on the correct RA and DEC location, a test image was first taken to calibrate the pointing accuracy using the local stars within the FOV. Once re-synced to minimize pointing error and the precise time of transit arrived, the image was taken.

All images implemented a 0.2 second exposure and $2 \mathrm{X} 2$ binning scheme. With a roughly 22 'x 15 ' arcminute FOV, the exposure time of 0.2 seconds allowed for the passing satellite to transit a fair distance while still having both starting and ending locations bounded within the FOV. This allowed for two separate data points to be utilized from a single image while also indicating a clear direction of travel. All images taken each night were saved in .FIT format and transferred to a file labeled to the particular date of observation. 
The 2X2 pixel binning allowed for a significantly reduced full frame download time. Since the visible transit paths were limited (usually just a few minutes), the time it took to download each frame was taking place within the critical observation time. By cutting the time for each frame download from $\sim 9$ seconds to $\sim 4$ seconds, more images were able to be taken in the small window of opportunity. Although the binning scheme decreased the resolution of the images, the error associated with the blurring effect caused by the satellite's motion wasn't significant enough to show a pronounced effect on the assumed central pixel as seen in Fig. 3.11. Additionally, the $2 \mathrm{X} 2$ binning also increased the visibility of the satellite on the images by averaging out the collected photons over a larger surface. This allowed for dimmer objects to be seen on the images more easily than the former $1 \mathrm{X} 1$ binning.

The outer diameter of the satellite trail stayed relatively constant throughout all observations, but with the $2 \mathrm{X} 2$ pixel binning, the standard deviation is enlarged and will have an increased effect on the associated errors. Although the error is marginally larger, the effects of these increases are relatively insignificant and can be justified in exchange for the benefits posed for the observation process used. A more in-depth explanation of the pixel and plate scale deviations and the error analysis process, please refer to Appendix E. 


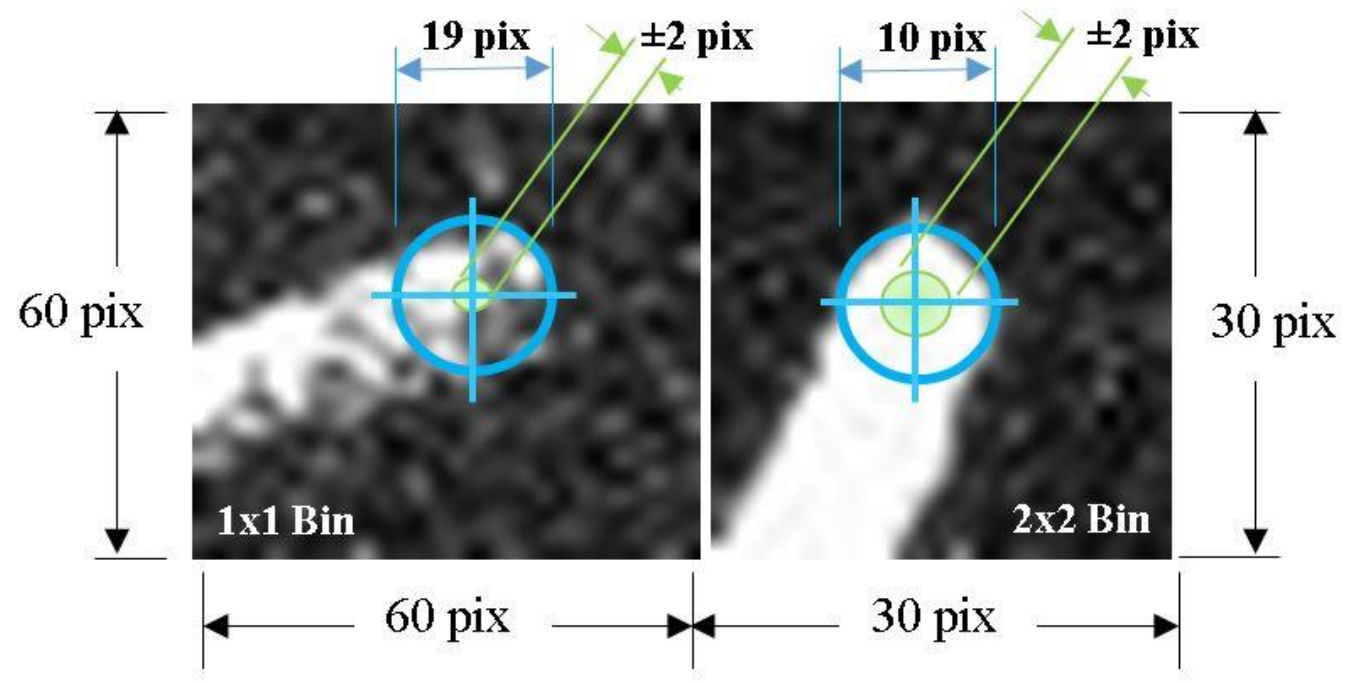

Figure 3.11: Binning Scheme

\subsubsection{Photometric Reduction}

The photometric reduction of an image is a process designed to negate as much of the inherent noise as possible within the image. The noise that is generated creates a less definable data set. The desired signal of the collected images are the unaltered photon count of each observed object in the FOV without distortion from outside effects. With a high signal-to-noise ratio (SNR), the desired signal is more clearly defined as depicted in Eq. $3.1[16]$.

$$
S N R_{d B}=10 \log _{10}\left(\frac{P_{\text {signal }}}{P_{\text {noise }}}\right)
$$

The three most accountable causes of noise that are generated within an image are charge accumulation noise, noise generated from dust or granular particulates on the main optics, and the background noise generated from the electronics within the system. The generated noise can be isolated in their own image and later subtracted from the scientific images taken each night. As seen in Fig. 3.11, the three image types are defined as: Dark, 
Bias, and Flat field images. Dark images are taken with a closed shutter and same exposure time as the scientific images in order to represent a noise count for the accumulated charge over the time of exposure. The Bias images are taken with a zero exposure time in order to account for any inherent noise being generated within the equipment itself. And lastly, the Flat field images are taken with an open shutter and same exposure as the scientific images while pointed at the sky just after sunset. This allows the observer to generate a monochromatic background in the FOV so that irregularities caused by any particles on the optics can be isolated.

A series of each image type is taken, combined and averaged in order to make a normalized "master image". The "Master Dark" and "Master Bias" images are then subtracted from the scientific images from each night and then divided by the "Master Flat Field" image. This process refines the images by minimizing the background noise.

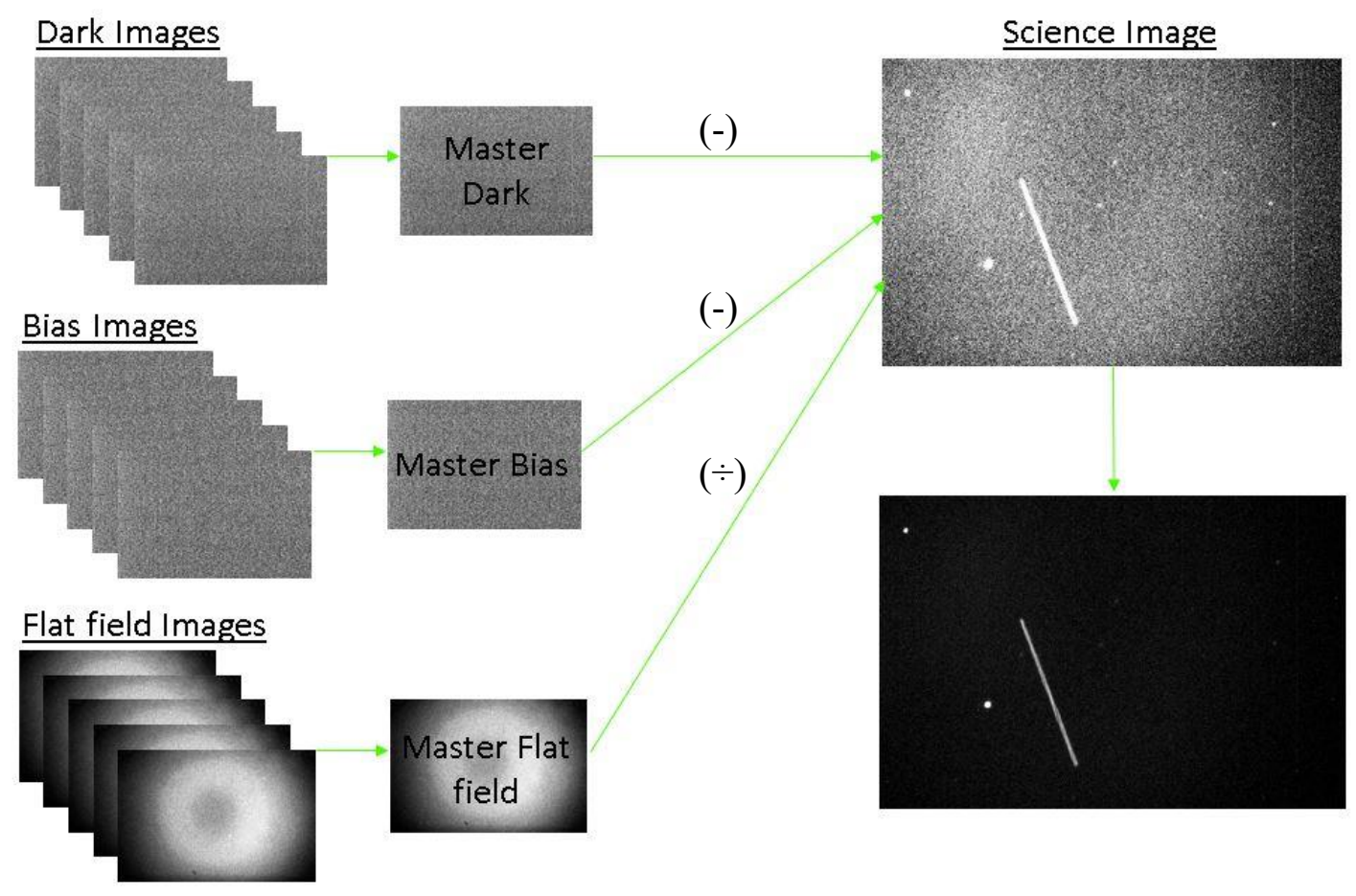

Figure 3.12: Photometric Reduction Process 
Although this process is useful in magnitude sensitive scenarios, it did not prove to be necessary for the orbital analysis of the larger Globalstar satellites. In the case of the observation of nanosatellites, this process would prove to be a useful, and perhaps necessary tool in helping to define the path of the passing object. Even after photometric reduction of the attempted images taken of the nanosatellites, there was no discernable distinction from the background which either indicated too faint of a light signature or likely a pointing and/or timing error. While the nanosatellites are faint, the relatively low orbit in comparison to the Globalstar satellites require them to travel much faster in orbit and are more difficult to observe. The nanosatellite imaging data and constraints will be discussed later in Chapter 5. 


\section{ANALYSIS}

\subsection{Image Review}

Over the span of a single night of observation, nearly 100 images were taken of roughly 15-20 satellites. These images were reviewed in the ds9 software designed specifically for .FIT image formatting. All images were viewed in a linear z-scale in order to increase the contrast between target objects from the background noise. All images containing either one or two end points of the passing satellites were saved to a separate file while all others were discarded.

It is common practice to use a comparison star as a datum point in space as a reference of a well-defined position to base all other points off of. Instead of the comparison star being determined prior to the observation of each pointing location, the continuous transit path was uploaded into TheSkyX software depicting the RA and DEC for the entire access time and used as the approximate location of the pass. With this information in tandem with illumination times provided by STK and calculation of the positional range with optimal SPA, an area along the transit path with a nearby star having an apparent 
magnitude below 8.0 was chosen and the image could be captured at the specific epoch in its transit across the FOV.

Although the file header provides the telescope's pointing RA and DEC of the center point of the image, it could not be used to accurately determine the relative location of the satellite due to the inherent pointing error within the mechanics of the telescope. However, the approximate pointing angles were input into the Aladin Sky Atlas so that the more accurate International Celestial Reference System (ICRS) RA and DEC values of the comparison star could instead be used to compare against the .FIT image. As seen in Fig 4.1, the left side illustrates the image of a passing satellite while the right side is the same RA and DEC shown in Aladin.

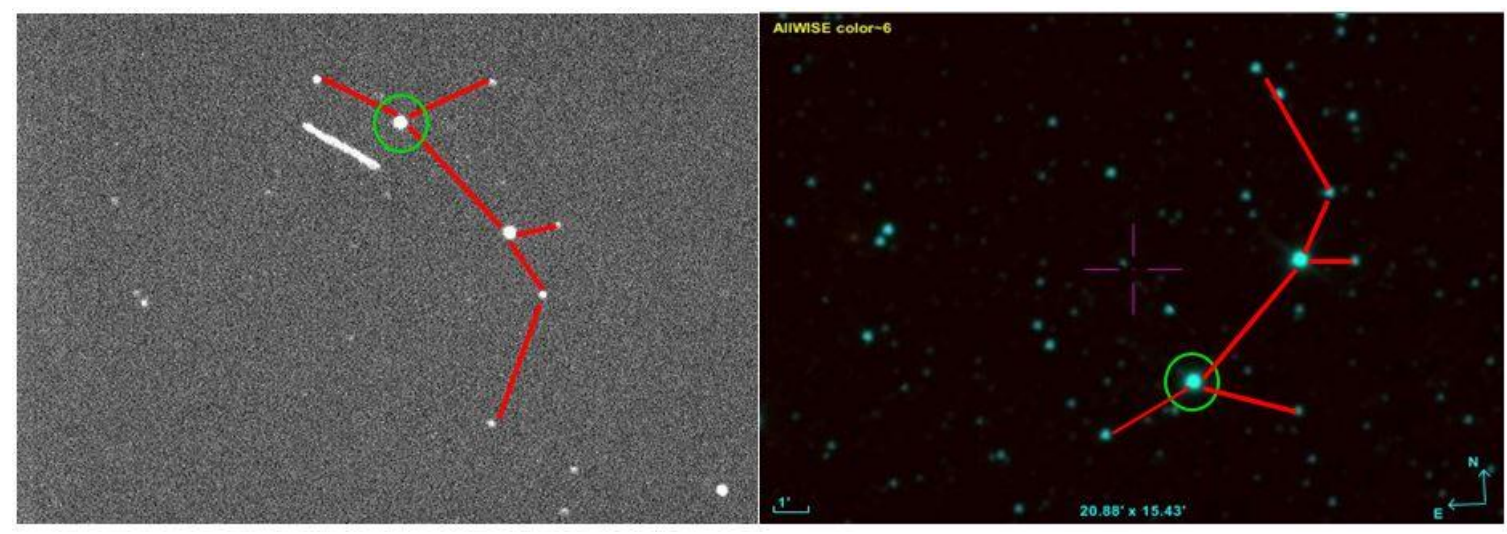

Figure 4.1: Star Constellation Pattern Recognition

The pattern recognition of the star constellations within the FOV were used to verify the comparison star identity of each image. The RA and DEC of the chosen comparison star was then recorded in an excel sheet and will be used later in determining the relative position of the satellite to the more accurate star coordinates. 


\subsection{Astrometry}

\subsubsection{Data Reduction}

The decomposition of the images into data sets began with identifying the relevant positions within the images. The ds9 software was used to open the .FIT image files and determine the $[\mathrm{X}, \mathrm{Y}]$ pixel coordinates of the chosen comparison star and the two end points of the satellite. Figure 4.2 depicts the ds9 Graphic User Interface and the locating of the necessary pixel values.

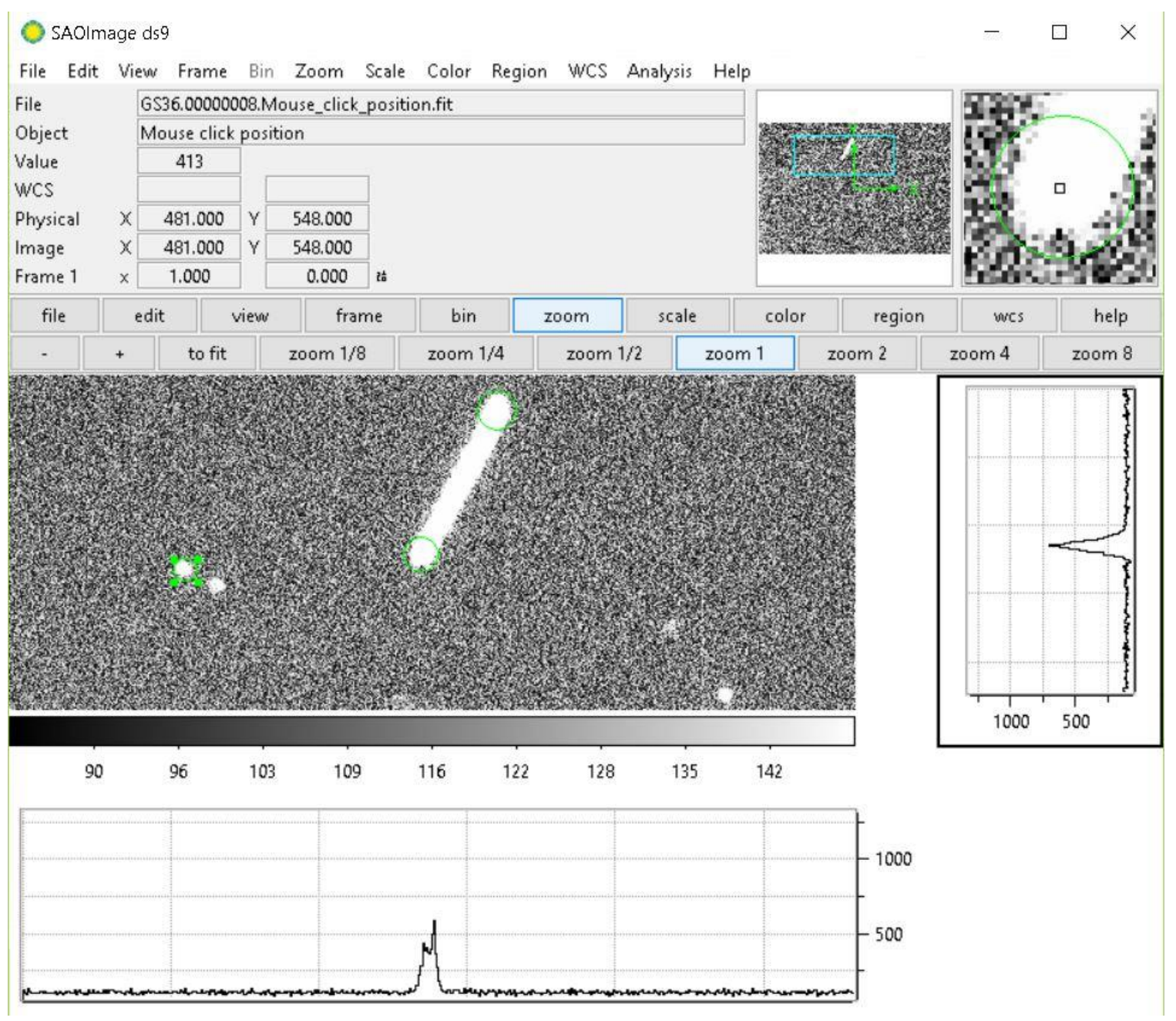

Figure 4.2: ds9 Image Viewer and Pixel Location 
Both the horizontal and vertical photon graphs are used to pin point the max photon count across both axis in order to accurately determine the center point of the satellite. The cursor is moved across the highlighted areas of interest on the image in order to determine the max peak on both axis closest to the satellite end points. The maximum photon count along the orbital path should indicate the central location of the satellite and minimize the averaged scintillation effects of the atmosphere.

Since there is a finite allowable resolution of the CCD camera pixels and unpredictable atmospheric effect, an uncertainty of \pm 1 pixel was given to the star position, and \pm 2 pixels for the satellite positions. The larger uncertainty for the satellite positions are due to a pronounced blurring effect caused by the satellite's motion, which was also prevalent in Brock Schmalzel's data [19].

Due to the fact that multiple images were captured along the orbital plane for all satellites, the time stamp on each images indicated the general direction of the satellite as it passed through the FOV. The RA and DEC values associated with each of these images allowed for the determination of the direction motion and was verified with initial STK propagations. In order to obtain the required data for further analysis, the pixel locations of the satellites must also be converted to RA and DEC angle sets. This is accomplished by using the companion star as the point of reference and the previously mentioned direction of motion of the satellite as the reference orientation.

The STK simulated orbital paths of the satellites mentioned in $\$ 3.2 .2$ are used as the predicted slope direction of motion defined by Eq.4.1. 


$$
m_{P}=\frac{\delta_{N}-\delta_{T}}{\alpha_{N}-\alpha_{T}}
$$

The subscripts $T$ denotes the RA/DEC of the target location at the precise time of the actual captured image while the $N$ subscript denotes the next target location with the additional 0.2 exposure time later. Replacing the predicted RA/DEC positions in Eq.4.1 with the $[\mathrm{X}, \mathrm{Y}]$ pixel values for the two satellite positions, the actual FOV slope was found through Eq. 4.2.

$$
m_{A}=\frac{\operatorname{pix}_{Y_{2}}-p i x_{Y_{1}}}{\operatorname{pix}_{X_{2}}-p i x_{X_{1}}}
$$

To transfer from the pixel frame to the RA/DEC frame, the following rotation shown in Fig.4.3 was made through the correction angle, $\theta$. Any error in the predicted slope due to an imperfect TLE propagation was assumed to be negligible at this scale.

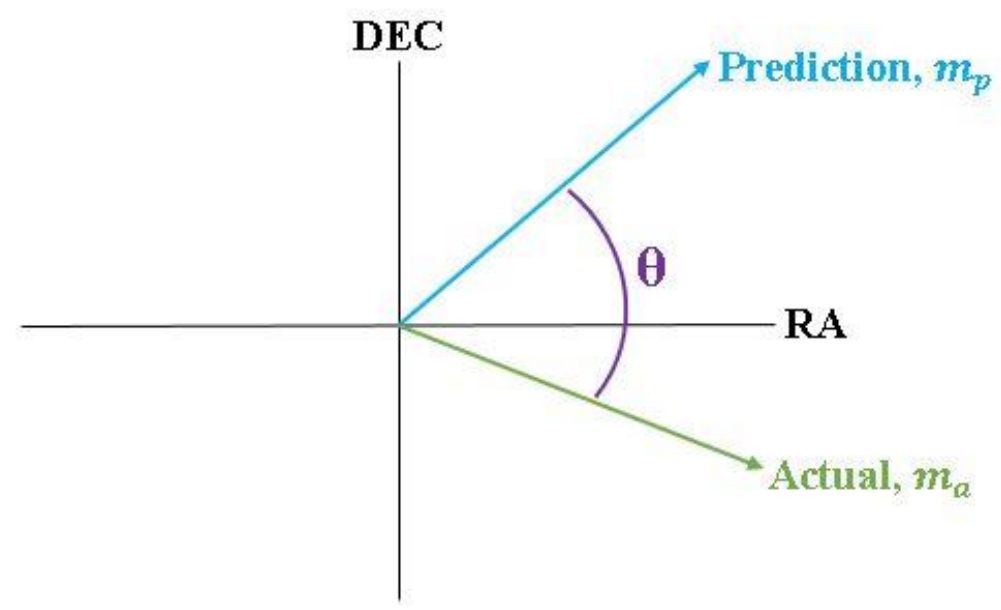

Figure 4.3: Frame Rotation

The necessary rotation angle can be found through Eq.4.3 seen below. Once the pixel frame has been rotated to the correct RA/DEC orientation, the corresponding angle values can be determined using Eq.4.4. 


$$
\begin{gathered}
\theta=\tan ^{-1}\left(m_{A}\right)-\tan ^{-1}\left(\frac{m_{P}}{\cos \left(\delta_{\text {mean }}\right)}\right) \\
{\left[\begin{array}{l}
\delta \\
\alpha
\end{array}\right]_{\text {sat }}=\left[\begin{array}{l}
\delta \\
\alpha
\end{array}\right]_{\text {star }}+\left[\begin{array}{cc}
\cos (\theta) & -\sin (\theta) \\
\sin (\theta) & \cos (\theta)
\end{array}\right]\left[\begin{array}{c}
\Delta_{Y} \\
\frac{\Delta_{X}}{\cos \left(\delta_{\text {mean }}\right)}
\end{array}\right] P S}
\end{gathered}
$$

The $\Delta_{X}$ and $\Delta_{Y}$ values are the spatial differences between the comparison star and each point of the passing satellite and is visually represented in Fig.4.4 below. The $\cos \left(\delta_{\text {mean }}\right)$ term in Eq.4.3 and 4.4 were used to scale the RA values for the change in DEC.

Referring back to Fig.2.3, you can see that as declination increases away from the celestial equator, an angular distance corresponding to the change in right ascension decreases, due to its spherical shape. Therefore, the change in RA must be scaled by the mean DEC angle at which the observation occurs. The plate scale value, $P S$, is the scaling factor used to convert from pixel quantity to the associated arcminutes distance representation. Since the image FOV is roughly $22^{\prime} \mathrm{X} 15^{\prime}$ arcminutes and the CCD pixel resolution with a $2 \mathrm{X} 2$ binning is $1092 \mathrm{X} 736$, the PS turned out to be 1.21 arcseconds/pixel. The process of determining the satellite's RA/DEC angles from the recorded images were calculated using MATLAB [23].

It is important to note that in the case of imaging a satellite without the use of a reference TLE, the predicted slope is not known. Instead of referencing the frame rotation off of the predicted satellite slope, the relative pixel locations of a constellation of stars within the image can be corresponded to their RA and DEC values found in an astronomical database. The difference between the pixel locations and the RA and DEC values can alternatively be used to correct the reference frame. 


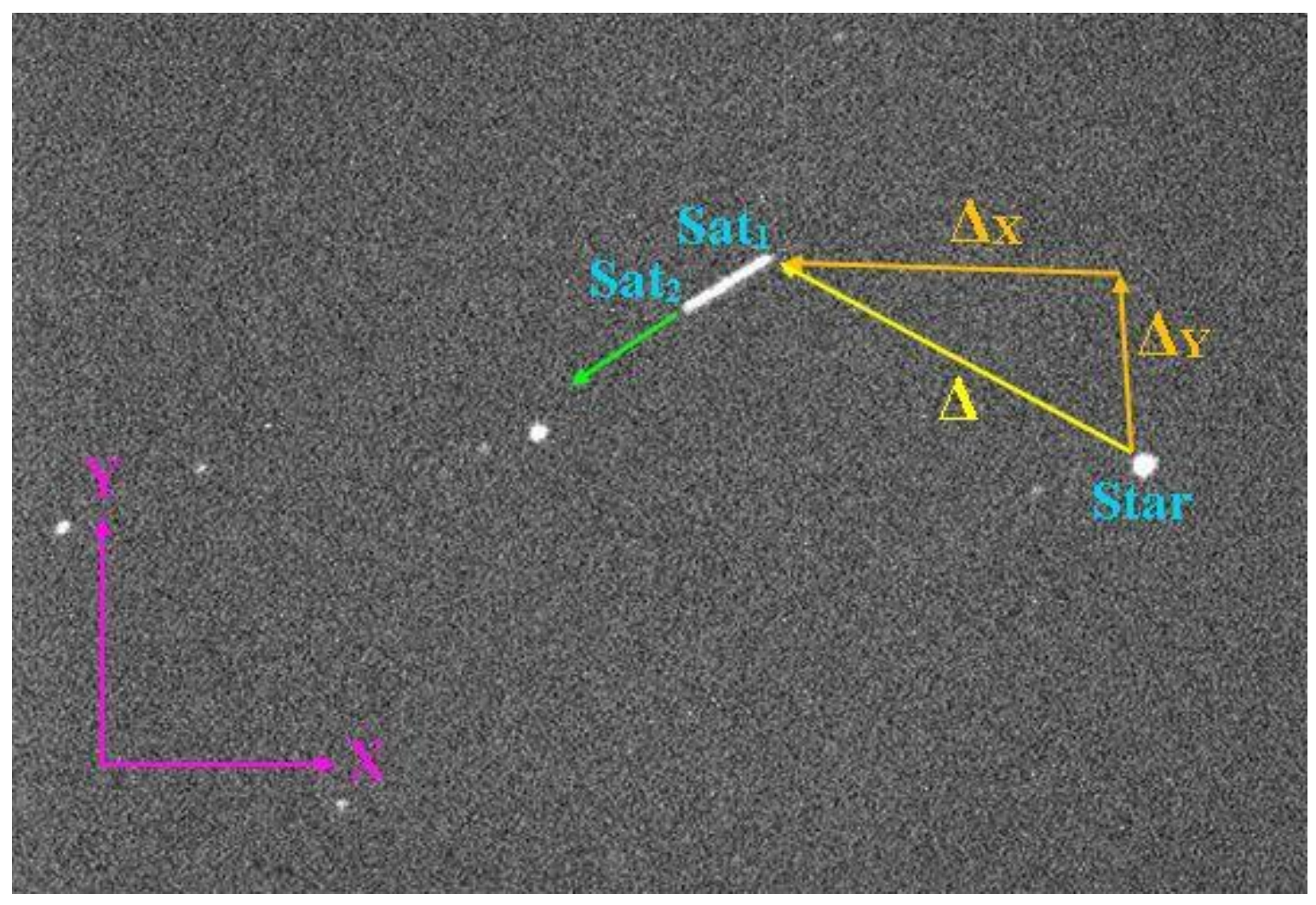

Figure 4.4: Satellite Motion and Pixel Decomposition

\subsubsection{Orbit Determination}

All orbital determination techniques used in this thesis require at least three observation points for preliminary calculations. These three points are analyzed using the two angles-only methods previously stated in $\$ 2.2$ which are available online in $\mathrm{C}++$, Fortran, Pascal, and MATLAB languages [19].

These functions require the ground site vectors designating the point of observation, the times the images were taken of each of the three data point locations, and the line of site vectors, $\widehat{L}$, seen in Eq. 2.1, which were calculated from the previously determined RA and DEC of each data point. 
The timestamp attached to each images header file was converted to its corresponding Julian Date and used for all Sat ${ }_{1}$ locations. For all Sat 2 data points of each image, the 0.2 second exposure time was added to the timestamp and similarly converted to the corresponding Julian Date. The ground site vectors of the Cal Poly Observatory location were found using Vallado's MATLAB code to locate the Earth-Centered EarthFixed (ECEF) position vectors and converted into the Earth-Centered Inertial (ECI) frame [32]. I then implement the IOD and OD operations to output the position and velocity vectors of the input data and converted into COEs for comparison with the original TLE COE values.

For the scenarios that allowed six or more images to be taken of a single satellite, more advanced methods could be used to more accurately determine their orbits. These two methods include an Extended Kalman Filter and Non-Linear Least Squared methods.

Refer to $\S 2.3$ for a deeper explanation of the EKF and LS processes. The implementation of these OD methods were processed using the MATLAB [19].

\subsubsection{TLE Positional Error}

It is well known that as an orbit is propagated further into time, the error associated with the projected orbit becomes larger. The models attempting to replicate the effects of the various perturbation effects on an orbiting body but are imperfect and increase in error to the point of unreliability. These variations cause a divergence from the predicted location in which can be compared to the actual location taken via optical observation. To determine 
the distance between the predicted direction of movement and the actual observed path, $D$, Eq.4.6 and Eq.4.7 were used.

$$
\begin{gathered}
b_{A}=\delta_{1}-m_{A} \alpha_{1} \\
D=\frac{\left|\delta_{T}-m_{A} \alpha_{T}-b_{A}\right|}{\sqrt{m_{A}^{2}+1}}
\end{gathered}
$$

Using the slope determined in Eq.4.2, we can find the "y-intercept" for the direction of motion, $b_{A}$. This distance is then converted into the corresponding angular CTE seen in Eq.4.8, where $\mathrm{R}$ is the range of each observed object.

$$
C T E=\tan (D) R
$$

In order to determine the ATE between the actual observed time and the projected time, the recorded timestamp and the projected pass are required. The ATE was found using Eq.4.9.

$$
A T E=t_{E} \sqrt{\frac{\mu}{r_{E}+h}}
$$

The ATE is dependent on the predicted and actual velocity values of the orbiting object, the variation in the altitude of the satellite, $h$, has a direct correlation to the satellite's velocity. The $t_{E}$ variable is the time error between the predicted time at which the satellite crosses the FOV and actual recorded timestamp of the image taken. 


\subsubsection{Magnitude Feasibility}

The magnitude of the object being observed and the limiting magnitude of the telescope are two values that must overlap in order to be able to visibly image a passing satellite. The predicted apparent magnitudes of the nanosatellites being observed were determined through the use of STK and a developed MATLAB function taking in a range of major contributing variables that affect the visibility of a satellite. These variables include the satellite albedo (assumed to be 0.175), satellite dimensions, and atmospheric extinction. The attempt in viewing the FLOCK nanosatellite system arose from the increase in reflective surface area after solar panel deployment. The predicted apparent magnitudes of these satellites are $\sim 12.6$ for an un-deployed $3 \mathrm{U}$ CubeSat and $\sim 11.2$ with solar panels deployed. With the inclusion of some nanosatellites such as the CP5 CubeSat, deployable solar sails can increase the predicted apparent magnitude to 6.99 [23].

It is additionally necessary to know the instrumental magnitude limit of the telescope used in the observation process. Seen in Fig 4.5, Ackerman et al. estimated the limiting magnitudes of variable telescope sizes for a stationary telescope pointing position with the satellite passing through the FOV where the green line indicates the Cal Poly telescope aperture diameter of $355.6 \mathrm{~mm}$ [35]. The limiting visual magnitudes obviously increase in magnitude as the aperture diameter of the telescope increases due to the increased amount of photons being collected. The variation in the four orbital altitudes are less intuitive in the sense that as the orbital altitude increases, the limiting magnitude becomes larger. Since the satellites at a higher altitude are transiting the FOV at a slower rate, the collected photons are spread over a fewer amount of pixels creating a larger variation from the background noise. 


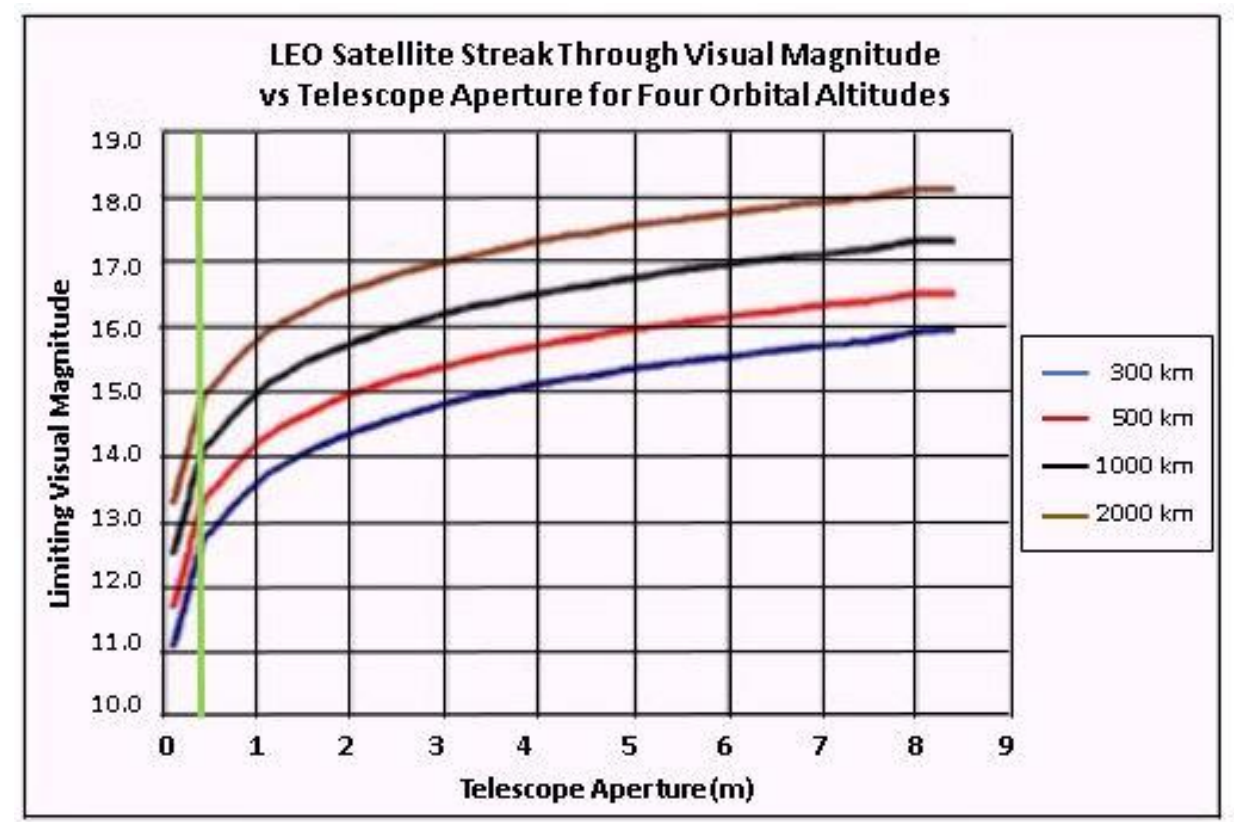

Figure 4.5: Limiting Magnitudes of a Staring System [23]

Although the decrease in the altitude of the satellite has a positive effect on the perceived brightness, the speed associated with the lower orbits have an adverse effect on the visibility of the passing objects. Using a 0.2 second exposure for all images, the collected photons can be considered relatively constant. With the velocity increasing as the altitude lowers, the constant photon count spreads across more pixels, as portrayed in Fig. 4.6.

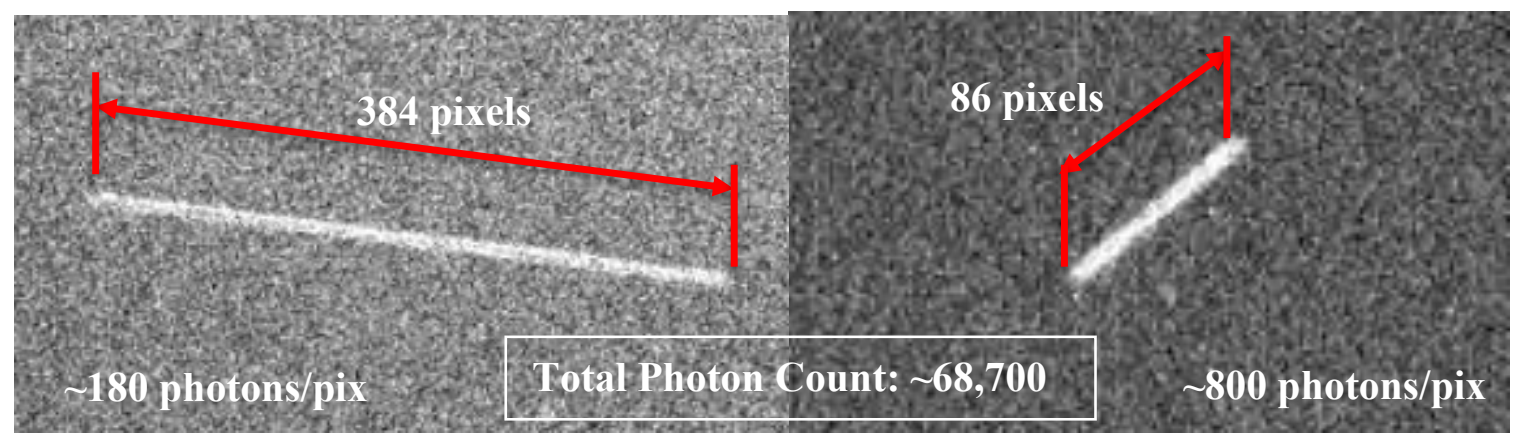

Figure 4.6: Different Altitude Satellite Image Photons per Pixel 
The lower amount of photons per pixel diminish the definable borders for a passing object and even the potential to render an object unobservable. Given constant reflective surface area of the satellite, the limiting visual magnitude seen in Fig.4.5 increases as altitude increases due to this fact.

Since all of above predicted apparent magnitudes are at an altitude $800 \mathrm{~km}$ and above, they lie within the theoretical telescope limiting magnitude but will additionally require optimal conditions to increase the probability of a successful image capture. With the assumption that the error in the cross track direction is not significant enough for the object to be outside the FOV, the probability of a successful image capture is still feasible. 


\section{RESULTS AND DISCUSSION}

Observations began March $16^{\text {th }} 2016$ and lasted until April $6^{\text {th }}$ 2016. A series of 20 satellites were observed and roughly 100 data points were taken within this time frame. The entirety of the observed satellites were taken from the Globalstar satellite system. There were observation attempts made on multiple nanosatellites, but due to orbital position and observation timing with consideration of undesirable weather factors and scheduling conflicts (observatory was shared between multiple research projects), none were successful. Depicted in Table 5.1 are just one of the sample sets of nanosatellites for a given night of observation attempts made on the most desirably visible nanosatellites at that time. Although the sunset was estimated to occur at 3:37:00 UTC on the night of May $7^{\text {th }} 2016$, the astronomical twilight didn't occur until roughly 3:55:00-4:05:00 UTC. Most of the "observable transits" occurred during the last minutes of daylight which effectively rendered the majority of the targets unobservable. The remaining target nanosatellite transit times that occurred after or very near the full setting of the Sun were below or bordering the minimum elevation of $25^{\circ}$ due to the surrounding buildings. The values in Table 5.1 are shaded red and green to indicate the satellite's orbit requirements being met for acceptable transit times and elevation angles. Some of the data points may seem to be 
theoretically observable, but with such a minimal access period available for viewing, it was not possible to acquire a reliable image.

Table 5.1: Nanosatellite Observation Sample Set

\begin{tabular}{|c|c|c|c|}
\hline Sat ID & Sun Set (UTC) & Access Period (UTC) & Elevation $\left(^{\circ}\right.$ ) \\
\hline FLOCK 2B-2 & $3: 52: 46$ & $3: 50: 01-3: 52: 55$ & 25.3 \\
\hline FLOCK 2B-3 & $3: 57: 50$ & $3: 55: 21-3: 58: 09$ & 23.4 \\
\hline FLOCK 2B-5 & $4: 02: 38$ & $4: 00: 38-4: 02: 43$ & 21.1 \\
\hline FLOCK 2B-6 & $3: 40: 15$ & $3: 36: 50-3: 40: 19$ & 35.1 \\
\hline FLOCK 2B-9 & $3: 51: 02$ & $3: 48: 06-3: 51: 16$ & 29.8 \\
\hline FLOCK 2B-14 & $3: 37: 44$ & $3: 34: 08-3: 37: 33$ & 28.3 \\
\hline PSAT_40654 & $3: 36: 28$ & $3: 28: 01-3: 32: 02$ & 12.2 \\
\hline ULTRASat5 & $3: 24: 54$ & $3: 16: 38-3: 21: 34$ & 24.8 \\
\hline ULTRASat10 & $3: 57: 33$ & $3: 50: 27-3: 54: 26$ & 9.9 \\
\hline
\end{tabular}

Although the minimal altitude of the objects positioned in a LEO orbit allow for an increased apparent magnitude, it significantly decreases the access time and illumination period available for observation. Even though no nanosatellite images were gained within the period of observation, this was only due to the unfortunate misalignment of the RAAN of the orbiting bodies relative to the point of observation given the allowable times of observation. Even though the orbital period of the targets are roughly 90 minutes and could pass overhead multiple times per night, each subsequent orbit of those listed above shift westward and in turn lowers the elevation even further below the acceptable limit. The position of the nanosatellites for each subsequent day similarly started at a lower elevation than the previous. Additionally, due to the extremely faint apparent magnitudes, the light of a full moon on a night of observation can potentially drown out the light signature from a passing nanosatellite and must be avoided when possible. 
Even with all of these obstacles to overcome, there is still a high feasibility of a successful nanosatellite observation and orbit determination using the Cal Poly optical telescope. With the instrumentation available, the minimum observable apparent magnitude is still within the limitations of the telescopes capabilities [3]. Based on the specifications and theoretical capabilities of the instrumentation used, only the correct conditions for observation are needed in order to image such faint objects.

The orbit determination techniques applied to the independently collected data on the Globalstar system depicted in the next sections verify the relative accuracy that can be achieved without the aid of external sources as long as an adequate initial observation is provided or produced. Options for initial observation techniques are explored briefly in the "future work" section.

\subsection{Orbital Determination}

In presenting the results of the data, direct comparisons between the COEs as well as state vectors will be made between the reference TLE and the solutions acquired from the IOD methods. The reference TLEs were converted from the mean orbital elements to osculating through the use of Kwok's method [32]. Table 5.2 shows the comparison between the COE values of three single trio-captures. The remaining values can be found in Table C.1 in Appendix C. The difference in the initial TLE epochs and the observation epochs seen in Table 5.2 are to indicate when the initial TLE was last created and the time at which the observations were made. 
The initial TLEs were propagated forward to the time of the observation using SGP4 perturbation models in order to ensure the most accurate prediction of their location.

Table 5.2: Comparison of COEs through angles-only IOD to reference TLE

\begin{tabular}{|c|c|c|c|c|c|c|c|}
\hline Satellite ID & Source & Epoch (UTC) & $a(\mathbf{k m})$ & $\mathbf{e}$ & $i\left({ }^{\circ}\right)$ & $\Omega\left({ }^{\circ}\right)$ & $\omega\left(^{\circ}\right)$ \\
\hline \multirow{4}{*}{ Globalstar M030 } & TLE & $3 / 15$ 19:24 & 8270.5 & 9.310E-04 & 51.917 & 296.001 & 102.9 \\
\hline & Vallado Gauss & \multirow{3}{*}{$3 / 163: 27$} & 9046.0 & $9.63 \mathrm{E}-02$ & 48.439 & 295.681 & 210.673 \\
\hline & Curtis Gauss & & 9044.4 & $9.63 \mathrm{E}-02$ & 48.439 & 295.685 & 210.736 \\
\hline & Double-r & & $\begin{array}{r}9044.4 \\
\pm 22.005 \\
\end{array}$ & $\begin{array}{c}9.63 \mathrm{E}-02 \\
\pm 1.4 \mathrm{E}-3 \\
\end{array}$ & $\begin{array}{l}48.439 \\
\pm 0.004 \\
\end{array}$ & $\begin{array}{c}295.685 \\
\pm 9.314 \\
\end{array}$ & $\begin{array}{l}210.739 \\
\pm 0.0983 \\
\end{array}$ \\
\hline \multirow{4}{*}{ Globalstar M037 } & TLE & 4/5 3:26 & 7788.7 & $6.37 \mathrm{E}-04$ & 51.956 & 334.714 & 51.590 \\
\hline & Vallado Gauss & \multirow{3}{*}{ 4/6 4:19 } & 7760.7 & $7.35 \mathrm{E}-03$ & 51.392 & 332.275 & 262.706 \\
\hline & Curtis Gauss & & 7753.2 & $6.29 \mathrm{E}-03$ & 51.449 & 332.322 & 264.206 \\
\hline & Double-r & & $\begin{array}{l}7753.2 \\
\pm 0.808 \\
\end{array}$ & $\begin{array}{r}6.29 \mathrm{E}-03 \\
\pm 3.953 \mathrm{E}-5 \\
\end{array}$ & $\begin{array}{c}51.449 \\
\pm 1.79 \mathrm{E}-4 \\
\end{array}$ & $\begin{array}{r}332.322 \\
\pm 10.522 \\
\end{array}$ & $\begin{array}{r}264.214 \\
\pm .8331 \\
\end{array}$ \\
\hline \multirow{4}{*}{ Globalstar M053 } & TLE & 4/1 14:50 & 8401.0 & $7.88 \mathrm{E}-04$ & 52.070 & 124.403 & 100.680 \\
\hline & Vallado Gauss & \multirow{3}{*}{$4 / 24: 35$} & 7793.1 & $7.16 \mathrm{E}-02$ & 52.159 & 122.877 & 220.288 \\
\hline & Curtis Gauss & & 7769.0 & $7.25 \mathrm{E}-02$ & 52.222 & 122.838 & 219.566 \\
\hline & Double-r & & $\begin{array}{r}7769.0 \\
\pm 6.5011 \\
\end{array}$ & $\begin{array}{c}7.25 \mathrm{E}-02 \\
\pm 4.511 \mathrm{E}-4\end{array}$ & $\begin{array}{c}52.222 \\
+5.842 \mathrm{E}-4\end{array}$ & $\begin{array}{c}122.838 \\
\pm 6.545 \\
\end{array}$ & $\begin{array}{l}219.566 \\
\pm 0.0641\end{array}$ \\
\hline
\end{tabular}

The semi-major axis data in the table above is an indication that the variation in orbit altitude and shape differ the most drastically in comparison to the initial TLE values. The large difference in the eccentricity calculated in Globalstar M030 and M053 is the most likely contributor to the large change in semi-major axis values. It became apparent, as you will see in Table 5.4, that the addition of multiple trio-capture data sets dramatically decrease the eccentricity values. With the hardware that was used, a single trio-capture is simply not reliable enough to ensure a consistently accurate estimation of a satellite's orbit.

The Globalstar M037 data is much more accurate in large part due to the fact that this observation arc passed at an ideal $\sim 45^{\circ}$ elevation angle to the telescopes pointing position. It seemed that the further the telescope was required to rotate itself from its standard orientation, the larger the corrective reference frame rotation was needed. Since the frame rotation utilized the slopes of the satellites as reference points, the error 
associated with the frame rotation would compound as the required rotation angle grew. It is now realized that an initial frame rotation using three well defined stars from the Aladin software and correlated with three stars within the image frame, the rotation error would be minimized. The uncertainties associated with the satellite end points were larger than that of the well-defined star locations. For future projects, I highly suggest the use of multiple reference stars.

The uncertainty values associated with the Double-r method are derived from the pixel selection process. A Monte-Carlo simulation was used in order to analyze the associated error which is further explained in Appendix E. Using this method, minute variances were added to both the time stamps and pixel locations of each observed data point over 10,000 iterations in order to determine the effects of pixel selection and plate scale error on the single-trio angles-only observations. Uncertainty values were only given to the Double-r method, as the difference in results of the various orbit determination methods (difference in both COE's and COE uncertainties) were found to be insignificant. For the same reason, only the Double-r method was used for the testing of the EKF and LS methods [23].

Due to this range in possible trio-capture arc lengths, the variation of the resulting estimated eccentricities of the orbits can cause large deviations in the other COEs. Table 5.2 shows that this deviation causes particularly high changes in the argument of perigee $(\omega)$. This is due to the perigee of the orbit being increasingly ill-defined as the eccentricity approaches zero.

The reason for the seemingly consistent variation of all three IOD methods in relation to the initial TLE is due to the inclusion of the SGP4 perturbation models in the 
initial TLE propagations previously stated. These perturbation models were not present in the OD or IOD methods used. However, with the initial TLE estimates propagated to the approximate observation times, the presumed perturbation effects were minimized.

Table 5.3 shows the comparison of the position and velocity vectors between the reference TLE and the Double-r method values. These state vector calculations of the same data sets presented in Table 5.2 were included as an attempt to determine potential sources of error responsible for the deviations encountered in the $\mathrm{COE}$ calculations. The error percentage along each directional coordinate and the coefficient of determination $\left(\mathrm{R}^{2}\right)$ between the Double-r method uncertainties and the error between the original TLE and the IOD values are included. The correlation between these two values were meant to determine a link between the $3 \sigma$ uncertainty errors determined through the use of the Monte-Carlo method and the error difference between the initial and observed data.

Table 5.3: Comparison of Propagated State Vectors

\begin{tabular}{|c|c|c|c|c|c|c|c|}
\hline Satellite ID & Source & $\mathbf{r}_{\mathbf{x}}(\mathbf{k m})$ & $\mathbf{r}_{\mathbf{y}}(\mathbf{k m})$ & $\mathbf{r}_{\mathrm{z}}(\mathbf{k m})$ & $\mathbf{v}_{\mathbf{x}}(\mathbf{k m} / \mathbf{s})$ & $\mathrm{v}_{\mathbf{y}}(\mathrm{km} / \mathrm{s})$ & $\mathbf{v}_{\mathrm{z}}(\mathrm{km} / \mathrm{s})$ \\
\hline \multirow{4}{*}{ Globalstar M030 } & TLE & -5193.61 & 5866.12 & -2653.36 & -2.2607 & -4.2532 & -4.9977 \\
\hline & Double-r & $\begin{array}{c}-5115.71 \\
\pm 0.196\end{array}$ & $\begin{array}{c}5734.68 \\
\pm 1.686 \\
\end{array}$ & $\begin{array}{c}-2885.22 \\
\pm .848\end{array}$ & $\begin{array}{l}-2.2249 \\
\pm .2 .6 \mathrm{E}-4\end{array}$ & $\begin{array}{c}-4.3867 \\
\pm 2.949 \mathrm{E}-4 \\
\end{array}$ & $\begin{array}{l}-4.8947 \\
\pm 2.9 \mathrm{E}-3 \\
\end{array}$ \\
\hline & Error (\%) & 1.50 & 2.24 & 8.74 & 1.58 & 3.14 & 2.06 \\
\hline & $\mathbf{R}^{2}$ & \multicolumn{6}{|c|}{0.125} \\
\hline \multirow{4}{*}{ Globalstar M037 } & TLE & 4851.08 & -5131.31 & -3281.16 & 5.0559 & 1.7304 & 4.7592 \\
\hline & Double-r & $\begin{array}{l}4587.52 \\
\pm 0.0254 \\
\end{array}$ & $\begin{array}{l}-5316.24 \\
\pm 0.0919 \\
\end{array}$ & $\begin{array}{l}-3235.28 \\
\pm 0.0249 \\
\end{array}$ & $\begin{array}{c}5.1892 \\
\pm 2.368 \mathrm{E}-4 \\
\end{array}$ & $\begin{array}{r}1.7385 \\
\pm 3.025 \mathrm{E}-5 \\
\end{array}$ & $\begin{array}{c}4.7337 \\
\pm 2.032 \mathrm{E}-5 \\
\end{array}$ \\
\hline & Error (\%) & 5.43 & 3.60 & 1.40 & 2.64 & 0.47 & 0.54 \\
\hline & $\mathbf{R}^{2}$ & \multicolumn{6}{|c|}{0.242} \\
\hline \multirow{4}{*}{ Globalstar M053 } & TLE & 3735.65 & -7396.02 & 1407.13 & 4.2403 & 1.1269 & -5.3063 \\
\hline & Double-r & $\begin{array}{l}4188.11 \\
\pm 0.4769 \\
\end{array}$ & $\begin{array}{l}-8379.16 \\
\pm 0.0716 \\
\end{array}$ & $\begin{array}{r}1625.54 \\
\pm 0.0966 \\
\end{array}$ & $\begin{array}{c}4.0044 \\
\pm 1.674 \mathrm{E}-4 \\
\end{array}$ & $\begin{array}{c}1.1920 \\
\pm 1.0 \mathrm{E}-4 \\
\end{array}$ & $\begin{array}{c}-4.8909 \\
\pm 9.149 \mathrm{E}-4 \\
\end{array}$ \\
\hline & Error (\%) & 10.8 & 11.7 & 13.4 & 5.9 & 5.5 & 8.5 \\
\hline & $\mathbf{R}^{2}$ & \multicolumn{6}{|c|}{0.185} \\
\hline
\end{tabular}

The low $\mathrm{R}^{2}$ values indicate a disassociation between the errors of each positional and velocity vector and the difference from the original TLE. This result could indicate a potential unseen cause of error that are affecting the state vector results. A notable trend 
present in the state vector data is the increased error in the positional vector values as opposed to the velocity vector values. This leads me to believe the source of the error may lie somewhere either within the data reduction software or the previously mentioned frame rotation error. The $\mathrm{R}^{2}$ values determined by Schmalzel using the same errors and uncertainty parameters ranged from a 0.17 to 0.40 correlation. Although he did detect a stronger correlation, a max of a $0.40 \mathrm{R}^{2}$ value is not a particularly strong or definitive causal relationship [23].

Once a solution was obtained from the IOD methods above, subsequent data sets from the same satellites were included at an attempt to refine the data with the two OD methods defined in $\$ 2.3$. The results from the EKF and LS methods are shown below in Table 5.4 in relation to the original TLE value and Double-r IOD method values. The epoch times of the EKF and LS methods presented in the table below differ from those of the Double-r epochs to indicate the timestamp of the final data sets used in the OD calculations.

Table 5.4: Comparison of EKF and LS OD Methods

\begin{tabular}{|c|c|c|c|c|c|c|c|}
\hline Satellite ID & Source & Epoch (UTC) & $a(\mathrm{~km})$ & $\mathbf{e}$ & $i\left(^{\circ}\right)$ & $\Omega\left({ }^{\circ}\right)$ & $\omega\left(^{\circ}\right)$ \\
\hline \multirow{4}{*}{$\begin{array}{l}\text { Globalstar } \\
\text { M053 }\end{array}$} & TLE & 4/1 14:50 & 8401.0 & $7.88 \mathrm{E}-04$ & 52.070 & 124.403 & 100.680 \\
\hline & Double-r & $4 / 24: 35$ & 7769.0 & $7.25 \mathrm{E}-02$ & 52.222 & 122.838 & 219.566 \\
\hline & EKF & \multirow{2}{*}{ 4/2 4:43 } & 8435.7 & $8.63 \mathrm{E}-03$ & 52.160 & 124.920 & 246.447 \\
\hline & LS & & 8413.02 & $8.29 \mathrm{E}-03$ & 52.192 & 125.103 & 165.246 \\
\hline \multirow{4}{*}{$\begin{array}{c}\text { Globalstar } \\
\text { M056 }\end{array}$} & TLE & $3 / 164: 12$ & 8039.3 & $9.11 \mathrm{E}-04$ & 52.087 & 78.944 & 84.489 \\
\hline & Double-r & $3 / 173: 28$ & 7076.5 & $6.72 \mathrm{E}-02$ & 51.345 & 74.505 & 218.114 \\
\hline & EKF & \multirow{2}{*}{$3 / 173: 33$} & 7934.4 & $7.90 \mathrm{E}-03$ & 52.355 & 79.679 & 359.838 \\
\hline & LS & & 7903.9 & $1.04 \mathrm{E}-03$ & 52.399 & 79.745 & 202.872 \\
\hline \multirow{4}{*}{$\begin{array}{c}\text { Globalstar } \\
\text { M069 }\end{array}$} & TLE & 4/4 3:56 & 7790.9 & $7.12 \mathrm{E}-04$ & 51.958 & 335.137 & 98.175 \\
\hline & Double-r & $4 / 53: 41$ & 8226.3 & $5.63 \mathrm{E}-02$ & 52.262 & 334.625 & 314.544 \\
\hline & EKF & \multirow{2}{*}{ 4/6 4:29 } & 7653.3 & $9.53 \mathrm{E}-03$ & 52.409 & 335.698 & 127.909 \\
\hline & LS & & 7658.5 & $1.10 \mathrm{E}-03$ & 52.752 & 336.039 & 94.898 \\
\hline
\end{tabular}

The three satellites with addition image data needed to generate a refined orbit from OD methods are the M053, M056 and M069 Globalstar satellites. These satellites were 
chosen to determine that if the OD methods were to be used on a single pass consisting of multiple data points, they would be able to generate a comparably accurate model of the orbiting body to observations of the same satellite over multiple days.

The Globalstar M053 data consists of seven successive images during a single transit. Although the single trio-capture data derived from the initial orbital determination methods resulted in relatively large deviations from the initial TLE data, the addition of the consecutive trio-captures brought the eccentricity value down by an order of magnitude. The closer eccentricity approximation brought the semi-major axis to within a $0.5 \%$ error difference rather than the original $7.5 \%$ error difference from the initial TLE. This is a significant improvement that is reciprocated in the inclination and RAAN values as well.

The Globalstar M056 data is similar in trend but to a slightly lesser degree. Only five images along a single transit arc were used in the analysis of this satellite. The IOD error using the double-r method was roughly $11 \%$ off the initial TLE values. With the use of the EKF and LS OD methods, the error was reduced down to $1.3 \%$ and $1.7 \%$ respectively. A combination of the increased initial error seen in the IOD methods and the fewer number of data points utilized in the OD methods contributed to the increased error in the final values. This shows the advantageous effects of an increase in data points as well as the importance of minimalizing the inherent point error of the system being used.

The seven images used in the OD analysis of Globalstar M069 were taken over two separate nights. The first four images were captured on April $5^{\text {th }}, 2016$ and the final three images were captured on April $6^{\text {th }}, 2016$. The percent error between the initial orbit determination methods and the implementation of the EKF and LS methods are reduced from $5.6 \%$ error to a $1.7 \%$ error difference. Although the final error value is comparable to 
that seen from the Globalstar M056 data, the multi-day positional data experienced the least improvement after OD methods. This could be due to the combination of minute perturbations experienced over the period between observations and the variation in error based off the different telescope frame rotation angles for each night. Overall, the above values indicate a general increase in accuracy when using additional data points whether it be throughout a single orbital pass or from multiple passes.

Figure 5.1 is a graphical representation for an optimized telescope sizing for high apparent magnitude observation. This figure was produced by Brock Schmalzel for a slow temporal TLE error growth in observation of the $0.6 \times 0.6 \mathrm{~m}$ IMS-1 satellite.

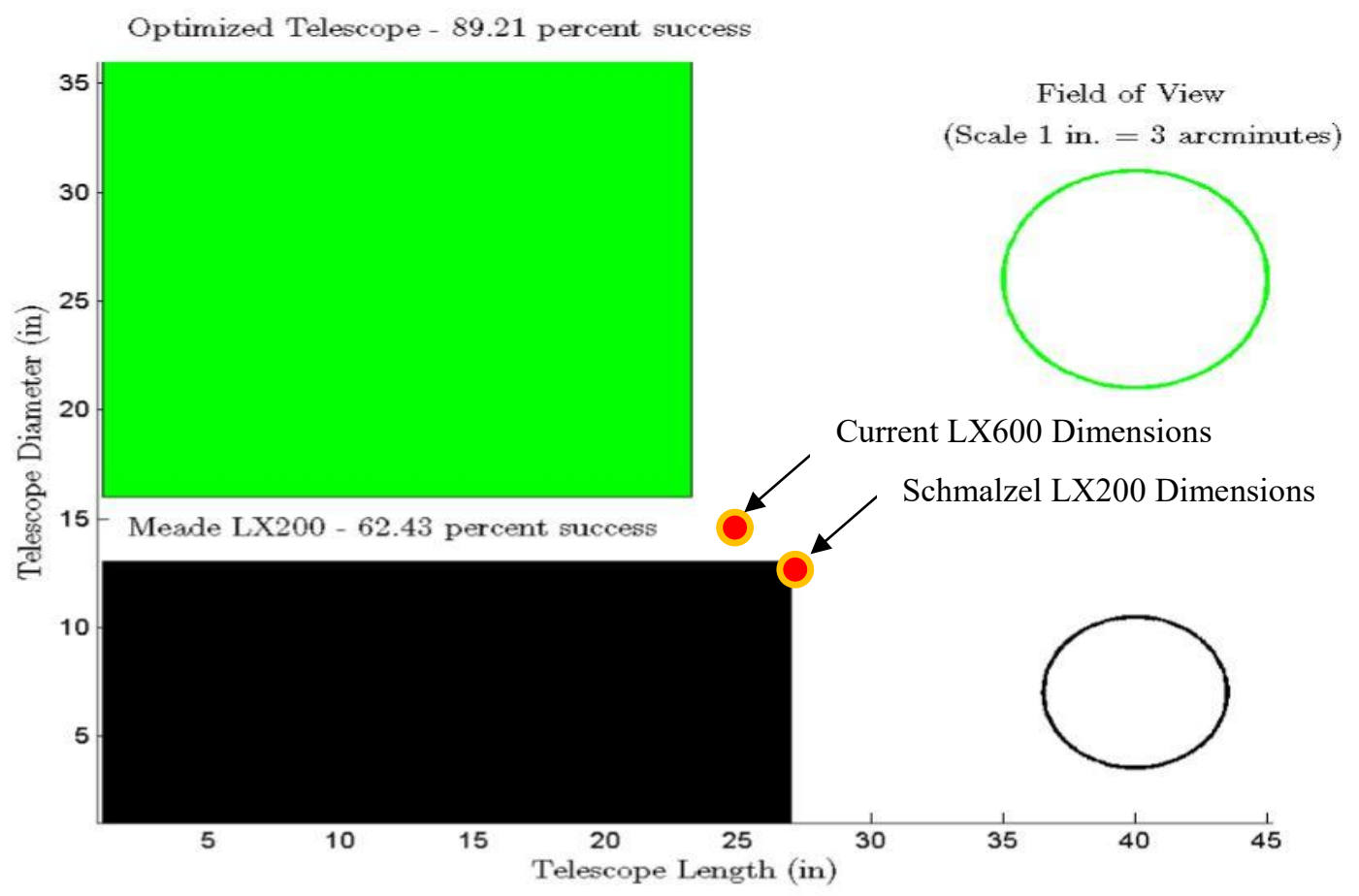

Figure 5.1: Slow Temporal Error Growth Telescope Optimization [23]

The calculations indicates that the 12" LX200 telescope used had a roughly $60 \%$ chance of imaging the passing object. With an optimized telescope consisting of a larger aperture diameter and shorter focal length, the probability of capturing a viable image 
increased to a nearly $90 \%$ success rate. The 14 " LX600 telescope used in the current data collection process was a step in the correct direction but still out of the range of the desired optimal aperture diameter and focal length. The increased aperture size and truncated focal length increase light sensitivity and FOV size which would prove to contribute considerably in the successful imaging of faint objects in young orbits.

Table 5.5 shows the mean Double-r percent error for the single trio-capture as well as the mean increase in the accuracy ( $\%$ change in error) once the Kalman filter and least squares methods are applied.

Table 5.5: Comparison of Mean Percent Error

\begin{tabular}{|c|c|c|c|c|c|c|}
\hline & Method & $a(\%)$ & e $(\%)$ & $i\left({ }^{\circ}\right)$ & $\Omega\left({ }^{\circ}\right)$ & $\omega\left(^{\circ}\right)$ \\
\hline \multirow{3}{*}{ 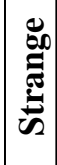 } & Double-r Error & $5.8 \pm 2.6$ & $7000 \pm 3000$ & $0.82 \pm 0.99$ & $0.69 \pm 4.19$ & $211.7 \pm 40.7$ \\
\hline & EKF Improvement & $6.4 \pm 4.0$ & $5700 \pm 600$ & $0.52 \pm 0.21$ & $0.18 \pm 0.62$ & $167.0 \pm 100.3$ \\
\hline & LS Improvement & $6.1 \pm 3.9$ & $5500 \pm 800$ & $0.79 \pm 0.44$ & $0.07 \pm 0.72$ & $69.2 \pm 47.4$ \\
\hline \multirow{3}{*}{ 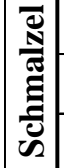 } & Double-r Error & $6.2 \pm 3.7$ & $19000 \pm 18000$ & $0.21 \pm 0.18$ & $3.0 \pm 2.1$ & $73.0 \pm 55.0$ \\
\hline & EKF Improvement & $5.8 \pm 5.0$ & $29000 \pm 3000$ & $0.27 \pm 0.24$ & $0.06 \pm 0.07$ & $44.0 \pm 25.4$ \\
\hline & LS Improvement & $6.3 \pm 4.2$ & $28000 \pm 19000$ & $0.04 \pm 0.17$ & $-0.47 \pm 0.30$ & $-23.6 \pm 104.9$ \\
\hline
\end{tabular}

There are large percentage errors between the initial TLE data and the IOD orbit propagations, specifically in the eccentricity values. The potential root cause of these errors lie in the relatively short arc captured in some of the observations. Over the period of a single satellite transit, a range of only $6.5 \%$ to $9.5 \%$ of the arc is observed. Although a series of images were taken from a single transit, it still constitutes only a small portion of the whole. Such a stark contrast in error decrease is likely caused by the use of data from only the single transit in relation to the multi-night images captured in Brock Schmalzel's data. The intent was not to achieve a dramatic increase in accuracy, but to 
attain a comparable accuracy when only using data from a single orbit transit to that of multiple nights of observations.

The decrease in error percentage of the initial Double-r data can be contributed to the increased resolution of the CCD camera. With the more concise pixel value along the transit path, the arc of the satellite can be initially refined to a more precise propagation. The percent change in error after the use of the EKF and LS values show a slight improvement in accuracy compared to Schmalzel's data. Whether this is due to the increase in CCD pixel resolution or the more well-defined arc length of a single transit is unclear. The EKF values seem to present a slight advantage in refining the satellite orbit to a higher degree of accuracy than the LS method, but a definitive advantage is still inconclusive based on the data accumulated. Any additional data sets of each satellite from subsequent orbital passes would likely continue to increase the accuracy of the orbital elements.

Since some of the initial observational data yielded large initial eccentricity values, a final attempt to improve the orbital determination results was to implement the MATLAB fmincon function in order to effectively circularize the orbit by forcing the eccentricity to diminish to orders of magnitude smaller.

\section{Table 5.6: Fmincon Optimization of Globalstar M026 Transit}

\begin{tabular}{|c|c|c|c|c|c|}
\hline Souce & $\boldsymbol{a}(\mathbf{k m})$ & $\mathbf{e}$ & $\boldsymbol{i}\left(^{\circ}\right)$ & $\boldsymbol{\Omega}\left(^{\circ}\right)$ & $\boldsymbol{\omega}\left(^{\circ}\right)$ \\
\hline Original Det. & 11008.5 & 0.651 & 49.569 & 109.441 & 138.807 \\
\hline Optimized & 6729.7 & $2.28 \mathrm{E}-07$ & 49.570 & 109.443 & 344.628 \\
\hline TLE & 7788.7 & $6.37 \mathrm{E}-04$ & 51.956 & 334.714 & 51.590 \\
\hline
\end{tabular}

By constraining the optimizer to maintain angular momentum to within $10 \%$ of the initial input values and optimizing for the minimum eccentricity, a much more accurate set 
of COEs can be generated [23]. This process is predicated upon the assumption that the observed satellite orbits are roughly circular. An example of the fmincon optimization can be seen in Table 5.6. The observational data used in the above example was an extreme case of a small arc trio-capture in order to demonstrate the greatly improved outcome from such a simple eccentricity optimization derived from such a drastically eccentric initial orbit determination. The fmincon optimizer was used only as an exercise in determining if a comparably accurate solution can be produced from the circularization of the orbit. The fmincon optimized data was not used in the calculation of IOD and OD values. This test was solely to determine if satellite observations with large initial errors could be rendered useful if the circular orbit assumption was forced. This option may prove useful in the initial attainment of an ill-defined or largely perturbed orbit.

\subsection{TLE Positional Error}

After the optimization of the useful data sets, the positional error of each of the observed satellites are reasonably close to the predicted values generated from the STK software of previously observed data points. The CTE and ATE for each of the data sets

can be seen in Table 5.7. The remaining CTE and ATE data can be found in Table C.2 in Appendix C. 
Table 5.7: Cross-Track and Along-Track Positional Error of Satellite Trajectories

\begin{tabular}{|c|c|c|c|c|c|c|c|c|}
\hline Sat ID & Target RA ( $\left.{ }^{\circ}\right)$ & Det. RA $\left({ }^{\circ}\right)$ & $\triangle \mathrm{RA}\left({ }^{\circ}\right)$ & Target DEC $\left({ }^{\circ}\right)$ & Det. DEC $\left({ }^{\circ}\right)$ & $\triangle \mathrm{DEC}\left({ }^{\circ}\right)$ & CTE (km) & ATE (km) \\
\hline GS M026 & 158.88539 & 158.86360 & $2.18 \mathrm{E}-02$ & 8.46537 & 8.75550 & $2.90 \mathrm{E}-01$ & 0.0467 & 0.0102 \\
\hline GS M026 & 183.17446 & 183.05540 & 1.19E-01 & 25.78285 & 25.91330 & $1.30 \mathrm{E}-01$ & 0.0525 & 0.0210 \\
\hline GS M034 & 116.78565 & 116.51260 & $2.73 \mathrm{E}-01$ & 18.48398 & 18.49130 & 7.32E-03 & 0.0251 & 0.0120 \\
\hline GS M036 & 87.32625 & 87.10850 & $2.18 \mathrm{E}-01$ & 5.76384 & 5.82580 & $6.20 \mathrm{E}-02$ & 0.1930 & 0.0240 \\
\hline GS M036 & 105.75577 & 105.75150 & 4.27E-03 & 34.93953 & 35.23090 & 2.91E-01 & 0.1929 & 0.0129 \\
\hline GS M053 & 171.43180 & 171.29460 & $1.37 \mathrm{E}-01$ & 11.29348 & 11.49050 & $1.97 \mathrm{E}-01$ & 0.0996 & 0.0213 \\
\hline GS M053 & 188.35817 & 188.20300 & $1.55 \mathrm{E}-01$ & 23.15474 & 23.29560 & $1.41 \mathrm{E}-01$ & 0.0447 & 0.0214 \\
\hline GS M053 & 210.70084 & 210.62670 & $7.41 \mathrm{E}-02$ & 32.42395 & 32.49370 & $6.98 \mathrm{E}-02$ & 0.1979 & 0.0107 \\
\hline GS M056 & 83.56312 & 83.34310 & $2.20 \mathrm{E}-01$ & 1.83488 & 1.90130 & $6.64 \mathrm{E}-02$ & 0.1486 & 0.0212 \\
\hline GS M056 & 90.16850 & 90.36110 & $1.93 \mathrm{E}-01$ & 10.00828 & 10.22240 & 2.14E-01 & 0.0568 & 0.0133 \\
\hline GS M056 & 97.33471 & 97.00650 & $3.28 \mathrm{E}-01$ & 18.23550 & 18.21600 & $1.95 \mathrm{E}-02$ & 0.0941 & 0.0219 \\
\hline
\end{tabular}

The CTE and ATE values have a slight variance from their respective means with exception of 11 outliers within the 47 data points in the cross-track direction. Only one of the outliers are depicted in Fig. 5.1 in order to better visualize the scale of the remaining data. The outliers deviated from the mean on an average of $31 \mathrm{~km}$ with the maximum being $61 \mathrm{~km}$. All of the outliers had a commonality of a negative declination observation angle which deviated from the target declination by up to 2 degrees in some cases.
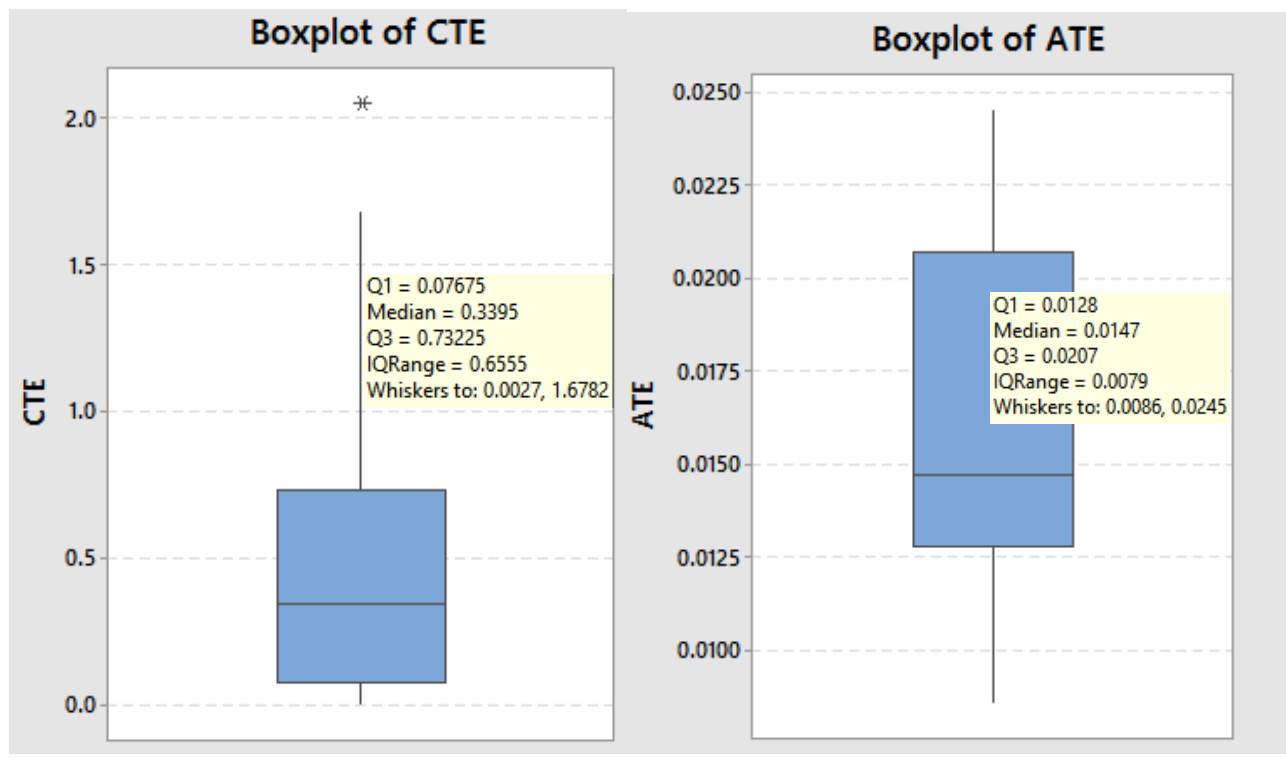

Figure 5.2: Along-Track Error and Cross-Track Error Boxplots

The occurrence of such a regularized pattern of error seems to suggest a decrease in accuracy based on an orientation that requires a declination value below -10 degrees. 
This is most likely due to compounding error from an increased frame rotation angle. When disregarding the outliers, the median CTE was $0.339 \mathrm{~km}$ with an interquartile range (IQR) of $0.656 \mathrm{~km}$, a first quartile $(\mathrm{Q} 1)$ of $0.077 \mathrm{~km}$, and a third quartile (Q3) value of $0.732 \mathrm{~km}$. This shows the distribution of data points within the CTE being fairly densely populated, indicating a fairly low variability across the multiple observations.

The median value of the ATE is $0.015 \mathrm{~km}$ with an IQR of $0.008 \mathrm{~km}, \mathrm{Q} 1$ of 0.013 $\mathrm{km}$, and Q3 of $0.021 \mathrm{~km}$. The ATE values are noticeably smaller than their CTE counterparts. The reasoning behind this gap could be due to the refinement of the satellite TLEs over the years they have been in orbit. Globalstar satellite position and velocity vectors are observed regularly and are constantly updated. Since the ATE values are based solely on the computer clock error, the relatively large frame rotation error based off of the satellite pixel uncertainties would have a more significant affect than the TLE data associated to the computer's internal clock. Although the observed satellites were fairly accurate in the along-track direction, this would not be the case for newly deployed CubeSats. The lack of reliable initial TLE data and the errors associated with atmospheric perturbations in the along-track direction would increase the initial ATE values considerably. The Globalstar ATE values are dependent on the accuracy of the orbit propagations provided by the STK software. However, provided with a given initial observation and TLE estimate, with every compounding observation the orbit can be refined to a higher and higher degree of accuracy. 


\subsection{Imaging Methods}

When attempting to make initial observations, the procedure developed by Brock Schmalzel was implemented in order to become familiarized with the process of satellite imaging. However, there were a few fundamental differences in the hardware used which subsequently led to the alterations in the imaging process. In this section, the changes in hardware, their effects on the data collected, and the reasons for utilizing different observation methods will be discussed. After taking multiple observations over the span of months, both pros and cons were found within the observational procedure used in this thesis as well as that used by Brock Schmalzel. This section is designed to help future observers in determining which process would best fit the needs of their desired data based off of both separate procedural constraints.

Besides the previously mentioned difference in telescope size used in Brock Schmalzel's thesis, the most prevalent difference in hardware is the camera. Schmalzel used the Lumenera SKYnyx2-0 monochrome camera with a resolution of 640x480 pixels shooting at 60 frames per second while I alternatively used a SBIG ST-10XME CCD Camera with a $2184 \times 1472$ pixel resolution. Even with the $2 \mathrm{X} 2$ binning scheme, the resolution of the SBIG CCD is superior and in itself was a leading reason for the use of the SBIG CCD. Despite the resolution difference, the main cause for the procedural difference is due to the desired data sets of a single satellite per transit. 


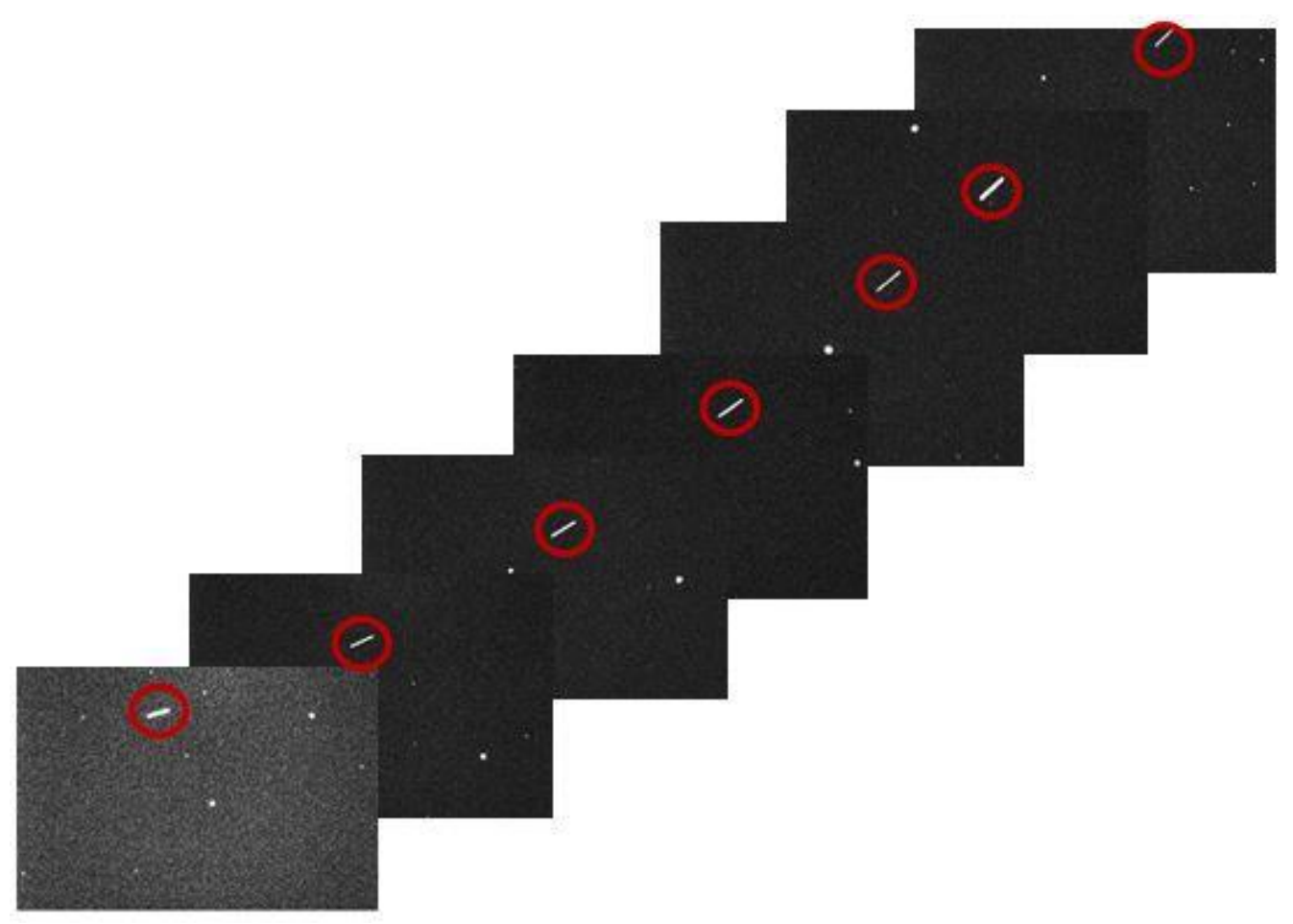

Figure 5.3: Series of Seven Images of Globalstar M053 in Single Pass

As seen in Figure 5.3, up to 7 consecutive images of a satellite along a single continuous transit was able to be captured. This is due to both the change in camera hardware as well as the procedural change from a "point and wait" method which was implemented by Brock Schmalzel to a "point and shoot" method.

The differences in these methods are first and foremost facilitated by the type of camera. Since Schmalzel's camera recorded at $60 \mathrm{fps}$, he was able to point the telescope in a desired location in the night sky and record until a transit occurred in the allotted time period. The camera recorded for \pm 30 seconds around the previously determine transit time in order to ensure an image. While this "point and wait" method does ensure a high success rate, it uses up a precious window of observation time for each satellite transit. 
The "point and shoot" method alternatively requires a relatively high precision TLE in order to accurately image the desired satellite. The "point and shoot" method required the observer to map out a series of timestamps along the predicted satellite transit path and dither the telescope to the next RA and DEC location between each exposure. Due to the limited observable arc and the speed at which the LEO satellites travel, only a single 0.2 second exposure image is taken with a $\sim 4$ second image download time. These two methods differ dramatically in practice and both have pros and cons associated with them.

Schmalzel self-admittedly experienced issues with the computer terminal's internal clock and could not reliably image passing satellites without taking 60fps for 60 seconds surrounding the estimated transit time, totaling roughly 3600 images per pass. These images were then manually searched through until the object was found and the correct time stamp cataloged [23]. This can be a tedious process and creates a large amount of unusable data. However if an initial TLE is ill-defined, the \pm 30 seconds of observational data of a predicted orbit in the along-track direction can be extremely valuable and raise your chance of a successful image significantly.

The "point and shoot" method implemented in this thesis required well-defined initial TLEs in order to successfully image the satellite. Although this method required a higher fidelity TLE, there is a dramatic decrease in the imaging time and a higher potential yield in useful data per orbit. While a series of satellite images were able to be captured within a single orbit, it is now apparent that the "point and wait" method would be more suitable for the observation of nanosatellites. Due to the large atmospheric drag of the low orbits adding to the ATE uncertainties and the possibly ill-defined initial TLEs, it would be more advantageous to start initial nanosatellite observation with the "point and wait" 
method. Once a nanosatellite has been observed and a TLE is reliably refined, the "point and shoot" method would allow for much more data points to even further refine the satellite's orbital propagation. A full procedural overview of the "point and shoot" method can be found in Appendix D while Brock Schmalzel's "point and wait" procedure is referenced in the bibliography [23].

\subsection{Causes of Error}

The fidelity of the data collected in this thesis could be attributed to instrumentation errors as well as pixel uncertainty estimations near the points of interest within the images. The error values associated with the instrumentation may have been overly generalized to account for the fluctuations in the mean error from each night of observation. Although the instrumentation pointing and focus were calibrated each night of observation, the sometimes wild inconsistencies in accuracy and clarity over the span of the night required recalibration.

An additional reason for limited data accuracy within the process is due to the $2 \mathrm{X} 2$ binning scheme used in data collection. Since the satellite's visible arc is an average of 6 minutes, there is a small window of opportunity to acquire multiple images in one pass. With the slewing of the telescope, orientation on a background star, resyncing the telescope

and capturing the image, there is very little time to waste. Although the $2 \mathrm{X} 2$ binning increased the visibility of the passing satellites, it decreased the resolution. For a more accurate result, it is suggested to utilize the standard $1 \mathrm{X} 1$ binning scheme. 
The third cause of error and the most drastic loss of usable data was from the attempt to utilize the starting and ending data points within a single image as well as a third data point from a separate image. The 0.2 exposure time only allowed for a differential distance of roughly 1 arc minute. The minimal arc distance was too small to accurately converge on a comparable orbital path and rendered all satellite observations with only two captured images unusable. For future consideration, it is highly recommended to obtain three independent images of each observed satellite. 


\section{CONCLUSION}

As the availability of amateur telescopes continue to rise, the demonstration of their capabilities in unique situations will prove their validity in implementation of satellite tracking. Although larger observatories can eventually acquire more accurate TLEs, they are not as readily available in determining the orbits of lower priority items within the first month after launch due to the increasing surplus of satellites and orbital debris. With the ability and the interest in observing specified nanosatellites, the Cal Poly observatory has the photometric sensitivity and pointing tolerances to increase the accuracy of the TLE within the short time after launch.

Alternatively, the implementation of multiple amateur sized telescopes have the potential to be a valuable alternative in satellite orbital determination for larger satellites as well. With the relative ease of imaging the Globalstar satellites, it would be reasonable to assign a series of amateur telescopes to track the larger satellites and therefore take the excessive load off of larger observatories. This would in turn allow the larger observatories to more readily update the TLEs of smaller objects such as nanosatellites and orbital debris. The increased aperture sizes and focal ratios would be conducive to imaging smaller 
orbiting bodies. The unceasing increase in satellite launches and unforeseen causes of additional orbital debris require a response in keeping track of the growing population. With the increased data sets acquired from continuous tracking of low-cost amateur telescopes, they could be the most accurate and cost-effective solution to this growing problem.

\subsection{Recommendations for Future Work}

This section is comprised of suggestions for future research topics that would build upon the material covered in this thesis. Any of the proposed topics could serve as a basis for further exploration into the advancement of orbital determination of Cal Poly launched CubeSats.

\section{Optical Fence}

Alternatively to the instrumentation used at the Cal Poly observatory, the use of an optical fence can increase the viewable trajectory of an orbiting object. An optical fence is a series of telescopes used in tandem across large distances that can be used to observe longer arcs of a satellite as they pass overhead. Observations along the increased path length can potentially lead to more accurate orbital determination in a shorter amount of time. Additionally, if the telescope's FOVs could be aligned side-by-side, perpendicular to the satellite's projected orbital path, a greater likelihood of acquiring an initial reading of a poorly defined TLE would be possible. This process would likely yield the best results in developing a reliable TLE within the first few weeks after a CubeSat launch by 
accounting for a larger CTE. It is recommended to implement the use of the Lumenera SKYnyx2-0 monochrome astrophotography camera in order to take a continuous stream of images to account for the ATE that may also be encountered.

Dr. Keller of the Cal Poly Physics Department operates an optical fence that can potentially be utilized for analysis on the accuracy differences between the standard single telescope and the optical fence. The MATLAB code scopefence.m was developed to provide an n-amount of RA/DEC target angles for an n-amount of telescopes used within the optical fence.

\section{Data analysis for increased accuracy for compounding trio-captures using EKF}

The OD methods used in this thesis were limited to only a second set of triocaptures for data refinement for multiple satellites. An interesting alternative might be the use of EKF for multiple trio-captures on a single orbiting body and determining the statistical improvement in the orbital elements or CTE and ATE values for each subsequent iteration. The further use of the fmincon function in optimization of the orbits based on constraints of different COE values may prove to increase accuracy as well.

\section{Photometric Analysis of Satellite Albedo}

Due to the defining albedo characteristics of the materials surrounding each

Opticube CubeSats, a different reflective signatures can be observed. The amount of reflected light from the surface of the CubeSats can be attributed to a specific satellite. 
Since most CubeSats are launched in groups, the exact placement of each satellite is not specifically known until weeks after deployment.

If the imaging of passing CubeSats either through the use of the Cal Poly observatory or the optical fence becomes well-documented, a photometric analysis of the data could be used to verify the satellite's identification. In order for this thesis topic to be viable, the specifications on the satellite's albedo must be fully understood prior to launch, the effects of satellite tumbling on the apparent brightness of the object must be considered, and all other causes of photometric dampening prior to launch. This topic would be valuable in the ability to accurately determine a CubeSat's identity well before they are accurately updated to larger databases.

\section{Acquisition Targeting Orbital Determination}

The current tracking system being utilized by Cal Poly for the communication of

CubeSats is through Acquisition and Tracking techniques. This is an essential operation in communication with passing satellites in which the communication link is established through a directional antenna located on Cal Poly's campus. The initial orbit of the object must first be well known in order to point the antenna in the correct location and track at the correct rate. Since the CubeSats are at such a low altitude, the tracking rate is extremely fast which makes it more difficult to establish a link in a shortened time frame.

Although the communication antenna is used to downlink data and uplink various commands, it has not been used for orbital determination data. Using this antenna, the solar phase angle of the satellites are not relevant and a larger path can be observed for each 
pass. Depending on how accurately the antenna pointing is defined and the ability to track the satellites motion, it seems feasible to use the pointing coordinates to determine its orbit.

In the first few months after a CubeSat launch when the initial TLE is not very accurate, the directional antenna could use a larger voltage output to ensure a communication link with the satellite. Based on the SNR encountered with each communication link, it may be possible to determine a location within the antennas link radius. As the quantity of communication links increase, more pointing data is collected and the accuracy of the satellite's position may be able to be refined.

\section{StarLock Implementation}

Although the StarLock system was not utilized in the observations taken, it may prove to be useful in the observation of nanosatellites. Due to the nanosatellites being very dim, the active tracking with StarLock would allow for a larger exposure time amplifying the amount of gathered light. The feasibility of this prospect is unknown due to the physical tracking speed constraints of the hardware. Although there would be a higher change for the satellite to be visible, an accurate initial TLE will be required to track the satellite. 


\section{BIBLIOGRAPHY}

[1] A. Mammano. Observations of Artificial Satellites. In Traditional Technical Scientific Meeting on Space, number 19-23, Rome, Italy, 1962.

[2] "Classical Orbital Elements." Http://satelliteorbitdetermination.com. N.p., n.d. Web. Winter 2016.

[3] Coder, Ryan D., and Marcus J. Holzinger. Sizing of a Raven-class Telescope Using Performance Sensitivities Ryan D. Coder (n.d.): n. pag. Georgia Institute of Technology. Web.

[4] "Compute Altitude and Azimuth Using Either Quaternions or Rotation Matrix or Roll, Pitch and Yaw Component." Matrices. N.p., 28 Apr. 2011. Web. 2 Feb. 2016.

[5] "Definition of Two-line Element Set Coordinate System." Human Space Flight (HSF) - Realtime Data. N.p., 23 Sept. 2011. Web. Winter 2016.

[6] Di Cicco, Dennis. "Of Pixel Size and Focal Reducers." Sky \& Telescope. N.p., 17 July 2006. Web. 17 Oct. 2015.

[7] D. Scott Birney and D. Oesper. Observational Astronomy. Cambridge University Pres, New York, second edition, 2006. 
[8] Foley, Justin. CubeSat, California Polytechnic State University, 2016. Email.

[9] Globalstar.com. Globalstar, 2016. Web.

[10] H.D. Curtis. Orbital Mechanics for Engineering Students. Elsevier, Burlington, MA, second edition, 2010.

[11] "High Quantum Efficiency CCD Cameras." ST-10 Information. Santa Barbara Instrument Group, Inc., 8 June 2010. Web. 17 Mar. 2016.

[12] Houston, Heather. "Orbit Determination for Scientific Missions." Colorado Center of Astrodynamics Research, 2013. Web. 19 Jan. 2016.

[13] Kervin, Paul W., Doyle Hall, Mark Bolden, and Joe Toth. Phase Angle: What Is It Good For? Amostech.com. Air Force Research Laboratory, n.d. Web.

[14] "LX600-ACF 14" F/8." Http://www.meade.com/products/telescopes. Meade Instruments Corp., 2014. Web.

[15] McCarthy, John, PhD. "Astronomical Coordinate Systems." - Quora. N.p., 25 Sept. 2015. Web.

[16] N2YO ITPROSTAR. Real Time Satellite Tracking. http://www.n2yo.com/, 2016.

[17] Neets. "Satellite Acquisition and Targeting." 17-Radio-Frequency Communications Principles, n.d. Web.

[18] Nishimoto, Daron, David Archambeault, Paul Kervin, and David Gerwe. "Satellite Attitude from a Raven Class Telescope." (2010): n. pag.

[19] "PolySat." PolySat. N.p., n.d. Web. 27 Jan. 2016. 
[20] Poole, Ian. "Signal to Noise Ratio, SNR." Radio-Electronics.com. Adrio Communications Ltd., n.d. Web.

[21] Richmond, Michael. "Differential Photometry." N.p., 2006. Web. 06 Jan. 2016.

[22] Schaeperkoetter, Andrew V. "A Comprehensive Comparison between AnglesOnly Initial Orbit Determination Techniques." Thesis. Texas A\&M University, 2011. Web.

[23] Schmalzel, Brock. The Feasibility and Application of Observing Small LEO Satellites with Amateur Telescopes. Thesis. California Polytechnic State University, 2013. N.p.: n.p., n.d. Print.

[24] Sellers, Jerry Jon. "Astro Primer: SGP-4." Astro Primer: SGP-4. N.p., n.d. Web. 19 Feb. 2016.

[25] "SKYnyx2-0." Lumenera.com. Lumenera Corporation, 2016. Web.

[26] Space Track Satellite Situation Report, JFCC SPACE, Vandenberg AFB, CA. http://www.space-track.org.

[27] Tapley, Byron D., Bob Schultz, and George H. Born. Statistical Orbit Determination. San Diego: Elsevier Science Co, 2004. Print.

[28] "TCF-S Temperature Compensating Focuser." Optec. Optec, Inc., 2013. Web. 17 Mar. 2016.

[29] "TheSkyX Professional and Serious Astronomer Edition User Guide." Software Bisque, Inc., Nov. 2015. Web. 
[30] Vallado, David A., and Agapov, Vladimir. "Orbit Determination Results From Optical Measurements". Centerforspace.com. European Space Astronomy Centre, 3 May 2010.

[31] Vallado, David A., and Paul Crawford. SGP4 Orbit Determination.Centerforspace.com. American Institute of Aeronautics and Astronautics, n.d. Web.

[32] Vallado, David A., and Wayne D. McClain. Fundamentals of Astrodynamics and Applications. Dordrecht: Kluwer Academic, 2001. Print.

[33] Vetter, Jerome R. "Fifty Years of Orbital Determination: Development of Modern Aerodynamic Methods." Fifty Years of Orbit Determination: Development of Modern Astrodynamics Methods (n.d.): n. pag. Jhualp.edu. 2007. Web.

[34] "Measuring Instrumental Magnitudes." The CCD Photometric Calibration Cookbook. Council for the Central Laboratory of the Research Councils, 2001.

[35] J. B. M. Mark R. Ackermann, John T. McGraw and P. C. Zimmer. Blind Search for Micro Satellites in LEO: Optical Signatures and Search Strategies In AMOS Technical Conference, Sandia National Laboratories, Albuquerque, NM, September 2003.Web. 


\section{Appendix A SELECTED IMAGES AND RAW DATA}

The images within this appendix were selected to illustrate certain aspects of the observation and/or imaging process defined within the figure title. All images were taken with identical filters, exposures, and binning scheme.

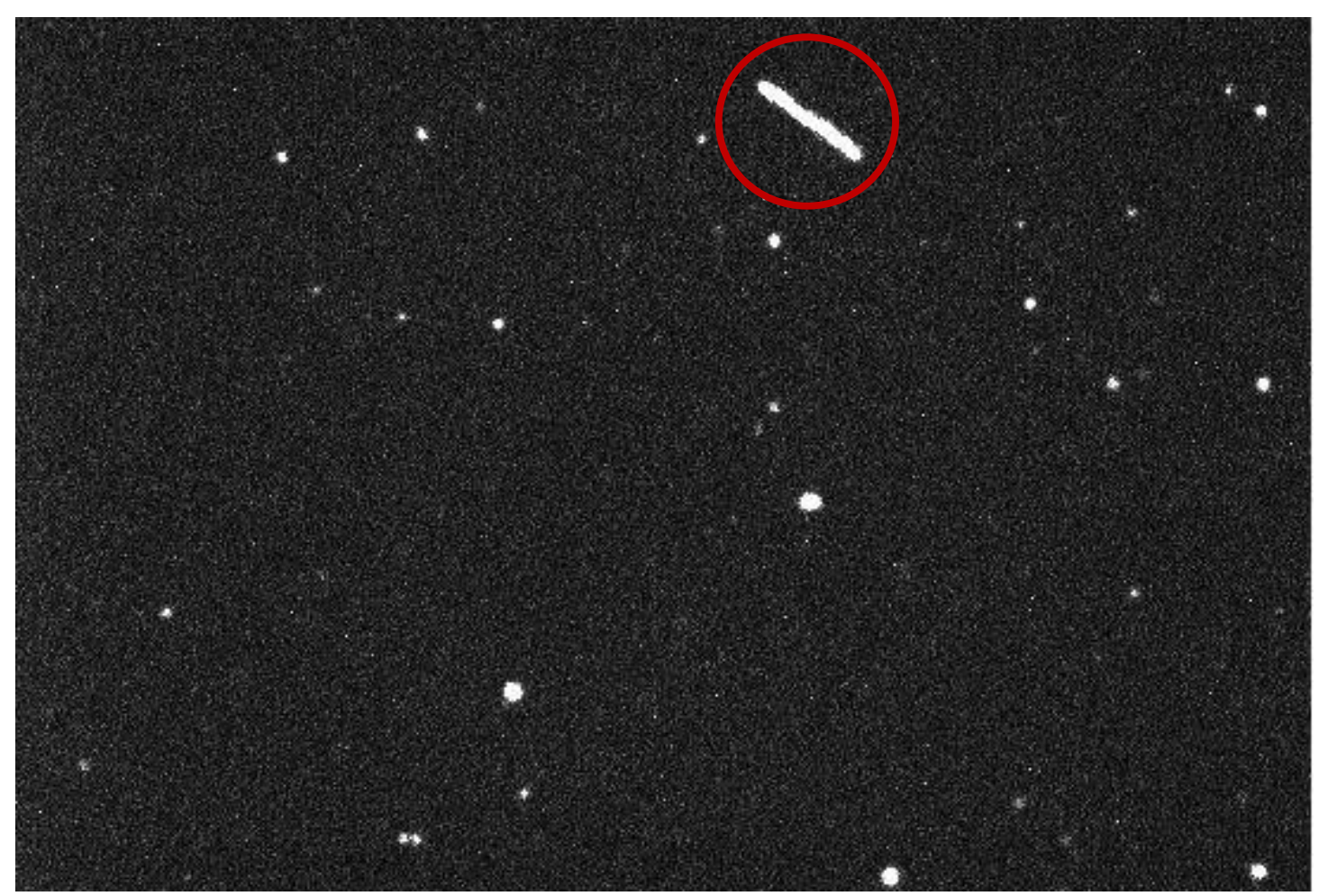

Figure A.1: Image taken of Globalstar M037, on April 5 ${ }^{\text {th }}$, 2016, at 9:19 PM PST. An example of a highly reflective instance. 
Table A.1: All observations positional data in both equatorial and horizontal coordinate systems with time stamps in UTC

\begin{tabular}{|c|c|c|c|c|c|}
\hline Sat ID & $\mathbf{A z}\left({ }^{\circ}\right)$ & El $\left({ }^{\circ}\right)$ & Time UTC & $\mathbf{R A}\left({ }^{\circ}\right)$ & $\operatorname{DEC}\left({ }^{\circ}\right)$ \\
\hline Globalstar M001 & 191.001 & 30.028 & 2016/03/16 03:42:17.170 & 98.670 & -23.856 \\
\hline Globalstar M001 & 176.276 & 42.959 & 2016/03/16 03:44:15.300 & 112.353 & -11.667 \\
\hline Globalstar M001 & 142.986 & 54.409 & 2016/03/16 03:46:19.120 & 130.687 & 5.200 \\
\hline Globalstar M026 & 146.429 & 59.138 & 2016/04/02 04:47:39.270 & 158.885 & 8.465 \\
\hline Globalstar M026 & 94.004 & 54.143 & 2016/04/02 04:49:37.619 & 183.174 & 25.783 \\
\hline Globalstar M030 & 197.123 & 32.643 & 2016/03/16 03:25:43.100 & 89.608 & -20.186 \\
\hline Globalstar M030 & 188.558 & 27.553 & 2016/03/16 03:27:01.919 & 96.763 & -26.629 \\
\hline Globalstar M030 & 183.493 & 23.744 & 2016/03/16 03:28:00.730 & 101.772 & -30.863 \\
\hline Globalstar M034 & 229.878 & 66.245 & 2016/04/06 04:06:04.160 & 116.786 & 18.484 \\
\hline Globalstar M034 & 233.392 & 77.258 & 2016/04/06 04:07:00.040 & 124.495 & 27.147 \\
\hline Globalstar M036 & 245.674 & 38.83 & 2016/04/06 03:54:31.440 & 87.326 & 5.764 \\
\hline Globalstar M036 & 257.114 & 55.259 & 2016/04/06 03:56:32.210 & 96.604 & 21.785 \\
\hline Globalstar M036 & 277.256 & 67.227 & 2016/04/06 03:57:53.100 & 105.756 & 34.940 \\
\hline Globalstar M037 & 278.998 & 25.984 & 2016/04/06 04:13:33.039 & 64.902 & 21.587 \\
\hline Globalstar M037 & 266.601 & 31.883 & 2016/04/06 04:14:56.039 & 76.458 & 15.315 \\
\hline Globalstar M037 & 212.012 & 34.662 & 2016/04/06 04:19:03.279 & 112.300 & -13.920 \\
\hline Globalstar M039 & 270.166 & 31.954 & 2016/04/02 03:15:11.130 & 55.943 & 929 \\
\hline Globalstar M039 & 279.471 & 38.851 & 2016/04/02 03:16:15.839 & 58.994 & 27.845 \\
\hline Globalstar M039 & 299.534 & 47.657 & $2016 / 04 / c$ & 741 & .274 \\
\hline Globalstar M050 & 296.921 & 44.674 & 2016/04/05 03:53:52.369 & 73.142 & 41.993 \\
\hline Globalstar M050 & 252.189 & 66.17 & 2016/04/06 03:47:31.140 & 105.902 & 25.324 \\
\hline Globalstar M050 & 212.694 & 67.187 & 2016/04/06 03:48:41.429 & 118.831 & 15.447 \\
\hline Globalstar M053 & 158.566 & 47.439 & 2016/04/02 04:35:42.520 & 153.591 & -5.061 \\
\hline Globalstar M053 & 143.381 & 51.378 & 2016/04/02 04:36:53.820 & 161.400 & 2.442 \\
\hline Globalstar M053 & 122.185 & 52.667 & 2016/04/02 04:38:17.600 & 171.432 & 11.293 \\
\hline Globalstar M053 & 104.816 & 50.452 & 2016/04/02 04:39:28.839 & 180.563 & 18.222 \\
\hline Globalstar M053 & 92.934 & 46.877 & 2016/04/02 04:40:27.589 & 188.358 & 23.155 \\
\hline Globalstar M053 & 80.534 & 40.584 & 2016/04/02 04:41:49.409 & 199.273 & 28.546 \\
\hline Globalstar M053 & 71.326 & 33.387 & 2016/04/02 04:43:18.230 & 210.701 & 32.424 \\
\hline Globalstar M056 & 221.154 & 28.248 & 2016/03/17 03:28:22.649 & 69.583 & -15.535 \\
\hline Globalstar M056 & 220.128 & 39.545 & 2016/03/17 03:30:00.650 & 76.970 & -6.505 \\
\hline Globalstar M056 & 218.653 & 49.936 & 2016/03/17 03:31:11.609 & 83.563 & 1.835 \\
\hline Globalstar M056 & 216.209 & 60.174 & 2016/03/17 03:32:10.080 & 90.168 & 10.008 \\
\hline Globalstar M056 & 211.106 & 70.599 & 2016/03/17 03:33:02.569 & 97.335 & 18.236 \\
\hline Globalstar M058 & 257.664 & 32.356 & 2016/04/02 04:13:26.949 & 76.893 & 9.321 \\
\hline Globalstar M058 & 266.427 & 42.088 & 2016/04/02 04:14:58.609 & 81.790 & 20.461 \\
\hline Globalstar M058 & 277.905 & 50.407 & 2016/04/02 04:16:11.029 & 86.822 & 31.120 \\
\hline Globalstar M062 & 258.977 & 53.335 & 2016/03/16 03:54:00.330 & 72.892 & 21.737 \\
\hline Globalstar M062 & 229.829 & 57.077 & 2016/03/16 03:55:16.309 & 87.259 & 11.473 \\
\hline Globalstar M062 & 170.913 & 34.964 & 2016/03/16 03:59:08.269 & 121.180 & -19.226 \\
\hline Globalstar M069 & 287.623 & 39.828 & 2016/04/05 03:41:22.719 & 66.512 & 34.046 \\
\hline Globalstar M069 & 274.889 & 49.279 & 2016/04/05 03:42:33.000 & 80.913 & 28.903 \\
\hline
\end{tabular}

Continued... 


\begin{tabular}{|l|c|c|c|c|c|}
\hline Sat ID & $\mathbf{A z}\left({ }^{\circ}\right)$ & El $\left({ }^{\circ}\right)$ & Time UTC & RA $\left(^{\circ}\right)$ & DEC $\left({ }^{\circ}\right)$ \\
\hline Globalstar M069 & 253.17 & 57.166 & $2016 / 04 / 0503: 43: 41.020$ & 95.383 & 20.942 \\
\hline Globalstar M069 & 232.03 & 59.487 & $2016 / 04 / 0503: 44: 27.719$ & 104.964 & 14.057 \\
\hline Globalstar M069 & 271.562 & 21.771 & $2016 / 04 / 0604: 25: 47.109$ & 67.921 & 13.591 \\
\hline Globalstar M069 & 261.431 & 25.109 & $2016 / 04 / 0604: 26: 57.850$ & 76.327 & 7.764 \\
\hline Globalstar M069 & 237.734 & 28.173 & $2016 / 04 / 0604: 29: 13.089$ & 92.946 & -6.387 \\
\hline Globalstar M075 & 240.081 & 41.467 & $2016 / 04 / 0504: 13: 58.810$ & 96.083 & 4.452 \\
\hline Globalstar M081 & 215.642 & 49.696 & $2016 / 03 / 1703: 24: 02.439$ & 83.343 & 0.668 \\
\hline Globalstar M081 & 209.133 & 64.741 & $2016 / 03 / 1703: 25: 14.109$ & 93.498 & 12.616 \\
\hline Globalstar M089 & 237.347 & 43.522 & $2016 / 04 / 0204: 58: 04.240$ & 107.068 & 4.510 \\
\hline Globalstar M089 & 201.958 & 38.255 & $2016 / 04 / 0205: 00: 19.640$ & 127.805 & -13.686 \\
\hline Globalstar M094 & 268.598 & 23.696 & $2016 / 04 / 0504: 40: 37.000$ & 73.841 & 12.354 \\
\hline Globalstar M094 & 256.275 & 27.173 & $2016 / 04 / 0504: 41: 55.810$ & 83.526 & 5.257 \\
\hline Globalstar M094 & 242.776 & 28.941 & $2016 / 04 / 0504: 43: 10.220$ & 92.880 & -2.700 \\
\hline
\end{tabular}

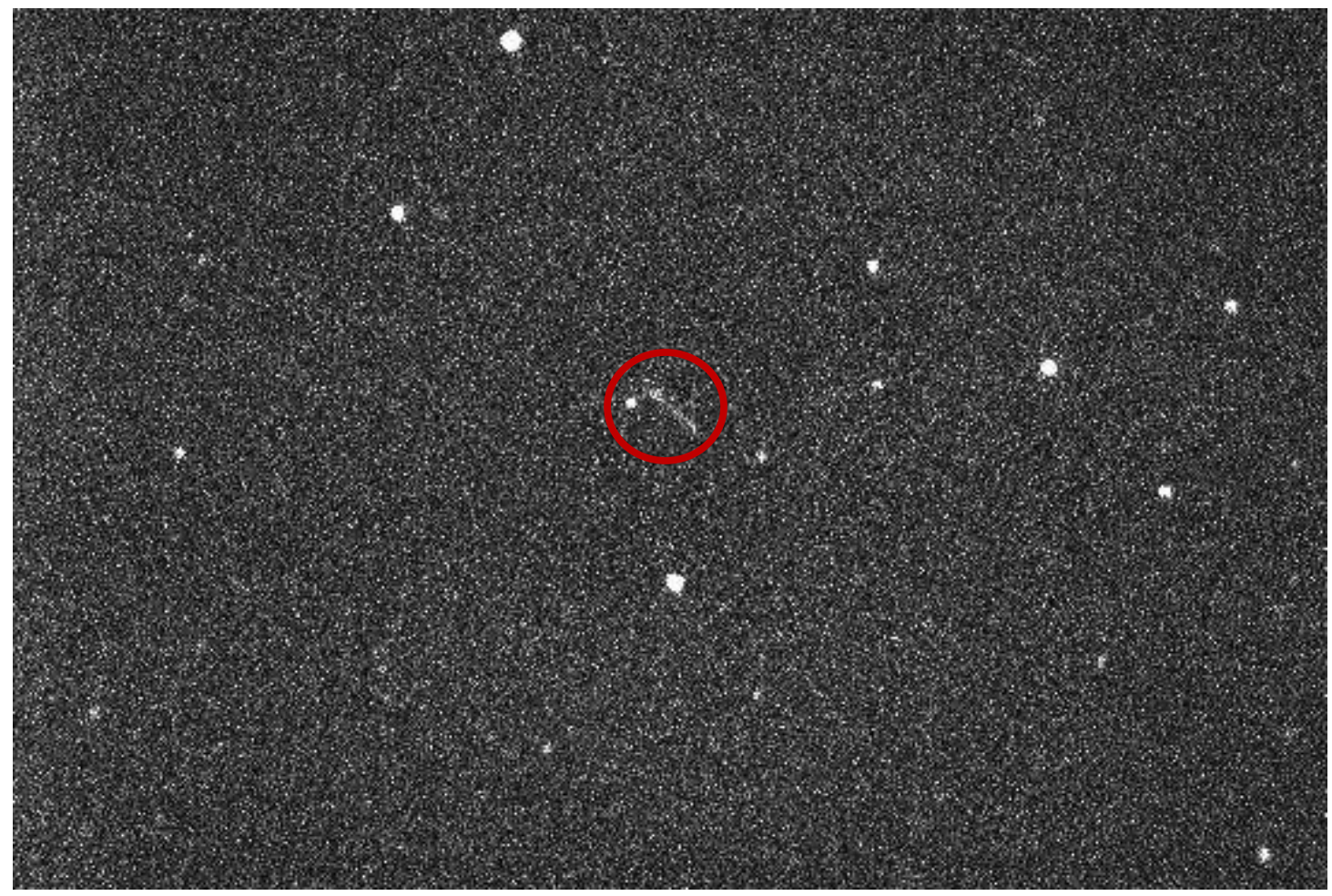

Figure A.2: Image taken of Globalstar M030, on March $15^{\text {th }}, 2016$, at 8:27 PM PST. This image represents the potentially dim signature of a passing satellite and can be caused by any number of reasons including solar phase angle and satellite orientation. 
Table A.2: Comparison star image pixel coordinates, right ascension, and declination for each usable satellite image

\begin{tabular}{|c|c|c|c|c|c|c|c|c|}
\hline \multirow{2}{*}{$\begin{array}{c}\text { Sat ID } \\
\text { Globalstar M026 }\end{array}$} & \multirow{2}{*}{$\frac{\text { StarX }}{205}$} & \multirow{2}{*}{$\begin{array}{c}\text { StarY } \\
315 \\
\end{array}$} & \multicolumn{3}{|c|}{ RA Star } & \multicolumn{3}{|c|}{ DEC Star } \\
\hline & & & 10 & 35 & 2.120 & 8 & 39 & 1.50 \\
\hline Globalstar M026 & 197 & 439 & 12 & 11 & 51.080 & 25 & 52 & 13.00 \\
\hline Globalstar M030 & 75 & 539 & 5 & 57 & 34.080 & -20 & -8 & -14.60 \\
\hline Globalstar M030 & 549 & 255 & 6 & 26 & 35.910 & -26 & -36 & -7.70 \\
\hline Globalstar M030 & 735 & 67 & 6 & 46 & 21.300 & -30 & -44 & -39.90 \\
\hline Globalstar M034 & 389 & 453 & 7 & 46 & 7.612 & 18 & 30 & 29.75 \\
\hline Globalstar M034 & 691 & 293 & 8 & 16 & 21.900 & 27 & 18 & 15.00 \\
\hline Globalstar M036 & 475 & 413 & 5 & 48 & 18.440 & 5 & 49 & 10.70 \\
\hline Globalstar M036 & 565 & 287 & 2 & 25 & 26.843 & 21 & 54 & 54.51 \\
\hline Globalstar M036 & 263 & 237 & 7 & 2 & 36.320 & 35 & 8 & 4.10 \\
\hline Globalstar M037 & 921 & 257 & 4 & 18 & 23.310 & 21 & 34 & 44.40 \\
\hline Globalstar M037 & 499 & 283 & 5 & 4 & 34.050 & 15 & 24 & 14.20 \\
\hline Globalstar M037 & 419 & 167 & 7 & 29 & 7.210 & -13 & -46 & -11.20 \\
\hline Globalstar M039 & 139 & 115 & 3 & 44 & 11.100 & 17 & 56 & 32.80 \\
\hline Globalstar M039 & 261 & 291 & 3 & 55 & 1.160 & 27 & 48 & 59.20 \\
\hline Globalstar M050 & 725 & 419 & 7 & 2 & 7.230 & 25 & 25 & 32.40 \\
\hline Globalstar M050 & 817 & 647 & 7 & 53 & 52.480 & 15 & 31 & 37.60 \\
\hline Globalstar M050 & 189 & 523 & 4 & 51 & 50.800 & 42 & 2 & 48.30 \\
\hline Globalstar M053 & 389 & 585 & 10 & 13 & 59.170 & -4 & -56 & -42.10 \\
\hline Globalstar M053 & 389 & 679 & 10 & 45 & 9.470 & 2 & 29 & 15.30 \\
\hline Globalstar M053 & 505 & 351 & 11 & 24 & 58.920 & 11 & 25 & 48.20 \\
\hline Globalstar M053 & 1033 & 407 & 12 & 0 & 38.070 & 18 & 20 & 5.80 \\
\hline Globalstar M053 & 445 & 387 & 12 & 32 & 38.140 & 23 & 15 & 39.60 \\
\hline Globalstar M053 & 883 & 185 & 13 & 15 & 36.430 & 28 & 44 & 29.30 \\
\hline Globalstar M053 & 203 & 583 & 14 & 2 & 11.870 & 32 & 29 & 33.60 \\
\hline Globalstar M056 & 845 & 345 & 4 & 37 & 15.080 & -15 & -29 & -47.80 \\
\hline Globalstar M056 & 685 & 361 & 5 & 7 & 2.670 & -6 & -30 & -55.60 \\
\hline Globalstar M056 & 693 & 291 & 5 & 33 & 20.860 & 1 & 51 & 11.60 \\
\hline Globalstar M056 & 267 & 269 & 6 & 0 & 34.470 & 10 & 6 & 11.30 \\
\hline Globalstar M056 & 847 & 233 & 6 & 28 & 3.620 & 18 & 11 & 55.90 \\
\hline Globalstar M058 & 85 & 207 & 5 & 7 & 38.380 & 9 & 28 & 13.70 \\
\hline Globalstar M058 & 665 & 29 & 5 & 46 & 24.140 & 31 & 16 & 25.10 \\
\hline Globalstar M062 & 527 & 375 & 5 & 48 & 2.240 & 11 & 36 & 33.00 \\
\hline Globalstar M062 & 659 & 57 & 8 & 4 & 56.890 & -19 & -12 & -9.50 \\
\hline Globalstar M069 & 949 & 271 & 4 & 24 & 37.570 & 33 & 57 & 33.10 \\
\hline Globalstar M069 & 285 & 213 & 5 & 23 & 22.910 & 28 & 56 & 15.30 \\
\hline Globalstar M069 & 339 & 315 & 6 & 20 & 32.130 & 21 & 5 & 26.80 \\
\hline Globalstar M069 & 801 & 491 & 6 & 58 & 11.810 & 14 & 13 & 13.20 \\
\hline Globalstar M069 & 757 & 13 & 4 & 30 & 37.450 & 13 & 43 & 27.40 \\
\hline
\end{tabular}

\section{Continued...}




\begin{tabular}{|c|c|c|c|c|c|c|c|c|}
\hline Sat ID & StarX & StarY & \multicolumn{3}{|c|}{ RA Star } & \multicolumn{3}{c|}{ DEC Star } \\
\hline Globalstar M069 & 391 & 367 & 5 & 4 & 41.976 & 7 & 45 & 57.95 \\
\hline Globalstar M081 & 795 & 215 & 5 & 32 & 12.790 & 0 & 47 & 39.40 \\
\hline Globalstar M081 & 441 & 261 & 6 & 13 & 13.790 & 12 & 39 & 18.10 \\
\hline Globalstar M089 & 853 & 127 & 7 & 7 & 18.440 & 4 & 40 & 0.50 \\
\hline Globalstar M089 & 781 & 277 & 8 & 30 & 25.300 & -13 & -28 & -7.30 \\
\hline Globalstar M094 & 559 & 293 & 4 & 54 & 43.740 & 12 & 21 & 5.50 \\
\hline Globalstar M094 & 753 & 495 & 5 & 32 & 56.130 & 5 & 18 & 40.70 \\
\hline Globalstar M094 & 335 & 557 & 6 & 10 & 51.485 & -2 & -36 & -14.77 \\
\hline
\end{tabular}

Table A.3: Usable satellite image pixel coordinates, RA and DEC

\begin{tabular}{|c|c|c|c|c|c|c|c|c|}
\hline Sat ID & SC1X & SC1Y & \multicolumn{2}{l|}{ RA.SC1 } & \multicolumn{2}{l|}{ DEC.SC1 } \\
\hline Globalstar M026 & 515 & 625 & 10 & 35 & 31.764 & 8 & 46 & 27.546 \\
\hline Globalstar M026 & 445 & 567 & 12 & 12 & 17.315 & 25 & 55 & 16.297 \\
\hline Globalstar M030 & 789 & 97 & 5 & 58 & 43.348 & -20 & -3 & -21.163 \\
\hline Globalstar M030 & 527 & 417 & 6 & 26 & 33.296 & -25 & -20 & -1.466 \\
\hline Globalstar M030 & 521 & 443 & 6 & 46 & 1.462 & -29 & -5 & -59.266 \\
\hline Globalstar M034 & 341 & 399 & 7 & 46 & 2.327 & 18 & 29 & 19.046 \\
\hline Globalstar M034 & 325 & 635 & 8 & 15 & 47.205 & 27 & 27 & 21.036 \\
\hline Globalstar M036 & 567 & 441 & 5 & 48 & 27.466 & 5 & 49 & 37.847 \\
\hline Globalstar M036 & 481 & 503 & 2 & 25 & 20.363 & 22 & 0 & 13.049 \\
\hline Globalstar M036 & 479 & 545 & 7 & 3 & 4.738 & 35 & 14 & 53.105 \\
\hline Globalstar M037 & 711 & 321 & 4 & 18 & 3.083 & 21 & 37 & 1.275 \\
\hline Globalstar M037 & 203 & 569 & 5 & 4 & 9.780 & 15 & 32 & 5.897 \\
\hline Globalstar M037 & 627 & 679 & 7 & 29 & 33.029 & -12 & -2 & -16.006 \\
\hline Globalstar M039 & 1083 & 433 & 3 & 45 & 45.614 & 18 & 4 & 6.350 \\
\hline Globalstar M039 & 951 & 81 & 3 & 56 & 13.966 & 27 & 43 & 0.641 \\
\hline Globalstar M050 & 425 & 573 & 7 & 1 & 37.968 & 25 & 30 & 6.080 \\
\hline Globalstar M050 & 389 & 735 & 7 & 53 & 12.469 & 15 & 35 & 32.458 \\
\hline Globalstar M050 & 885 & 69 & 4 & 53 & 25.293 & 41 & 53 & 39.366 \\
\hline Globalstar M053 & 727 & 683 & 10 & 14 & 30.925 & -3 & 0 & -34.950 \\
\hline Globalstar M053 & 587 & 467 & 10 & 45 & 26.972 & 2 & 23 & 54.475 \\
\hline Globalstar M053 & 651 & 529 & 11 & 25 & 12.851 & 11 & 30 & 5.551 \\
\hline Globalstar M053 & 619 & 549 & 11 & 59 & 57.156 & 18 & 23 & 52.675 \\
\hline Globalstar M053 & 567 & 489 & 12 & 32 & 50.667 & 23 & 18 & 7.295 \\
\hline Globalstar M053 & 485 & 515 & 13 & 14 & 51.689 & 28 & 51 & 55.575 \\
\hline Globalstar M053 & 397 & 585 & 14 & 2 & 33.772 & 32 & 29 & 39.207 \\
\hline Globalstar M056 & 621 & 549 & 4 & 36 & 52.371 & -14 & -25 & -31.945 \\
\hline Globalstar M056 & 693 & 427 & 5 & 7 & 3.488 & -5 & -27 & -30.217 \\
\hline Globalstar M056 & 707 & 435 & 5 & 33 & 22.709 & 1 & 54 & 36.410 \\
\hline
\end{tabular}

Continued... 


\begin{tabular}{|l|c|c|c|c|c|c|c|c|}
\hline Globalstar M056 & 891 & 643 & 6 & 1 & 35.941 & 10 & 14 & 37.307 \\
\hline Globalstar M056 & 817 & 281 & 6 & 28 & 1.315 & 18 & 13 & 9.783 \\
\hline Globalstar M058 & 749 & 689 & 5 & 8 & 48.645 & 9 & 37 & 15.064 \\
\hline Globalstar M058 & 761 & 463 & 5 & 46 & 33.016 & 31 & 26 & 49.781 \\
\hline Globalstar M062 & 629 & 541 & 5 & 48 & 13.245 & 11 & 40 & 19.529 \\
\hline Globalstar M062 & 463 & 735 & 8 & 4 & 41.622 & -18 & -31 & -25.922 \\
\hline Globalstar M069 & 897 & 67 & 4 & 24 & 29.233 & 33 & 52 & 50.836 \\
\hline Globalstar M069 & 575 & 455 & 5 & 23 & 57.366 & 29 & 1 & 9.512 \\
\hline Globalstar M069 & 215 & 735 & 6 & 20 & 24.704 & 21 & 15 & 43.673 \\
\hline Globalstar M069 & 367 & 735 & 6 & 57 & 32.956 & 14 & 20 & 25.242 \\
\hline Globalstar M069 & 545 & 487 & 4 & 30 & 22.879 & 13 & 55 & 18.096 \\
\hline Globalstar M069 & 529 & 463 & 5 & 4 & 55.495 & 7 & 48 & 9.542 \\
\hline Globalstar M081 & 591 & 627 & 5 & 31 & 54.175 & 0 & 57 & 33.829 \\
\hline Globalstar M081 & 617 & 429 & 6 & 13 & 31.497 & 12 & 43 & 9.732 \\
\hline Globalstar M089 & 897 & 395 & 7 & 7 & 22.200 & 4 & 46 & 24.373 \\
\hline Globalstar M089 & 849 & 647 & 8 & 30 & 32.815 & -12 & -23 & -6.682 \\
\hline Globalstar M094 & 687 & 397 & 4 & 54 & 57.234 & 12 & 23 & 13.684 \\
\hline Globalstar M094 & 617 & 571 & 5 & 32 & 43.546 & 5 & 20 & 39.777 \\
\hline Globalstar M094 & 519 & 735 & 6 & 11 & 9.975 & -1 & -19 & -46.701 \\
\hline
\end{tabular}




\section{Appendix B LESSONS LEARNED}

\section{Anticipate Bad Weather and Available Observation Slots}

The main cause in delay of progress for an observation based project is undoubtedly the weather. It is wise to know the forecast for the days and/or weeks leading to proposed observation nights. It turns out that although an intended satellite target may be in perfect viewing position, the weather conditions will not always agree. An awareness of other observers using the same instrumentation and being mindful of other ongoing projects alongside your own to reduce conflict in observation times. It is far easier to work with the other observers and setting observation dates that align with your target's optimal position, clear skies, and non-conflicting usage of the equipment.

\section{Using Optimal Available Equipment for Goal}

When first setting up your thesis goals and procedures, it is important to know what equipment is available to you and to take advantage of it. The overall thesis goals and the desired image qualities conducive to your topic will dictate the equipment that 
should be used. An example of this is that instead of using the $f / 6.3$ focal reducer, it may have yielded a better image if an $\mathrm{f} / 3.3$ focal reducer was implemented in order to increase the FOV of the telescope and thus giving me a better chance of imaging a passing satellite. The reduced apparent focal length would also increase the brightness of the targets within the images. This would seemingly allow for fainter objects to be visible and could prove to be useful when observing nanosatellites in the future.

\section{Orbital Elevation Timing and Solar Phase Angles of Nanosatellites}

The orbital positioning of a satellite relative to the observation location is extremely important when attempting to observe such a small object. It is important to not only determine the RAAN of the satellite but also the SPA. Even if a satellite is at a high enough elevation to view, it may not be visible for the majority of the pass due to the relative location to the Sun and the observer.

\section{Calibration of Telescope Pointing and Computer Clock}

Although the telescope was calibrated at the beginning of each night of observation, it was soon realized that the telescope pointing accuracy changed drastically when slewing from one observation point to another which is even more prevalent when slewing large distances. It is necessary to recalibrate the telescopes pointing for each location after slewing to ensure the most accurate pointing accuracy. 


\section{Fit Gaussian Curve to Stars}

The use of a Gaussian fit within the IRAF software would prove to be a useful tool in pinpointing the central point of comparison stars within the scientific images as well as the central points of the satellite end points. The increased accuracy in determining the central pixel based on the analysis of the Gaussian distribution of photons would decrease the error within the pixel selection process. 


\section{Appendix

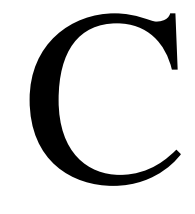

\section{SATELLITE TARGET ORBITAL DATA}

This section contains the remaining orbital determination data previously

referenced in the thesis. Some data sets were unable to converge due to errors within the time stamps and omitted from the final data tables.

Table C.1: COE comparison of observed satellites between the initial TLE, the two Gauss methods, and the double-r method

\begin{tabular}{|c|c|c|c|c|c|c|c|}
\hline Satellite ID & Source & Epoch (UTC) & $a(\mathrm{~km})$ & e & $i\left({ }^{\circ}\right)$ & $\Omega\left({ }^{\circ}\right)$ & $\omega\left(^{\circ}\right)$ \\
\hline \multirow{4}{*}{$\begin{array}{c}\text { Globalstar } \\
\text { M030 }\end{array}$} & TLE & $3 / 1519: 24$ & 8270.5 & $9.310 \mathrm{E}-04$ & 51.917 & 296.001 & 102.900 \\
\hline & Vallado Gauss & \multirow{3}{*}{$3 / 163: 27$} & 9056.0 & $9.631 \mathrm{E}-02$ & 48.446 & 295.681 & 210.673 \\
\hline & Curtis Gauss & & 9044.4 & $9.629 \mathrm{E}-02$ & 48.439 & 295.685 & 210.738 \\
\hline & Double-r & & 9044.4 & $9.629 \mathrm{E}-02$ & 48.439 & 295.685 & 210.739 \\
\hline \multirow{4}{*}{$\begin{array}{c}\text { Globalstar } \\
\text { M037 }\end{array}$} & TLE & 4/5 3:26 & 7788.7 & $6.370 \mathrm{E}-04$ & 51.956 & 334.714 & 51.590 \\
\hline & Vallado Gauss & \multirow{3}{*}{$4 / 64: 19$} & 7760.7 & $2.350 \mathrm{E}-03$ & 51.392 & 332.275 & 262.706 \\
\hline & Curtis Gauss & & 7753.2 & $2.291 \mathrm{E}-03$ & 51.449 & 332.322 & 264.206 \\
\hline & Double-r & & 7753.2 & $2.291 \mathrm{E}-03$ & 51.449 & 332.322 & 264.214 \\
\hline \multirow{4}{*}{$\begin{array}{c}\text { Globalstar } \\
\text { M050 }\end{array}$} & TLE & $4 / 53: 26$ & 8027.1 & $1.457 \mathrm{E}-03$ & 51.973 & 339.028 & 128.602 \\
\hline & Vallado Gauss & \multirow{3}{*}{$4 / 63: 48$} & 8812.5 & $3.622 \mathrm{E}-02$ & 49.629 & 337.113 & 314.727 \\
\hline & Curtis Gauss & & 8820.8 & $3.845 \mathrm{E}-02$ & 49.311 & 334.087 & 314.998 \\
\hline & Double-r & & 8820.8 & $3.845 \mathrm{E}-02$ & 49.311 & 334.087 & 314.998 \\
\hline \multirow{4}{*}{$\begin{array}{c}\text { Globalstar } \\
\text { M053 }\end{array}$} & TLE & $4 / 114: 50$ & 8401.0 & $7.880 \mathrm{E}-04$ & 52.070 & 124.403 & 100.680 \\
\hline & Vallado Gauss & \multirow{3}{*}{$4 / 24: 41$} & 7793.1 & $7.164 \mathrm{E}-02$ & 52.159 & 122.877 & 220.288 \\
\hline & Curtis Gauss & & 7769.0 & $7.254 \mathrm{E}-02$ & 52.222 & 122.838 & 219.566 \\
\hline & Double-r & & 7769.0 & $7.254 \mathrm{E}-02$ & 52.222 & 122.838 & 219.566 \\
\hline \multirow{4}{*}{$\begin{array}{c}\text { Globalstar } \\
\text { M056 }\end{array}$} & TLE & $3 / 164: 12$ & 8039.3 & $9.110 \mathrm{E}-04$ & 52.087 & 78.944 & 84.489 \\
\hline & $\begin{array}{l}\text { Vallado Gauss } \\
\end{array}$ & \multirow{3}{*}{ 3/17 3:32 } & 7122.6 & $6.727 \mathrm{E}-02$ & 51.342 & 74.499 & 218.135 \\
\hline & Curtis Gauss & & 7076.5 & $6.724 \mathrm{E}-02$ & 51.345 & 74.505 & 218.114 \\
\hline & Double-r & & 7076.5 & $6.724 \mathrm{E}-02$ & 51.345 & 74.505 & 218.114 \\
\hline \multirow{4}{*}{$\begin{array}{c}\text { Globalstar } \\
\text { M069 }\end{array}$} & TLE & $4 / 43: 56$ & 7790.9 & $7.120 \mathrm{E}-04$ & 51.958 & 335.137 & 98.175 \\
\hline & Vallado Gauss & \multirow{3}{*}{ 4/5 3:42 } & 8283.7 & $5.679 \mathrm{E}-02$ & 52.259 & 334.627 & 314.542 \\
\hline & Curtis Gauss & & 8226.3 & $5.627 \mathrm{E}-02$ & 52.262 & 334.625 & 314.544 \\
\hline & Double-r & & 8226.3 & $5.627 \mathrm{E}-02$ & 52.262 & 334.625 & 314.544 \\
\hline
\end{tabular}


Table C.2: CTE and ATE values for observed satellites along with the target and observed RA and DEC. Target RA and DEC provided by STK software

\begin{tabular}{|c|c|c|c|c|c|c|c|c|}
\hline Sat ID & Target RA $\left({ }^{\circ}\right)$ & Det. RA $\left({ }^{\circ}\right)$ & $\Delta R A\left({ }^{\circ}\right)$ & Target DEC $\left({ }^{\circ}\right)$ & Det. DEC $\left({ }^{\circ}\right)$ & $\triangle \mathrm{DEC}\left({ }^{\circ}\right)$ & CTE (km) & ATE $(\mathrm{km})$ \\
\hline GS M026 & 158.88539 & 158.86360 & $2.18 \mathrm{E}-02$ & 8.46537 & 8.75550 & $2.90 \mathrm{E}-01$ & 0.0467 & 0.0102 \\
\hline GS M026 & 183.17446 & 183.05540 & 1.19E-01 & 25.78285 & 25.91330 & $1.30 \mathrm{E}-01$ & 0.0525 & 0.0210 \\
\hline GS M030 & 89.60849 & 89.63740 & $2.89 \mathrm{E}-02$ & -20.18591 & -20.30220 & $1.16 \mathrm{E}-01$ & 10.2089 & 0.0131 \\
\hline GS M030 & 96.76323 & 96.63990 & $1.23 \mathrm{E}-01$ & -26.62944 & -26.54790 & $8.15 \mathrm{E}-02$ & 50.5813 & 0.0159 \\
\hline GS M030 & 101.77184 & 101.51680 & $2.55 \mathrm{E}-01$ & -30.86332 & -30.61230 & $2.51 \mathrm{E}-01$ & 61.0061 & 0.0192 \\
\hline GS M034 & 116.78565 & 116.51260 & $2.73 \mathrm{E}-01$ & 18.48398 & 18.49130 & 7.32E-03 & 0.0251 & 0.0120 \\
\hline GS M034 & 124.49540 & 123.96780 & $5.28 \mathrm{E}-01$ & 27.14740 & 27.43280 & $2.85 \mathrm{E}-01$ & 0.9832 & 0.0140 \\
\hline GS M036 & 87.32625 & 87.10850 & $2.18 \mathrm{E}-01$ & 5.76384 & 5.82580 & $6.20 \mathrm{E}-02$ & 0.1930 & 0.0240 \\
\hline GS M036 & 96.60439 & 96.33850 & $2.66 \mathrm{E}-01$ & 21.78508 & 21.99010 & 2.05E-01 & 0.3395 & 0.0111 \\
\hline GS M036 & 105.75577 & 105.75150 & $4.27 \mathrm{E}-03$ & 34.93953 & 35.23090 & 2.91E-01 & 0.1929 & 0.0129 \\
\hline GS M037 & 64.90181 & 64.52500 & 3.77E-01 & 21.58692 & 21.61100 & $2.41 \mathrm{E}-02$ & 0.0027 & 0.0141 \\
\hline GS M037 & 76.45767 & 76.05550 & $4.02 \mathrm{E}-01$ & 15.31520 & 15.51520 & 2.00E-01 & 0.3197 & 0.0140 \\
\hline GS M037 & 112.30010 & 112.37160 & $7.15 \mathrm{E}-02$ & -13.91988 & -13.60630 & 3.14E-01 & 43.2075 & 0.0100 \\
\hline GS M039 & 55.94309 & 56.38080 & $4.38 \mathrm{E}-01$ & 17.92854 & 18.04940 & $1.21 \mathrm{E}-01$ & 2.0446 & 0.0124 \\
\hline GS M039 & 58.99372 & 59.01240 & $1.87 \mathrm{E}-02$ & 27.84454 & 27.73130 & $1.13 \mathrm{E}-01$ & 1.6782 & 0.0174 \\
\hline GS M050 & 105.90236 & 105.42600 & 4.76E-01 & 25.32385 & 25.49010 & $1.66 \mathrm{E}-01$ & 0.0495 & 0.0146 \\
\hline GS M050 & 118.83123 & 118.32650 & 5.05E-01 & 15.44730 & 15.58230 & $1.35 \mathrm{E}-01$ & 0.2883 & 0.0241 \\
\hline GS M050 & 73.14183 & 73.29620 & $1.54 \mathrm{E}-01$ & 41.99340 & 41.91680 & 7.66E-02 & 0.8354 & 0.0086 \\
\hline GS M053 & 153.59142 & 153.60900 & $1.76 \mathrm{E}-02$ & -5.06073 & -4.90690 & $1.54 \mathrm{E}-01$ & 56.4356 & 0.0226 \\
\hline GS M053 & 161.40043 & 161.35110 & 4.93E-02 & 2.44162 & 2.41150 & $3.01 \mathrm{E}-02$ & 0.7250 & 0.0175 \\
\hline GS M053 & 171.43180 & 171.29460 & 1.37E-01 & 11.29348 & 11.49050 & 1.97E-01 & 0.0996 & 0.0213 \\
\hline GS M053 & 188.35817 & 188.20300 & $1.55 \mathrm{E}-01$ & 23.15474 & 23.29560 & $1.41 \mathrm{E}-01$ & 0.0447 & 0.0214 \\
\hline GS M053 & 210.70084 & 210.62670 & 7.41E-02 & 32.42395 & 32.49370 & $6.98 \mathrm{E}-02$ & 0.1979 & 0.0107 \\
\hline GS M056 & 83.56312 & 83.34310 & $2.20 \mathrm{E}-01$ & 1.83488 & 1.90130 & $6.64 \mathrm{E}-02$ & 0.1486 & 0.0212 \\
\hline GS M056 & 90.16850 & 90.36110 & $1.93 \mathrm{E}-01$ & 10.00828 & 10.22240 & $2.14 \mathrm{E}-01$ & 0.0568 & 0.0133 \\
\hline GS M056 & 97.33471 & 97.00650 & $3.28 \mathrm{E}-01$ & 18.23550 & 18.21600 & $1.95 \mathrm{E}-02$ & 0.0941 & 0.0219 \\
\hline GS M058 & 76.89330 & 77.15860 & $2.65 \mathrm{E}-01$ & 9.32100 & 9.59800 & 2.77E-01 & 1.1705 & 0.0155 \\
\hline GS M058 & 86.82198 & 86.63150 & $1.90 \mathrm{E}-01$ & 31.11976 & 31.42090 & 3.01E-01 & 0.1686 & 0.0143 \\
\hline GS M062 & 87.25894 & 87.04800 & 2.11E-01 & 11.47346 & 11.66240 & 1.89E-01 & 0.3606 & 0.0095 \\
\hline GS M062 & 121.18013 & 121.18230 & 2.17E-03 & -19.22585 & -18.97040 & $2.55 \mathrm{E}-01$ & 11.9664 & 0.0101 \\
\hline GS M069 & 66.51158 & 66.12660 & $3.85 \mathrm{E}-01$ & 34.04648 & 33.89220 & $1.54 \mathrm{E}-01$ & 0.4258 & 0.0194 \\
\hline GS M069 & 80.91344 & 80.96720 & $5.38 \mathrm{E}-02$ & 28.90326 & 29.00670 & $1.03 \mathrm{E}-01$ & 0.6506 & 0.0147 \\
\hline GS M069 & 95.38322 & 95.10720 & $2.76 \mathrm{E}-01$ & 20.94178 & 21.23620 & 2.94E-01 & 0.5232 & 0.0142 \\
\hline GS M069 & 104.96417 & 104.41110 & $5.53 \mathrm{E}-01$ & 14.05689 & 14.32210 & 2.65E-01 & 0.0439 & 0.0195 \\
\hline GS M069 & 67.92089 & 67.60410 & 3.17E-01 & 13.59081 & 13.89190 & 3.01E-01 & 0.9620 & 0.0128 \\
\hline GS M069 & 76.32749 & 76.22250 & $1.05 \mathrm{E}-01$ & 7.76415 & 7.79680 & $3.26 \mathrm{E}-02$ & 0.4289 & 0.0171 \\
\hline GS M081 & 83.34311 & 82.98690 & $3.56 \mathrm{E}-01$ & 0.66846 & 0.93440 & $2.66 \mathrm{E}-01$ & 0.7395 & 0.0239 \\
\hline GS M081 & 93.49791 & 93.36990 & $1.28 \mathrm{E}-01$ & 12.61565 & 12.70940 & 9.37E-02 & 0.0594 & 0.0128 \\
\hline GS M089 & 107.06764 & 106.83980 & $2.28 \mathrm{E}-01$ & 4.50993 & 4.75720 & 2.47E-01 & 0.4685 & 0.0106 \\
\hline GS M089 & 127.80480 & 127.63180 & $1.73 \mathrm{E}-01$ & -13.68619 & -13.34470 & $3.41 \mathrm{E}-01$ & 24.4028 & 0.0207 \\
\hline GS M094 & 73.84117 & 73.72970 & $1.11 \mathrm{E}-01$ & 12.35374 & 12.38150 & $2.78 \mathrm{E}-02$ & 0.4386 & 0.0146 \\
\hline GS M094 & 83.52646 & 83.18890 & $3.38 \mathrm{E}-01$ & 5.25729 & 5.33910 & $8.18 \mathrm{E}-02$ & 0.0044 & 0.0178 \\
\hline GS M094 & 92.88008 & 92.77970 & $1.00 \mathrm{E}-01$ & -2.69979 & -2.54810 & $1.52 \mathrm{E}-01$ & 36.8188 & 0.0111 \\
\hline
\end{tabular}




\section{Appendix D \\ OBSERVATION PROCEDURE}

\section{Preparation Stage:}

- Download most recent desired target's TLEs from SpaceTrack.org (or other source) and place into MATLAB directory in .txt file format.

- Use passPredict.m to check for visible passes for specified observation time. Read the FUNCTION DESCRIPTION section to see how to use the function-included is an example command to test the function. The user will also be queried for additional information at different points in the function. [23]

- Use STK to generate azimuth/elevation angles, access times, and illumination times for satellites found to be visible by passPredict.m:

- $\quad$ Insert $\rightarrow$ New $\ldots \rightarrow$ Satellite $\rightarrow$ From Standard Object Database $\rightarrow$ Enter SSC Number (Column 1 from passPredict output) $\rightarrow$ Right Click "Satellite" $\rightarrow$ Properties $\rightarrow$ Set Time Interval \& Add TLEs from either AGI, SpaceTrack server, or import from .txt. file containing single TLE

- Insert $\rightarrow$ New $\ldots \rightarrow$ Facility (Properties) $\rightarrow$ Position $\rightarrow$ Set observatory's latitude/longitude/altitude

- Insert $\rightarrow$ New ... $\rightarrow$ Sensor (Properties) $\rightarrow$ Definition $\rightarrow$ Set sensor type to "Simple Conic" with $70^{\circ}$ Half Cone Angle

- Right Click Satellite $\rightarrow$ Report and Graph Manager $\rightarrow$ Specify Time Period $\rightarrow$ Installed Styles $\rightarrow$ Lighting Times $\rightarrow$ Generate

- Right Click "Sensor" $\rightarrow$ Access $\rightarrow$ Select Satellite $\rightarrow$ Specify Time Period (U.T) $\rightarrow$ Reports: AER $\rightarrow$ Save as .csv file 
- Take data to Observatory Terminal and boot up Telescope Systems and SkyX software

Observatory Set-Up Stage: (Provided by the Cal Poly Physics Department)

- Turn on dome lights (white and red)

- Turn on power strip on wall next to dome controls

- Open computer software:

- Turn on computer (computer box is in cabinet on the left side of the desk)

- Login (Ask Physics department for terminal login information)

- Start telescope control software from desktop (TheSkyX Professional Edition)

- Under Telescope/Telescope Setup tab, select: Dome, Dome Setup, Connect

- Open dome:

- Under left menu, select: Dome, Open Dome

- If low altitude observations are needed, attach lower windbreak to slit with chain before opening slit, but only do that if really necessary

- Open telescope:

- Using the ladder, perform the following, being very careful to not move the telescope!

- Remove tarp cover from telescope (put it outside the dome)

- Remove lens caps from telescope, finder, and StarLock (4 total; put in safe place)

- Turn on telescope power with switch at base

- Wait until it finds a GPS signal; the handpaddle should say "select item"

- Make sure that StarLock is turned off. To do so, take the handpaddle, and use the bottom arrow keys to navigate in the manual to Utilities. Hit enter. Navigate to StarLock. Hit enter. Navigate to On/Off. Hit Enter. Navigate to Off. Hit enter. Press mode until you are back in "Select Item: Object".

- Begin computer control of electronics:

- Under Telescope/Telescope Setup tab, select: Mount, Mount Setup, Connect 
- Turn on CCD camera power (switch on power adaptor underneath shelf behind telescope)

- Turn on focus controller (on the desk between monitors; note that it should be set to "manual" mode)

- Under Telescope/Telescope Setup tab, select: Camera, Camera Setup, Connect. The Camera window will appear as a separate window on the right screen. If it does not, in TheSkyX, go to Display and select Camera from the options in the pull-down menu below.

- In Camera window, select Temp. Setup, begin temperature control of $\mathrm{CCD}$ (should cool to $-5^{\circ} \mathrm{C}$; Fan on)

- Under Telescope/Telescope Setup tab, select: Filter Wheel, Filter Wheel Setup, Connect (this might connect automatically with the camera)

- Under Telescope/Telescope Setup tab, select: Focuser, Focuser Setup, Connect (note that this will only work if the focuser has been on for a little while and moved to its last position, so be patient)

- Prepare for data taking:

- Create a folder on the Desktop with the name of your group observing $\log$ file and log book

- Fill in log book (see example; always keep it on desk next to monitors)

- Fill in log files as you take exposures (see example; more empty logfiles are on the desk; help yourself as need be; you can also find them on PolyLearn)

- Assign a person to be the scribe while another person takes exposures

- Take sky flats:

- Using TheSkyX, move the telescope to the optimal location for flats $\left(20^{\circ}\right.$ from zenith, opposite Sun direction; that corresponds to an altitude of $70^{\circ}$ and an azimuth of $90^{\circ}$ ). Do so by clicking with the mouse on that location and tell the telescope to slew to your mouse position. The dome should automatically track the telescope.

- Switch off dome lights (white)

- In Camera window, select Exposure time: 1.000 seconds, Exposure delay: 0.00 seconds, Binning 1x1, Frame: Flat Field, Reduction: None, Automatically save photo to the folder with your name, create a datebased subfolder

- Set the sequence number to always start with 1000

- Check that the filter is the one you will be using for your science exposures

- Take $\sim 1 \mathrm{sec}$ exposure of the sky, so the chip is not saturated (30-40k counts is best) 
- Once you take the first exposure, it will open another window next to the camera, displaying the image. You can zoom in and out, change the histogram, check the count level of your exposure or check the exposure for structure (for example, when you move with the cursor over the image, it will show "( $\mathrm{x}, \mathrm{y})$ count level" to the lower left)

- You might have to wait until the sky is only moderately bright.

- Continue to take flats, increasing exposure time as necessary

- Between each flat field, dither the telescope by a small amount (Move, Rate "center")

- 8 flats are sufficient, more is better

- Double check that your photos are actually saved!

- Take biases:

- Bias exposures have no exposure time.

- You might want to cover the mirror to reduce any scattered light.

- In Camera window, select Exposure time: 0.000 seconds, Exposure delay: 0.00 seconds, Binning 1x1, Frame: Bias, Reduction: None, Automatically save photos

- Take one bias and double check. A bias should have no structure (except for bad pixels) with counts between 90-130.

- If it looks good, take 11 more bias exposures; you can use the "Take Series" option of the Camera control for this purpose

- Take darks (optional):

- Dark exposures should have the same exposure time as the science exposures

- In Camera window, select Exposure time: like your science exposures, Exposure delay: seconds, Binning 1x1, Frame: Dark, Reduction: None, Automatically save photos

- $\quad$ Take 8-12 dark exposures for each exposure time you will use

- This can be done at the end of the night as well

- Fix telescope pointing:

- Find a known bright star, click on that star with TheSkyX, slew to that star

- In Camera window, select Exposure time: 0.100 seconds, Exposure delay: 0.00 seconds, Binning 1x1, Frame: Light, Reduction: None, Automatically save photos

- Take image of star

- If the star is not on the CCD, use the finder scope to center star on crosshairs

- Do not use the handpaddle to center the star, use TheSkyX 
- Under left menu, select Telescope, Move, Rate: Move or Center and the arrow keys (that means that one person looks through the finder, the other slowly moves the telescope).

- Once the star is in the center of the finder scope, take another image

- Move the telescope as needed to center star on CCD (Rate: Center; one click at a time)

- You can switch on the crosshair display on the image, to help you determine the center of the $\mathrm{CCD}$

- Note that the arrow keys move the telescope; so if you want the star to move left on the CCD, you will have to move the telescope to the right and so on.

- Sync telescope to that star with TheSkyX

- Focus telescope:

- Slew to a bright star, but not brighter than 4 mag, for exposure times $0.1 \mathrm{sec}$ or longer

- Important: the focus will depend strongly on the filter you are using; see offsets below!

- Take image of star to determine good exposure time (not saturated, but with an obviously bright star)

- In Camera window, select Focus Tools, Graphs

- Set exposure time depending on the brightness of your star, so that it is not saturated but you get enough counts; exposure delay: $3 \mathrm{sec}$; take photo continuously; take photo

- Under left menu, select Focuser, Move in or Move out by 50

- During the exposure delay, move the focus in one direction in small steps (e.g. 50)

- Do this for a while, then go into other direction for a while, until you see true minimum

- Monitor the graphs, the most interesting one is the lowest, you can deselect the rest

- You want to find the smallest Half Flux Diameter (HFD) and note this focuser position

- Set it to this focuser position and note it in your log file as well as the observatory log book

\section{Observation Stage:}

- Upload TLE .txt file to terminal

- Open TheSkyX software

- Click on the "input" tab

- Click on the "Satellites..." tab

- Make sure "Show satellite's path" is turned on 
- Click on "Import from file"

- Choose desired TLE file

- Click on "Satellite from above Earth"

- Highlight satellite orbit wanted

- Click on "Show selected orbit"

- Click on "Find best passes"

- Choose the most current path

- Dither telescope to RA and DEC of first time stamp:

- Refocus on any star within the FOV and take image.

- Adjust the FOV until object is in center .

- Acquire and observe your targets:

- Reposition telescope back to center on RA and DEC of transit path

- Note: You will have to wait for the sky to be dark enough ( $\sim 1$ hour after sunset)

- In Camera window, select Exposure time: 0.2 seconds, Exposure delay: 0.00 seconds, Binning 1x1(or 2x2), Frame: Light, Reduction: None, Automatically save photos

- Wait for transit time of current RA and DEC on terminal internal clock and take snapshot:

- Snapshots should be saving directly to your predesignated file

- Note: It is extremely helpful to title each image prior to image being taken in the "Camera" window

- Dither telescope to each subsequent RA and DEC along transit path:

- If a large dither, the Telescopes pointing accuracy may deviate by a significant amount

- Refocus on any star in the FOV and adjust again to center

- Reposition telescope back to center on RA and DEC of transit path and take image

- Note: If dithering a small distance, re-centering may not be necessary

Observatory Closing Stage: (Provided by the Cal Poly Physics Department)

- After observing is complete:

- Take all your personal belongings and logsheets

- Switch off lights in E1 (but leave monitors as they are)

- Return to dome

- Switch on observatory monitors 
- Use the handpaddle and switch off StarLock (see instructions above).

- Turn on dome lights

- Park telescope:

- Use TheSkyX to park telescope (left menu, Meade, Park) (The handpaddle should say "Scope parked. Switch off scope.")

- Disconnect TheSkyX from the telescope (Telescope, Telescope Setup, Mount Setup, Disconnect)

- Immediately shut off telescope power (this stops the tracking)

- Replace all telescope mirror covers

- Cover telescope with black tarp

- Disconnect focuser, camera, filter wheel (under Telescope, Telescope Setup)

- Close dome:

- Under left menu, select Dome, Park Dome (this should close the dome and park the dome; electrical connection made opposite slit)

- If this fails for some reason, control the dome manually using the buttons next to the dome. (Note that the wires behind the glass screen need to line up to close dome.)

- Turn off equipment:

- Turn off power to focuser and camera

- Quit all computer programs, power down computer

- Turn off power strip on dome control panel

- Take all your personal belongings and logsheets

- Turn off dome lights

- Close dome door, make sure it is locked

- Lock gate 


\section{Appendix E}

\section{PIXEL ERROR ANALYSIS}

The effects of the pixel error uncertainties were analyzed through the MonteCarlo method. A series of 10,000 iterations with various minute alterations in multiple areas were performed for single-pass orbit determination, cross-track error, and alongtrack error. The astronomical uncertainties include pixel selection errors: \pm 1 pixel for comparison star positions, \pm 2 pixels for satellite positions, and $\pm 0.004 \mathrm{arcsec} / \mathrm{pix}$ for the plate scale. The reason for the smaller pixel variation for the comparison stars were due to the relatively small size compared to the satellite swath width. The variations in the timestamps as well as computer clock error were $\pm 1 \mathrm{~ms}$. Three single-trio observations for each satellite were analyzed generating $3 \sigma$ errors which can be found below in Table E.1. The positional errors equate to a distance of roughly 0.5 to 6 arc seconds of deviation which have a noticeably increased effect on the CTE values over the ATE values. The associated error values can be found in Tables 5.1 and 5.2. 
Table E.1: Uncertainties in the determined RA, DEC, CTE, and ATE values caused by error inherent to the pixel selection process

\begin{tabular}{|c|c|c|c|c|}
\hline Satellite ID & RA Error $^{\left({ }^{\circ}\right)}$ & DEC Error $^{\left({ }^{\circ}\right)}$ & CTE Error $(\mathbf{k m})^{\text {ATE Error }(\mathbf{k m})}$ \\
\hline \multirow{3}{*}{ Globalstar M053 } & $1.89 \mathrm{E}-02$ & $2.14 \mathrm{E}-02$ & 0.125 & 0.056 \\
& $1.12 \mathrm{E}-02$ & $2.42 \mathrm{E}-02$ & 0.576 & 0.077 \\
& $5.21 \mathrm{E}-03$ & $3.15 \mathrm{E}-03$ & 0.051 & 0.031 \\
\hline \multirow{3}{*}{ Globalstar M056 } & $2.35 \mathrm{E}-03$ & $4.72 \mathrm{E}-03$ & 0.094 & 0.012 \\
& $3.26 \mathrm{E}-03$ & $3.91 \mathrm{E}-03$ & 0.043 & 0.036 \\
& $1.99 \mathrm{E}-03$ & $2.12 \mathrm{E}-03$ & 0.067 & 0.009 \\
\hline \multirow{3}{*}{ Globalstar M069 } & $4.84 \mathrm{E}-03$ & $3.62 \mathrm{E}-03$ & 0.103 & 0.023 \\
& $3.11 \mathrm{E}-03$ & $2.89 \mathrm{E}-03$ & 0.076 & 0.042 \\
& $3.83 \mathrm{E}-03$ & $2.73 \mathrm{E}-03$ & 0.078 & 0.017 \\
\hline
\end{tabular}

